NBS SPECIAL PUBLICATION 400-2

Semiconductor Measurement Technology:

U.S. RTMENT OF IMERCE National Bureau of tandards 


\section{NATIONAL BUREAU OF STANDARDS}

The National Bureau of Standards ' was established by an act of Congress March 3, 1901. The Bureau's overall goal is to strengthen and advance the Nation's science and technology and facilitate their effective application for public benefit. To this end, the Bureau conducts research and provides: (1) a basis for the Nation's physical measurement system, (2) scientific and technological services for industry and government, (3) a technical basis for equity in trade, and (4) technical services to promote public safety. The Bureau consists of the Institute for Basic Standards, the Institute for Materials Research, the Institute for Applied Technology, the Institute for Computer Sciences and Technology, and the Office for Information Programs.

THE INSTITUTE FOR BASIC STANDARDS provides the central basis within the United States of a complete and consistent system of physical measurement; coordinates that system with measurement systems of other nations; and furnishes essential services leading to accurate and uniform physical measurements throughout the Nation's scientific community, industry, and commerce. The Institute consists of a Center for Radiation Research, an Office of Measurement Services and the following divisions:

Applied Mathematics - Electricity - Mechanics - Heat - Optical Physics - Nuclear Sciences ${ }^{2}$ - Applied Radiation ${ }^{2}$ - Quantum Electronics ${ }^{3}$ - Electromagnetics ${ }^{3}$ - Time and Frequency ${ }^{3}$ - Laboratory Astrophysics ${ }^{3}$ - Cryogenics ${ }^{3}$.

THE INSTITUTE FOR MATERIALS RESEARCH conducts materials research leading to improved methods of measurement, standards, and data on the properties of well-characterized materials needed by industry, commerce, educational institutions, and Government; provides advisory and research services to other Government agencies; and develops, produces, and distributes standard reference materials. The Institute consists of the Office of Standard Reference Materials and the following divisions:

Analytical Chemistry - Polymers - Metallurgy - Inorganic Materials - Reactor Radiation - Physical Chemistry.

THE INSTITUTE FOR APPLIED TECHNOLOGY provides technical services to promote the use of available technology and to facilitate technological innovation in industry and Government; cooperates with public and private organizations leading to the development of technological standards (including mandatory safety standards), codes and methods of test; and provides technical advice and services to Government agencies upon request. The Institute consists of a Center for Building Technology and the following divisions and offices:

Engineering and Product Standards - Weights and Measures - Invention and Innovation - Product Evaluation Technology - Electronic Technology - Technical Analysis

- Measurement Engineering - Structures, Materials, and Life Safety ${ }^{4}$ _ _Building Environment * Technical Evaluation and Application ${ }^{4}$ - Fire Technology.

THE INSTITUTE FOR COMPUTER SCIENCES AND TECHNOLOGY conducts research and provides technical services designed to aid Government agencies in improving cost effectiveness in the conduct of their programs through the selection, acquisition, and effective utilization of automatic data processing equipment; and serves as the principal focus within the executive branch for the development of Federal standards for automatic data processing equipment, techniques, and computer languages. The, Institute consists of the following dịvisions:

Computer Services - Systems and Software - Computer Systems Engineering - Information Technology.

THE OFFICE FOR INFORMATION PROGRAMS promotes optimum dissemination and accessibility of scientific information generated within NBS and other agencies of the Federal Government; promotes the development of the National Standard Reference Data System and a system of information analysis centers dealing with the broader aspects of the National Measurement System; provides appropriate services to ensure that the NBS staff has optimum accessibility to the scientific information of the world. The Office consists of the following organizational units:

Office of Standard Reference Data — Office of Information Activities - Office of Technical Publications - Library - Office of International Relations.

${ }^{1}$ Headquarters and Laboratories at Gaithersburg, Maryland, unless otherwise noted; mailing address Washington, D.C. 20234.

${ }^{2}$ Part of the Center for Radiation Research.

3 Located at Boulder, Colorado 80302.

- Part of the Center for Building Technology. 


\section{Cover Photo:}

Cynthia Main, Physical Science Aid, adjusting the wire in an

ultrasonic bonding machine in preparation for bonding.

Library of Congress Catalog Number: 73-600343

\section{National Bureau of Standards Special Publication 400-2}

Nat. Bur. Stand. (U.S.), Spec. Publ. 400-2, 109 pages (Jan. 1974)

CODEN: XNBSAV 
INTRODUCTION . . . . . . . . . . . . . . . . . . . . . 2

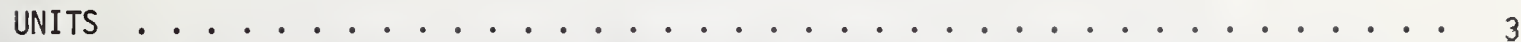

1.0 EQUIPMENT AND TECHNIQUES FOR MEASUREMENT AND CONTROL OF ULTRASONIC BONDING . . . . 4

1.1 Measurement Methods for UItrasonic Systems of Bonding Machines . . . . . . 4

1.1 .1 Modification and Use of a Capacitor Microphone . . . . . . . . . . 4

1.1.2 Magnetic Pickups for Tuning and Establishing Vibration Amplitude . . . 9

1.1.3 Laser Interferometer for Making Quantitative Measurements of Bonding Tool Vibration Amplitude . . . . . . . . . . . . . 11

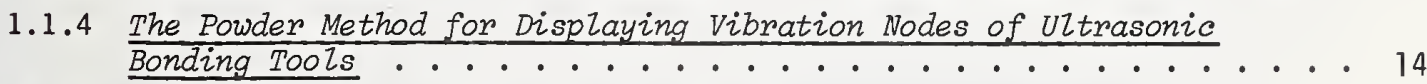

1.2 Techniques, Apparatus, and Test Methods for Assuring or Measuring

Bond Reproducibility .................. . . 16

1.2.1 An ULtrasonic Vibration Nondestructive Bond Screen . . . . . . . 16

1.2.2 An Automatic Recording Wire-Bond Hook-Pulling Apparatus . . . . . . 16

1.2.3 Hot Melt Glue Apparatus for Pulling a Single Wire-Bond . . . . . . . 18

1.2 .4 Wire-Quality Indentation Tester . . . . . . . . . . . . 19

1.2 .5 Bonding Force Measurement . . . . . . . . . . . . . . . . 19

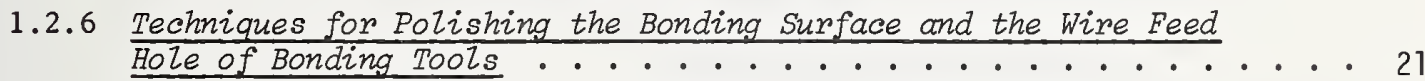

1.2.7 Sintering Temperature and Bondability of Aluminum Metallization . . . 22

2.0 THE ULTRASONIC BONDING MACHINE . . . . . . . . . . . . . . . . . 23

2.1 Setup and Checkout Procedures . . . . . . . . . . . . . . . 23

2.1 .1 Introduction . . . . . . . . . . . . . . . . . . 23

2.1.2 Initial Procedures . . . . . . . . . . . . . . . . . 23

2.1 .3 Daily Check out Procedure . . . . . . . . . . . . . . . . . 24

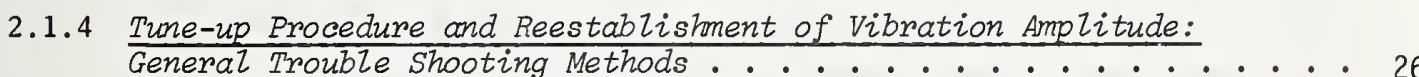

2.2 Some Mechanical Problems of Bonding Machines. . . . . . . . . . . . . . . 29

2.2.1 Low Frequency Motion and Bond Formation . . . . . . . . . . . 29

2.2.2 Motion Perpendicular to the Wire. . . . . . . . . . . . . 30

2.2.3 Motion Parallel to the Wire ................. 30

2.2.4 Self-Induced Motion in Bonding Machines ............ 33

2.2.5 Wire Clamp Problems ................... 36

2.3 Characterization of the Ultrasonic Systems Employed and Various

Problems Encountered in Their Use . . . . . . . . . . . . . 38

2.3.1 Temperature Characteristics of Transducers . . . . . . . . . . 38

2.3.2 Transducer Efficiency Variations Observed on Assembly Lines . . . . 38

2.3.3 Laboratory Setup for Measuring Transducer and Tool Vibration

Characteristics ..................... . 39

2.3.4 Bonding Tool Vibration Modes . . . . . . . . . . . . . 39

2.3.5 Effect of Loading on Tool Vibration Modes . . . . . . . . . . . 41 
2.3.6 Transducer-Mount Interaction Problems . . . . . . . . . . 4 41

2.3.7 Modification of the Tronsducer Q . . . . . . . . . . . 43

2.3.8 Resononce Dominated by a Critical Extension of the Tool . . . . . 45

2.3.9 Bonding Tool Vibration Amplitude Variations Resulting from

2.3.10 Effect of Power Supply Impedance on the $Q$ of the Ultrasonic System . . . . . . . . . . . . . . . . . 50

2.3.11 The Swept Frequency Mode of Power Supply Operation . . . . . . . 50

2.3.12 Studies of a Commercial Phase-Locked-Loop Ultrasonic Power Supply . . 55

3.0 IN-PROCESS BOND STUDIES, AND THE MECHANISM OF ULTRASONIC BONDING . . . . . . . 58

3.1 In-Process Bond-Quality Studies . . . . . . . . . . . . . 58

3.1 .1 Preliminary Studies . . . . . . . . . . . . . . 58

3.1 .2 Beat-Frequency Audio Monitoring . . . . . . . . . . . . . 59

3.1.3 Harmonic Content and Phased Burst Studies. . . . . . . . . . . . 61

3.1.4 Short Ultrasonic Bonding Tools . . . . . . . . . . . . . . 65

3.1 .5 Long ULtrasonic Bonding Tools . . . . . . . . . . . . . . 73

3.2 Studies of the Ultrasonic Bonding Mechanism . . . . . . . . . . . 73

3.2 .1 Bond Lift-Off; Laboratory Experiments . . . . . . . . . . . . 73

3.2 .2 Bond Lift-Off; Commercial Devices . . . . . . . . . . . . 76

3.2 .3 Ultrasonic Bonding Mechanism . . . . . . . . . . . . . 79

4.0 VARIOUS EFFECTS OF BOND GEOMETRY AND WIRE CHARACTERISTI $\mathrm{CS}$. . . . . . . . . . 80

4.1 Bond Failure Resulting from Device Power Cycling . . . . . . . . . . 80

4.1 .1 Theoretical Results . . . . . . . . . . . . . . 80

4.1 .2 Experimental Verification . . . . . . . . . . . . . 85

4.1.3 Conclusions: Loop Height Requirements . . . . . . . . . . . 85

4.2 The Effect of Loop Height on Bond Pull Strength . . . . . . . . . . . . . 86

4.3 A Bond Pull Strength Acceptance Criterion Based on Geometry . . . . . . . . 88

4.4 The Effect of Wire Elongation on Bond Pull Strength . . . . . . . . . . 90

4.5 Ultrasonic Bondability of Gold Wire . . . . . . . . . . . . . . 94

5.0 APPENDIX (Bonding Too 1 Vibration Modes). . . . . . . . . . . . . . 96

6.0 REFERENCES AND NBS PUBLICATIONS USED IN THIS REPORT . . . . . . . . . . . . . . 98

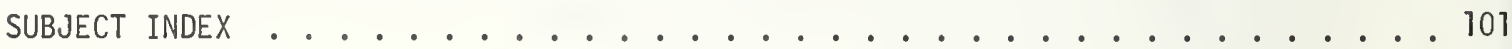




\section{PREFACE}

The wire bond evaluation work has been carried out under the NBS Electronic Technology Division's Joint Program on Methods of Measurement for Semiconductor Materials, Process Control, and Devices. The Program was undertaken in 1968 to focus NBS efforts to enhance the performance, interchangeability, and reliability of discrete semiconductor devices and integrated circuits through improvements in methods of measurement for use in specifying materials and devices and in control of device fabrication processes. These improvements are intended to lead to a set of measurement methods which have been carefully evaluated for technical adequacy, which are acceptable to both users and suppliers, which can provide a common basis for the purchase specifications of government agencies, and which will lead to greater economy in government procurement. In addition, such methods will provide a basis for controlled improvements in essential device characteristics, such as uniformity of response to radiation effects. 


\section{ACKNOWLEDGMENT}

The editor gratefully acknowledges the help of A. H. Sher, H. A. Schafft, H. K. Kessler, K. O. Leedy and J. C. French for their suggestions on the manuscript organization as well as their assistance in proof reading the final copy. I am especially grateful for the original editorial work of W. M. Bullis, editor of the Quarterly report series "Methods of Measurement for Semiconductor Materials, Process Control and Devices." The manuscript was expertly prepared by Miss B. S. Hope. 


\title{
MICROELECTRONIC ULTRASONIC BONDING
}

Selected Work From the NBS Microelectronic Wire Bonding Project

\author{
G. G. Harman, Editor
}

\section{ABSTRACT}

This report primarily comprises excerpts of the work done under the NBS ultrasonic wire bonding program that was previously published in 17 quarterly and other reports. The material is organized into subject groupings with the intention of presenting in convenient form sufficient information for making high quality ultrasonic wire bonds as well as imparting a basic understanding of the ultrasonic systems used. The work emphasizes problems and methods of solving them. To accomplish this, the required measurement equipment is first introduced. This is followed by procedures and techniques used in setting up a bonding machine, and then various machine- or operator-induced reliability problems are discussed. The characterization of the ultrasonic system and its problems are followed by in-process bonding studies and work on the ultrasonic bonding (welding) mechanism. The report concludes with a discussion of various effects of bond geometry and wire metallurgical characteristics. Where appropriate, the latest, most accurate value of a particular measurement has been substituted for an earlier reported one. Thus all of the included material is up to date.

Key Words: Bonding; degradation (wire bond); discrete devices; electrical interconnection; fabrication (wire bond); failure (wire bond); hybrid circuits; integrated circuits; microelectronics; reliability; semiconductor devices; testing (wire bond); ultrasonic bonding; wire bond. 


\section{INTRODUCTION}

This report describes work performed at NBS on ultrasonic wire bonding for use in microelectronic devices. The work has as its long-range objective to survey and evaluate methods for characterizing wire bond systems in semiconductor devices and, where necessary, to improve existing methods or develop new methods in order to detect more reliably those bonds which will eventually fail.

Most of the report has appeared in 17 quarterly and other reports which have been published as NBS Technical Notes. The number of the NBS Technical Note from which each portion of the report was extracted is given in brackets in the margin at the beginning of that portion. A list of these reports, including the page numbers of the wire bonding sections, is given with the references. Identification of those who performed the work is given at the end of each section, as has been the custom in the quarterly reports.

The material selected from the previously published Technical Notes was incorporated into this report with a minimum of rewriting. A new titling system was necessitated, new introductory and summary paragraphs were added along with conjunctive sentences and phrases. In general, the first reported development or use of a new technique was omitted if it was subsequently studied and reported in greater depth. However, references to the original work are included. When appropriate, the latest, most accurate value of a particular measurement has been substituted for an earlier reported one. Thus all of the included material may be considered up to date.

In order to keep this publication to a useful size, several wire bonding topics that appeared in the quarterly reports were omitted from this report. However, these are currently available or will soon be published as separate NBS Technical Notes. Prominent among these are the extensive work on ultrasonic ribbon wire bonding done by H. K. Kessler and A. H. Sher published as NBS Technical Note $767, *$ and the many aspects of the wire-bond pull-test by K. O. Leedy, C. A. Main and A. H. Sher. Omitted also were the complete mathematical solution of forces in the wire for the wire bond pull test, -as well as the various references to the bibliography and the comprehensive survey of the wire bonding literature by H. A. Schafft. These were recently published as NBS Technical Notes 593 and 726 respectively. ${ }^{+}$

* H. K. Kessler and A. H. Sher, Microelectronic Interconnection Bonding with Ribbon Wire. NBS Technical Note 767 (April 1973).

† H. A. Schafft, Wire-Bond Electrical Connections, Testing, Fabrication and Degradation - A Bibliography 1957-1971, NBS Technical Note 593 (Jan 1972). H. A. Schafft, Testing and Fabrication of Wire Bond Electrical Connections - A Comprehensive Survey, NBS Technical Note 726 (Sept 1972). 
The selected material is organized into four major subject groupings with the intention of presenting under one cover pertinent information developed at NBS on the process of ultrasonic bonding to make high-quality ultrasonic wire bonds. The required measurement equipment (magnetic pickups, bonding force measurement apparatus, etc.) is introduced first. This is followed by procedures used in setting up a bonding machine, and by a discussion of various machine- and operator-induced reliability problems. The characterization of the ultrasonic system of a bonding machine and its problems are followed by in-process bonding studies and work on the ultrasonic bonding (welding) mechanism. The report concludes with some new, previously unpublished, material on various effects of bond geometry and wire metallurgical characteristics.

\section{G. G. Harman, Editor}

October 1973

\section{UNITS}

During the four years of work covered in this report inevitably there have been editorial style and usage changes. This is especially evident with respect to units. Initially in the quarterly reports, the units commonly employed in the domestic American semiconductor industry were used. Later these common units (mixed English and metric) were followed in parentheses by the appropriate International System (S.I.) unit, and in some cases only International units were used. In this report the common unit will be given first, followed by the rounded value of the International unit in parentheses the first time that unit appears in each subsection. For any remaining unconverted length units it should be noted that $1 \mathrm{mil}=0.001 \mathrm{in} .=25.4 \mu \mathrm{m}$, and the common unit given in bond pull strength tests is grams force [1 $\mathrm{gf}=9.8$ millinewtons $(\mathrm{mN})]$. 

BONDING

1.1 Measurement Methods for UTtrasonic Systems of Bonding Machines

\subsubsection{Modification and Use of a Capacitor Microphone}

[573] Amplitude measurements of ultrasonic bonding tool motion by means of a capacitor microphone have been used to characterize the ultrasonic systems of bonding machines, diagnose problems, and measure the effects of. extraneous motion on the making of bonds.

A capacitor microphone, without any additions, modifications, or amplification of its output, can be used to tune ultrasonic wire (or flip chip) bonding machine transducers to their mechanical resonance. A 1/4-in. or 1/8-in. (6.4-or 3.2-mm) diameter microphone produces a substantial output voltage (approximately $0.1 \mathrm{~V}$ ) when brought close to the vibrating tool or transducer horn end. However, the movement of a person's hand or any other object within several inches of the microphone may disrupt the standing wave pattern and change the output amplitude. If such motion is avoided, and the background noise level is not high, the mechanical resonance frequency of the system may be obtained. However, it is generally necessary to obtain considerably more information than the mechanical resonance alone. In order to trouble-shoot and to reestablish vibration amplitude of ultrasonic wire bonding equipment it is essential to improve the spatial resolution of the microphone.

Capacitor microphones with 1/4-in. or 1/8-in. diameter diaphragms normally detect incident sound from relatively large angles. However, when measuring parameters of wire bonding systems it is desirable to measure the sound pressure emitted near the tip of a bonding tool, an area $\sim 1 / 50$ of the area of the microphone. In order to do this, the entrance to the microphone must be constricted by some means such as by a conical tip.

The design of a tapered conical tip for focusing sound into the microphone is subject to many practical constraints and uncertainties. Generally, the volume of the cone should be kept as small as practical in order to minimize standing waves. Ideally, the cone is constructed of thin-walled steel. However, almost any continuously tapered material may be used. For example, it is completely adequate to construct a cone that has the necessary physical dimensions required for making the measurement and then insert appropriate damping material to eliminate standing

Such units are available from B and K Instrument Company, Cleveland, Ohio. Other capacitor microphones may serve the purpose just as well but were not available during this work. 
waves. A simple and satisfactory cone may be constructed by wrapping several layers of aluminum foil around a tapered mandrel, such as the end of a sharpened pencil. Even the plastic cone from the end of a tube of silicon rubber can be used.

In order to constrict the opening as well as to protect the small end of the steel cone from damage caused by physical contact with hard objects, such as tungsten carbide bonding tools, the cone is usually coated with a silicon rubber material (B in Fig. 1-1). A threaded metal sleeve that screws onto the microphone is bonded on the large end of the cone. The cone is screwed onto the microphone and is now ready to be used for simple measurements, such as peak tuning the mechanical resonance of a bonding tool.

If reproducible amplitude measurements are required, a small length, $[\sim 0.05$ in. $(1.3 \mathrm{~mm})]$, of a hypodermic needle, typically in the size range of \#20 to 30 is inserted and bonded into a needle hole in the silicon rubber (in A and B of Fig. 1-1). For maximum signal strength and reproducibility this needle must lie straight along the axis of the cone.

The total extension of the cone tip in front of the microphone is about $1 / 2$ in. to $3 / 4$ in. for a $1 / 4$ in. diameter microphone and about $3 / 8$ in. for $1 / 8$ in. diameter microphone. These dimensions are not critical. The acoustical pickup area is defined by the hole in the hollow needle, (A in Fig. 1-1) which is typically chosen to be from 0.004 to 0.15 in. (0.1 to $0.38 \mathrm{~mm}$ ) in diameter.

Microphone tapered tips made in the manner described above provide essentially complete rejection of all ultrasonic signals not entering through the entrance hole when this hole is positioned a few mils or less from the source. Extraneous ultrasonic sound waves are prevented from reaching the microphone diaphragm primarily by reflection from the outer surface of the cone. Audible sound, particularly if it contains low frequency components, may be transmitted through the cone walls. A simple, highpass R-C filter (Fig. 1-2) can be placed at the output of the microphone and preamplifier to eliminate such background interference.

For studying the vibration along the entire length of a bonding tool (Section 2.3.4) an amplitude reproducibility of about \pm 5 percent is essential to obtain meaningful results. This is achieved in the following manner. Both the source transducer and the microphone are held in rigid mounts, and the microphone is moved with a micrometer stage. In a given vertical position, the steel tip of the microphone is advanced until it just touches the bottom of the vibrating tool as shown in Fig. 1-3. The instant of contact is clearly evident because "hash" appears on the oscilloscope display of the microphone output. Then the tip is carefully retracted until the "hash" just disappears and a clear sine wave is seen. A reproducible signal (50 to $100 \mathrm{mV}$ ) is obtained with this technique. Figure 1-4 shows a typical microphone holder and how it is mounted on a bonding machine.

(G. G. Harman) 


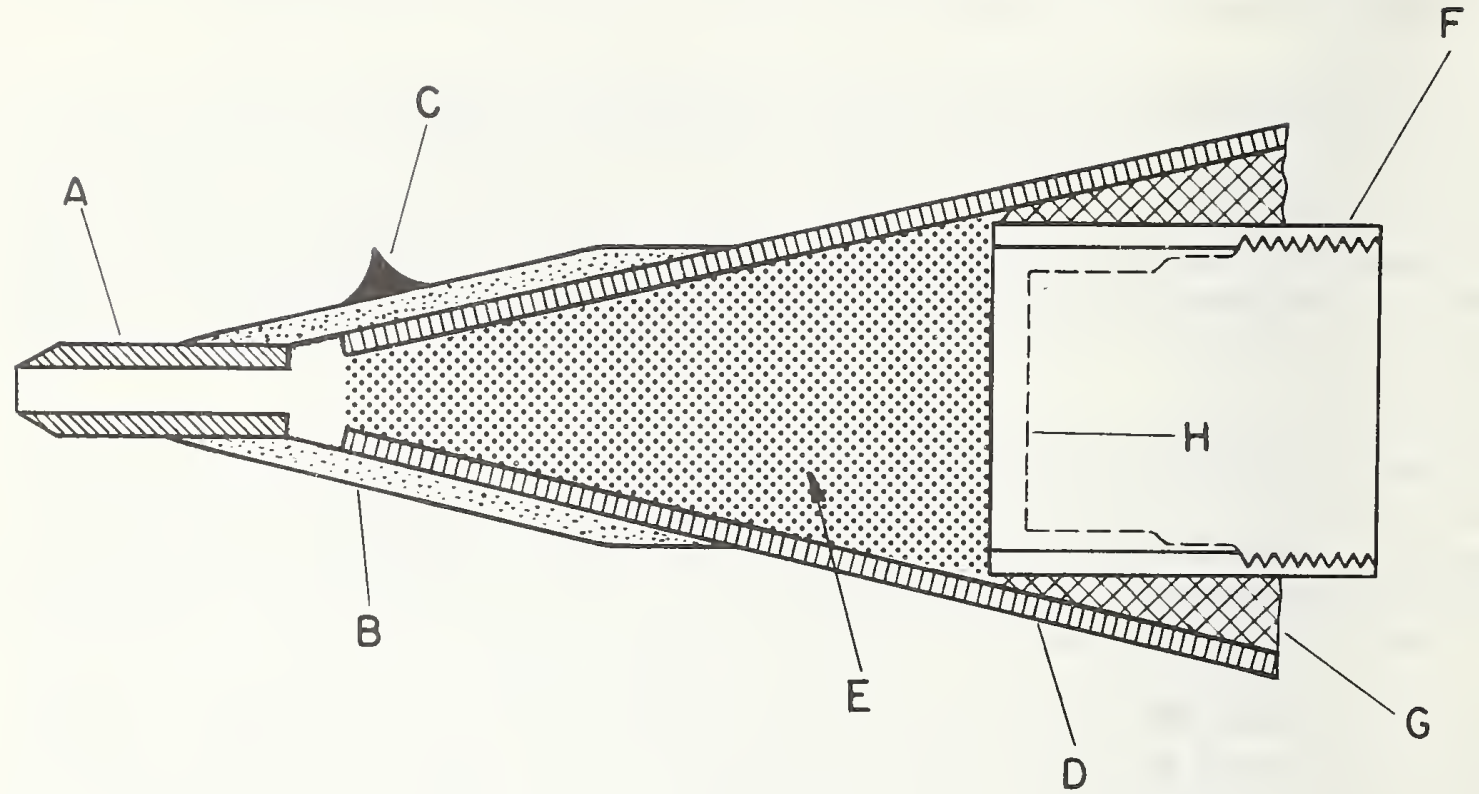

Fig. 1-1. Cross-sectional view of a high-resolution extension tip for the capacitor microphone.

A: Section of hypodermic needle. This can be made from size \#20 to 30 but most units were made from \#27 or 29 needles.

B: Self-leveling silicone rubber coating

C: Red or black silicone rubber dot for reproducible orientation of A with respect to the bonding tool

D: Steel or aluminum cone

$E$ : Cotton or open-pore plastic foam damping material with low reflection of the ultrasonic signal

F: Stainless steel threaded sleeve (typically made from a microphone protective grid)

G: Epoxy or silicone rubber bond between the sleeve, $F$, and the aluminum cone, D. (This bond must be tight or stray signals can enter the microphone from the rear.)

$\mathrm{H}$ : Dashed lines indicate position of microphone 


\section{MICROPHONE \& FET PREAMP

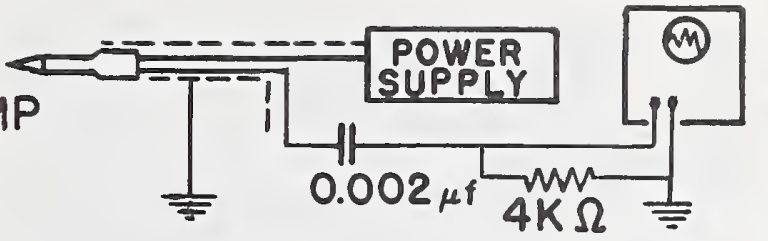 \\ OSCILLOSCOPE DISPLAY}

Fig. 1-2.

A simple high pass filter, consisting of a $0.002 \mu \mathrm{F}$ capacitor and a $4 \mathrm{k} \Omega$ resistor, can be inserted between the microphone preamplifier and the oscilloscope to eliminate audible sound.

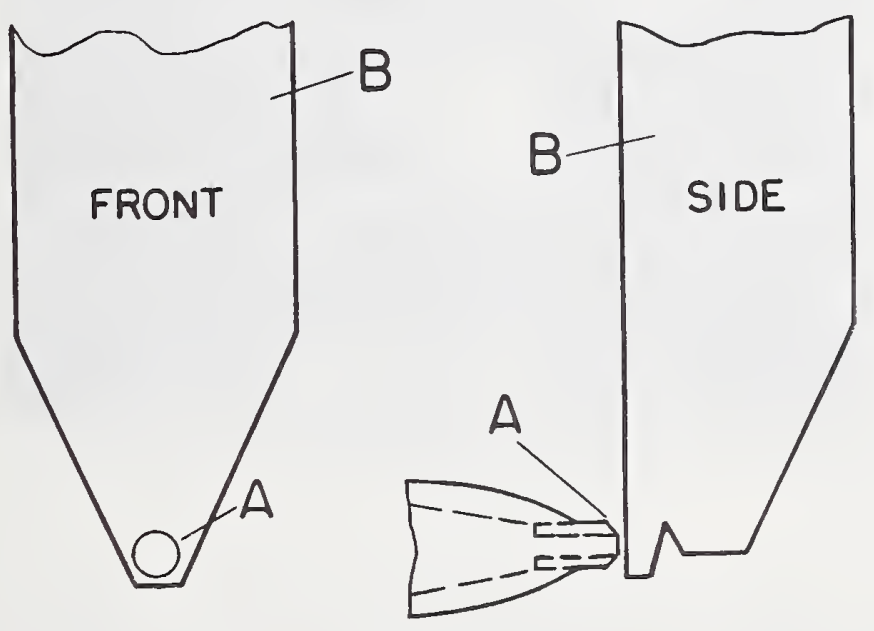

Fig. 1-3. Positioning of the microphone tip on the bonding tool. (A) is the hollow steel needle. (B) is the bottom section of an ultrasonic bonding tool. The solid circle (front view) is the typical position for measuring vibration amplitude. 
(A)

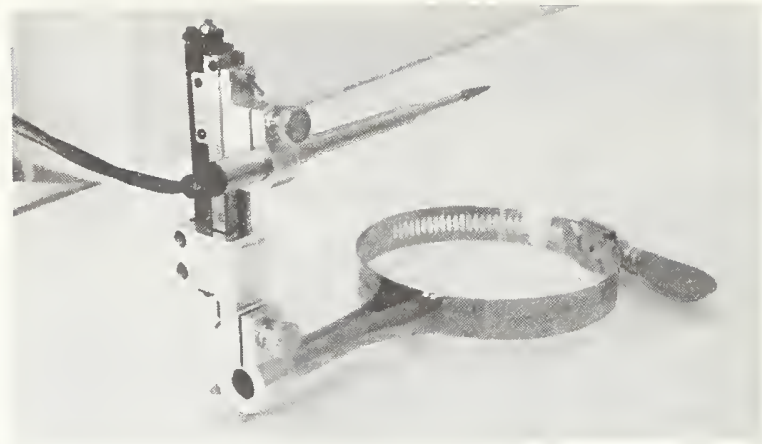

(B)

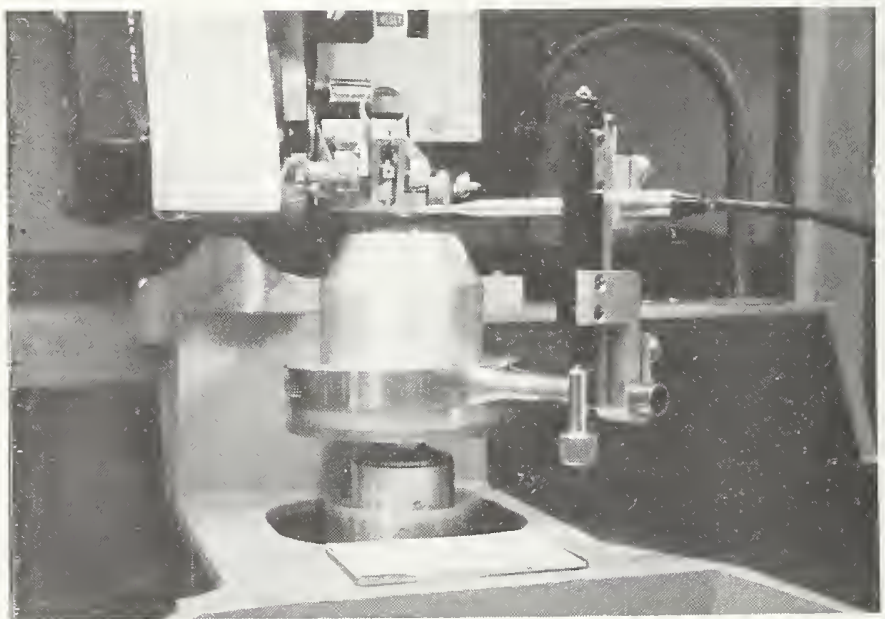

Fig. 1-4. (A) A simple fixture that is made to clamp directly on the bonding machine workstage. The $\mathrm{x}$ and $\mathrm{y}$ movements are obtained by using the bonding machine chessman positioner. The $z$ movement is made from an inexpensive microscopegraduated mechanical stage and is adjusted with the knob at the bottom of the positioner [more clearly shown in (B)]. This picture displays a $1 / 8$ in. diameter microphone with a tapered steel tip.

(B) The same fixture and microphone as in (A) mounted on a bonding machine workstage. (Note: for purposes of clarity the microphone is not shown directly in line with the transducer horn as it would normally be in use.) 


\subsubsection{Magnetic Pickups for Tuning and Establishing Vibration Amplitude}

microphone system described in Section 1.1.1 has been used successly for the laboratory study of the ultrasonic systems used in wire ding. It has also been used on semiconductor device assembly lines to ie and trouble-shoot ultrasonic wire bonding machines. However, it has reral disadvantages. One important drawback is that the precision er tips necessary for reproducible measurements are difficult to make are not available commercially at present. Secondly, microphones are atively expensive, delicate instruments that must be handled with care qualified technical personnel in order to prevent damage. Ideally, a suring device for assembly line use should be cheap and rugged.

sideration of these necessary characteristics led to the reinvestigan of magnetic pickups. It was previously noted in this laboratory is Tech. Note 495, p. 25) that magnetic pickups were sensitive to the ration of bonding tools. (Davis [1] had independently reported a ilar observation.) Compared with the microphone, the signal from the metic detector is relatively insensitive to its distance from the rating tool. This feature makes it easier to use in reestablishing tool tip vibration amplitude after changing tools or in retuning. ever, for the same reason, it is less useful than a microphone for intigating the mechanical stability and trouble shooting the bearings of bonding machine.

electrical output of these pickups is in the range of 10 to $20 \mathrm{mV}$ n measuring typical tool vibration amplitudes used in bonding 0.001 in (um) aluminum wire.* This represents tool motion in the vicinity of to $80 \mu \mathrm{in}$. ( 1 to $2 \mu \mathrm{m}$ ) peak-to-peak displacement. The spatial resoion, at the bonding tool, of the presently available magnetic pickups about 0.05 in. $(1.25 \mathrm{~mm})$, which is adequate for tuning purposes. A ical device has concentric pole pieces as shown in Fig. 1-5A. The ts are approximately $1 / 2$ in. (12.5 mm) long and their outside surface threaded with a 10-32 NF thread as shown in Fig. $1-5 \mathrm{~B}^{\dagger}$ for magnetic pickups, one unmounted, and the other mounted on the end of aluminum rod. This rod can be substituted for the microphone in the cture shown in Fig. 1-4. They may be mounted on a bonding machine with same fixtures used for holding microphones (Fig. 1-4) as shown in 5. 1-5C. Other smaller, transistor-sized fixtures are possible. The netic pickups are sensitive to stray magnetic fields. Thus, if a

\section{See footnote page 11}

Such units are available from Electro Corp., Sarasota, Florida. Other magnetic units may serve the purpose just as well but were not available during this work. 
(A)

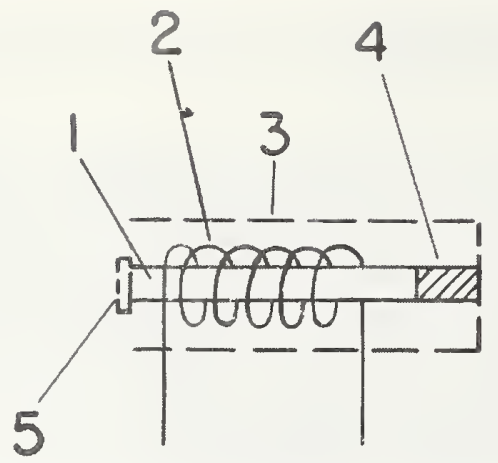

(B)

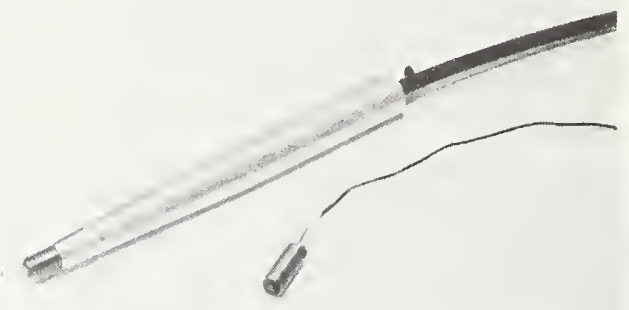

(C)

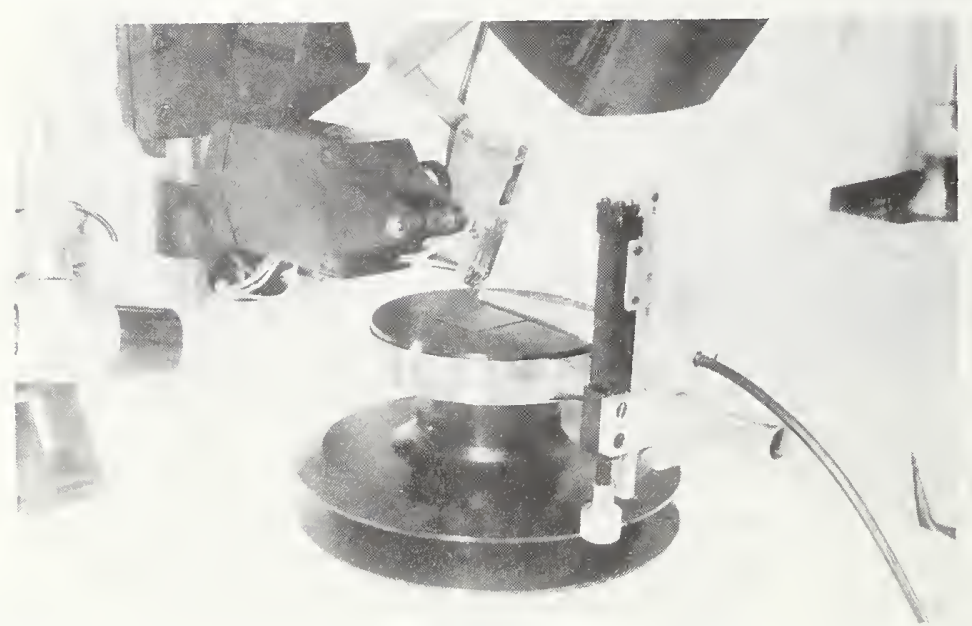

Fig. 1-5. (A) Construction of a typical magnetic pickup. (1) magneticmaterial core, (2) induction coil, (3) magnetic-material case, (4) permanent magnet, (5) thin (0.001 in.) mylar tape film to prevent the vibrating bonding tool from damaging the pickup core. (When applying the film, care must be taken to insure that no air bubbles exist at the tape-pickup interface.) (B) Photographs of magnetic pickups used for measurements on ultrasonic bonding machines. The small unit is the detector alone, the larger unit shows the detector mounted in a rod that fits in various microphone mounts shown earlier in Fig. 1-4. (C) A magnetic pickup mounted on a bonding machine ready for measuring the bonding tool vibration amplitude. 
magnetic chuck is used on the work stage, its field should be shunted. The center pole piece of the pickup should be cleaned occasionallv to remove attached magnetic or other particles.

The technique for tuning and reestablishing the vibration amplitude of the bonding tool is to bring the magnetic pickup close to the tool and about 0.1 in. $(2.5 \mathrm{~mm})$ above its tip and then adjust the pickup vertically downward until the signal amplitude is maximum. This amplitude represents the averaged motion of the lowest vibration loop of the tool. One should be sure that a higher vibration loop is not measured, (see Appendix for the vibration pattern of tools).

Some magnetic pickups have an asymmetrical amplitude response in the horizontal direction. This should be investigated when the pickup is first placed in service. A mark centered at the maximum response position will allow quick, reproducible, horizontal positioning of the pickup with respect to the tool. After use, the sensitivity of a magnetic pickup may decrease suddenly by 30\%, presumably due to dropping or other damage to the internal magnet. Therefore, it is desirable to keep a second calibrated pickup available to confirm the cause of any sudden output decrease.

The above procedure should provide a reproducible amplitude within $15 \%$ when normal tungsten carbide tools from the same manufacturer are compared.*

(H. K. Kessler)

\subsubsection{Laser Interferometer for Making Quantitative Measurements of Bonding Tool Vibration Amplitude}

A laser interferometer system was constructed to make quantitative measurements of the vibration amplitude of the ultrasonic bonding tools. The principal of the interferometer has been described by Clunie and Rock [2]; specific details were obtained from Martin [3]. The system uses a heliumneon milliwatt laser in which energy is emitted from both ends (approximately $90 \%$ from the front and $10 \%$ from the rear). Part of the light from the front is reflected back from the bonding tool, reenters the laser tube, modulating the inverted electron population, causing a significant

* Recently (1973) one bonding tool manufacturer has used a different tungsten carbide stock that presumably has more cobalt binder. The output from a magnetic detector may be five times higher from these tools than from normal ones vibrating with the same amplitude (as determined by a laser interferometer, Section 1.1.3). 
change in the light emitted from the rear of the laser tube. This rear beam is detected by a high-frequency-response silicon diode. Its output is displayed on an oscilloscope. The system has

produced signals from a vibrating bonding tool which were similar to those shown previously from a conventional laser interferometer (NBS Tech. Note 520, p. 42).

[571] The helium-neon laser operates at a wavelength of $0.6328 \mu \mathrm{m}$; thus each complete interference fringe is equivalent to a bonding tool displacement of $12.5 \mu i n$. $(0.316 \mu \mathrm{m})$. A typical interferometer pattern obtained by reflecting the laser beam off a vibrating bonding tool is shown in Fig. 1-6. The picture was made by photographing a single sweep of the oscilloscope beam. Approximately 3.2 fringes appear between the turn-around or end points of the motion. This represents a total displacement of 40 uin. $(1 \mu \mathrm{m})$.

The interferometer system has been used to calibrate ultrasonic power supply output control dial settings as a function of $60 \mathrm{kHz}$ bonding tool motion. A typical example of such a calibration is shown in Fig. 1-7. Amplitudes between 30 and $60 \mu \mathrm{in}$. (0.75 and $1.5 \mu \mathrm{m})$ peak-to-peak are typically used in making $1 \mathrm{mil}(25 \mu \mathrm{m})$ diameter aluminum wire bonds to aluminum bonding pads.

[592] In use the laser is mounted on a stabilized, portable tripod platform having $x-, y-$, and $z$-axis micrometer positioners as shown in Fig. 1-8. The interferometer output is displayed on an oscilloscope (not shown in the figure). The system can be set up and optically aligned on any bonding machine or experimental setup in approximately 5 minutes. A complete calibration of bonding-tool-tip vibration-amplitude as a function of power supply control setting takes only 5 to 10 minutes of additional time.

When using the above system the laser is not physically attached to the bonding machine. Therefore, small, low-frequency room vibrations, in the order of several microinches, will change the output waveshape at the end, or turn-around points of the tool motion. This makes it impossible to display the laser output as a repetitive oscilloscope pattern and requires that data be taken from a single sweep of the oscilloscope beam. This can be done either with a memory oscilloscope that has a sufficientl? fast writing rate or by photographing the interferometer pattern from a standard oscilloscope trace. Photographs have been used in the present study. No room motion is evident during the short (approximately $20 \mu s$ ) sweep period, and the photograph serves as a record that may be preserved for future reference.

In addition to measuring the absolute displacement of bonding tools, the laser has been compared with the capacitor microphone for trouble shooting bonding machines. Since it is not practical to design many different mounting systems to attach the laser rigidly to the variety of bonding machines available, measurements are restricted to a single oscilloscope sweep and thus are not suitable for trouble-shooting and diagnostic work. 


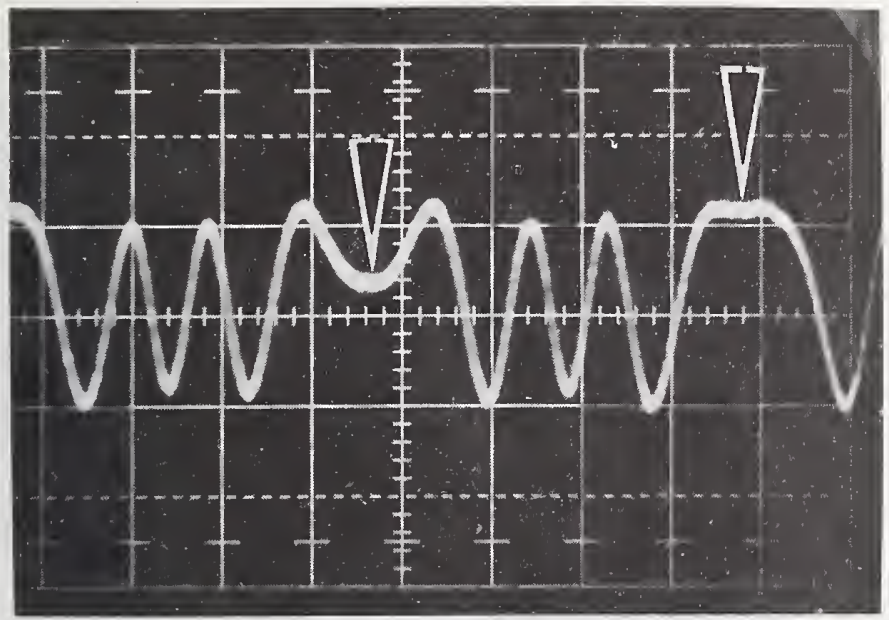

Fig. 1-6. Oscilloscope trace of a typical laser interferometer pattern obtained by reflecting a $0.6328-\mu \mathrm{m}$ laser beam off the tip of a bonding tool. The 3.2 fringes between the bonding tool "turn around" or end points (shown by the arrows) represent a vibration amplitude of 1.0 $\mu \mathrm{m}$ (40 $\mu$ in) .

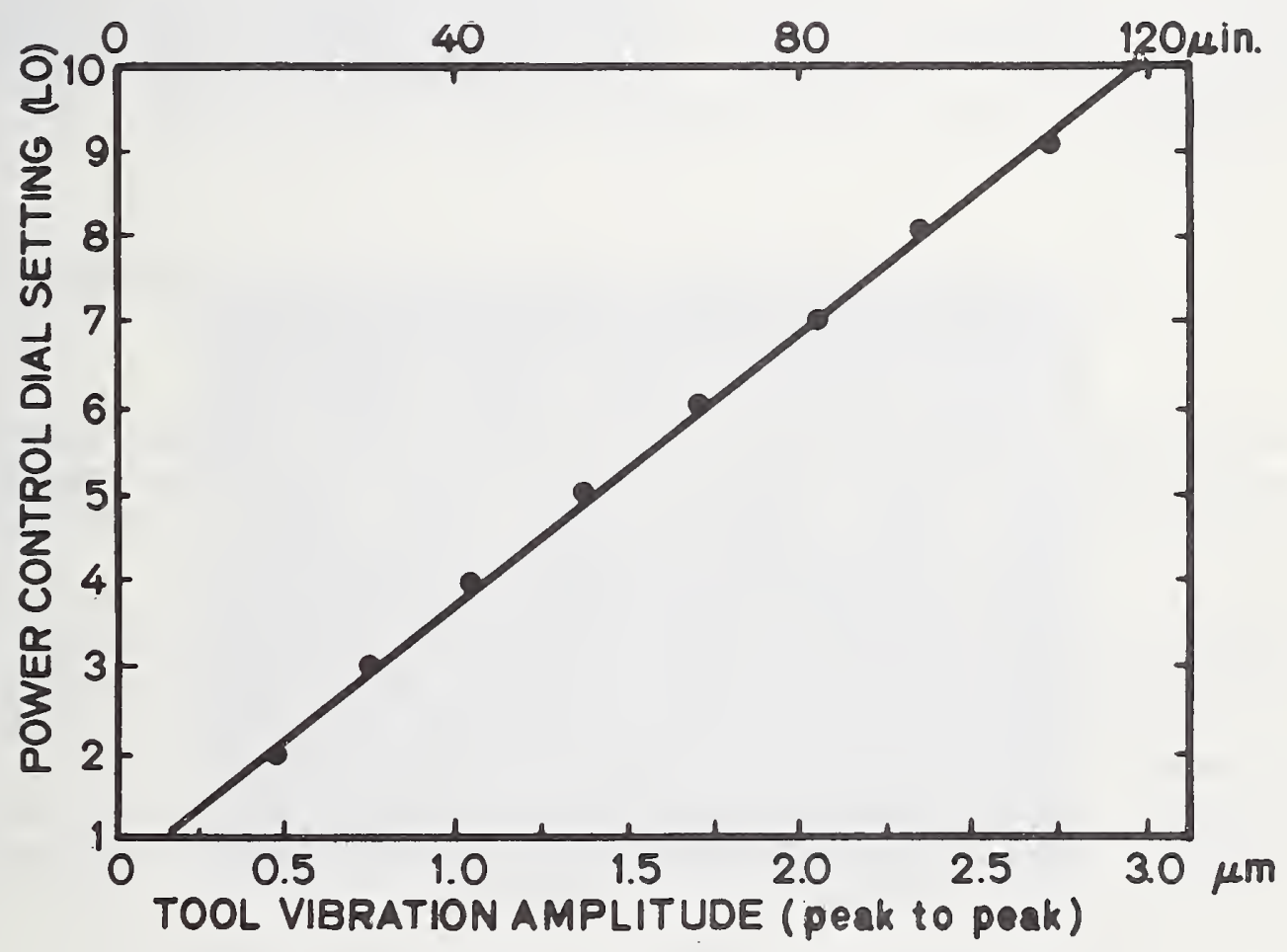

Fig. 1-7. U1trasonic power supply output control dial setting as a function of the vibration amplitude of the bonding tool tip. (The driving frequency is $60 \mathrm{kHz}$; the control is set to the LO position.) Different power supply-transducer combinations may produce significantly different amplitudes for the same dial settings and bonding tool extension. One combination produced $200 \mu \mathrm{in}$. $(5 \mu \mathrm{m})$ apparently under the same conditions. 
An example is shown in Fig. 1-9, a single exposure photograph from a double beam oscilloscope. The upper pattern is a repetitive trace from a capacitor microphone, the lower is a single trace from the laser interferometer. Both instruments were directed toward the same bonding tool on which the set screw had been loosened. The microphone pattern clearly indicates that a problem exists, but the single interferometer trace below does not encompass enough bonding tool vibration cycles to detect the problem. Thus at present the laser is used exclusively for absolute calibration of bonding tool motion. This calibration can then be transferred to more portable measuring devices, such as magnetic pickups and capacitor microphones.

(G. G. Harman)

One problem with the small commercial laser employed in the present interferometer is that it is primarily a multimode device. For interferometry, the unit must lase in a single mode. Previously the laser had been tuned to maximum output and then detuned to the TEM $\mathrm{OO}_{\text {mode. This is }}$ a critical adjustment subject to both amplitude and mode drifting. Even a few percent of a higher mode creates an unacceptable noise level in the detector output. At the suggestion of the manufacturer, special end plates were made to increase the laser cavity length by $1 \mathrm{~cm}$. The mirrors were reinstalled and the laser retuned. This resulted in a stable laser that operates on $1 \mathrm{y}$ in the $\mathrm{TEM}_{\mathrm{OO}}$ mode and therefore makes a better interferometer.

(G. G. Harman and A. W. Stallings)

\subsubsection{The Powder Method for Displaying Vibration Nodes of Ultrasonic Bonding Tools}

[495] It has been reported that the resonant nodes of vibrating bonding tools can be delineated with lycopodium powder [4]. Other powders, that may be more readily available, have been found to perform the same function [5].

The technique for using either of these powders is to dip an artist's brush into the powder and lightly dust it on the bonding tool. Then a very low amount of ultrasonic power is briefly applied, causing the powdes to drop off everywhere except on the nodes. The application of too much ultrasonic power or for too long a time may cause all of the powder to drop off. The powder method can only be used to measure the unloaded vibration nodes, since the nodes shift upward during bonding and all of the powder will drop off (see 5.0 Appendix).

(H. K. Kessler) 


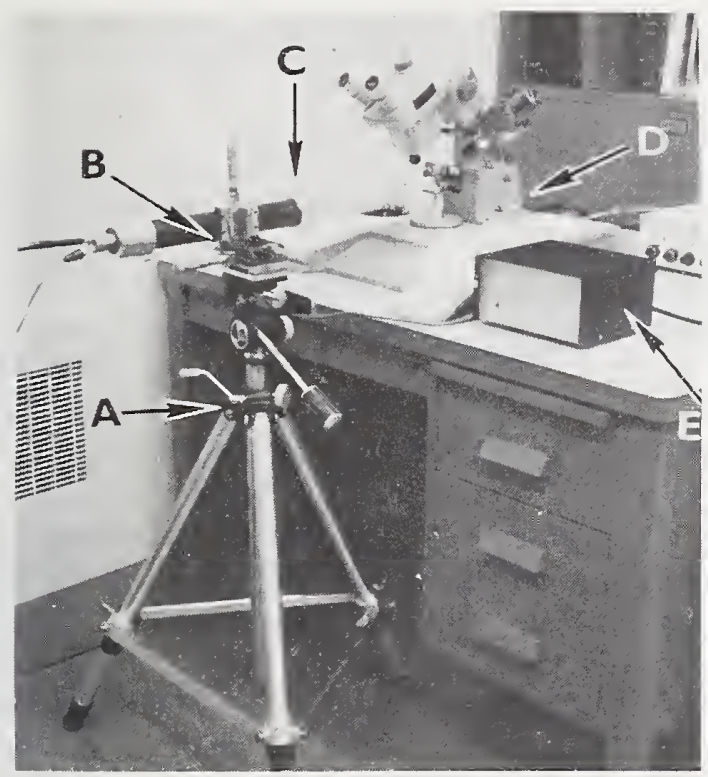

A - HEAVY DUTY TRIPOD WITH STABILIZED BASE

$B-X_{-}, Y_{-}$, AND Z-AXIS MICROMETER

ADJUSTMENTS FOR AIMING THE LASER

C - LASER SYSTEM WITH FOCUSING OPTICS

NEAREST THE BONDING MACHINE AND

DETECTOR AT THE REAR

D - BONDING MACHINE

E - LASER POWER SUPPLY

Fig. 1-8. Laser system set-up for calibrating ultrasonic bonding tool vibration amplitude.

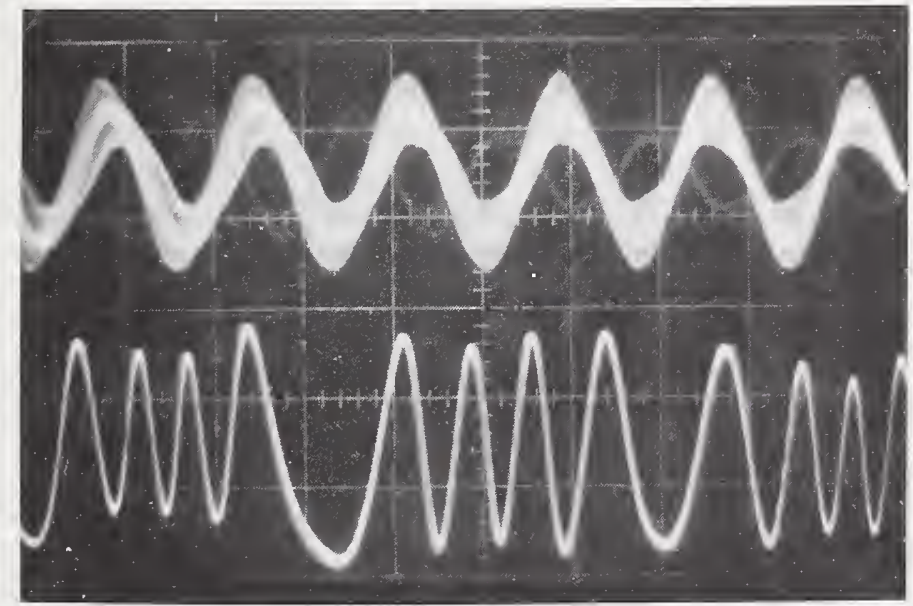

Fig. 1-9. A comparison between the sutput of a microphone and a laser interferometer simultaneously monitoring the same loose bonding tool. The upper curve is the output of the capacitor microphone and encompasses about five repetitive traces with approximately 5.5 tool motion cycles in each trace. Distortion of the wave shape is obvious. The horizontal scale is approximately $10 \mu \mathrm{s} / \mathrm{div}$. The lower curve is the laser interferometer output resulting from a single oscilloscope trace covering approximately 1.5 tool motion cycles. The horizontal scale is approximately 2 us/div. 


\subsubsection{An Uttrasonic Vibration Nondestructive Bond Screen}

[555] The use of ultrasonic excitation on complete transistors and integrated circuits in a liquid bath as a failure screen has been reported [6]. A novel method for testing individual bond-pair quality which is based on a different form of ultrasonic excitation was devised. This test is performed on an ultrasonic bonding machine by touching the bond loop with the front tip of a bonding tool, as shown in Fig. 1-10, and then applying ultrasonic power to the tool with the power supply control set near its maximum value. The vibration which is induced in the loop causes many bonds to lift off or break at the heel. Presumably, these bonds are weak ones which would eventually fail. In contrast to these, ultrasonic bonds made with 0.001 in. $(25 \mu \mathrm{m})$ diameter aluminum wire having power, time, and pressure settings adjusted to achieve maximum bond-pull strength, yield bonds with pull strengths in excess of 6 grams force even after exposure to such ultrasonic excitation for more than $10 \mathrm{~s}$. In addition to bond quality screening, the method appears to offer a means of assessing damage inflicted on a bond pair by prestressing or other physical means of evaluating bond quality. Although there is qualitative agreement with bond pull data on similar bonds, the method has not been completely evaluated. Optimum values have not yet been established for frequency, ultrasonic power, time of exposure to the ultrasonic vibration, and probe design. As yet there has been no statistical correlation between failure observed by this test and that observed by other tests. (G. G. Harman)

\subsubsection{An Automatic Recording Wire-Bond Hook-Pulling Apparatus}

[560] A new bond puller, pictured with its associated electronic equipment in Fig. 1-11, was completed. The puller was designed so that the bond pull rate could be varied by a factor of more than 10 . The pull strength in grams force, is read out with a specially constructed open core transformer-magnetic pickup system. Data are plotted on an $x-y$ recorder as straight lines with length in the $y$-direction proportional to the output signal. After each bond is pulled, the recorder pen is stepped along the $x$-axis. The new wire pulling system has increased both the speed and accuracy of wire bond pulling and data recording.

(H. K. Kessler and A, W. Stallings) 


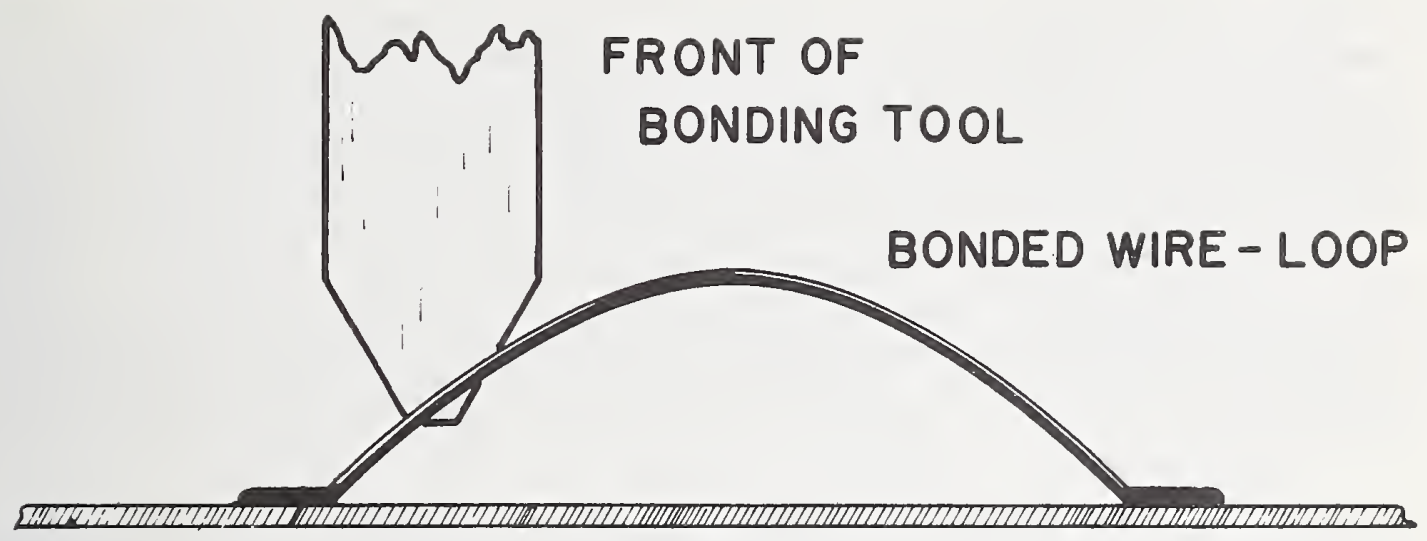

Fig. 1-10. Placement of bonding tool on bond loop for ultrasonic vibration screening test

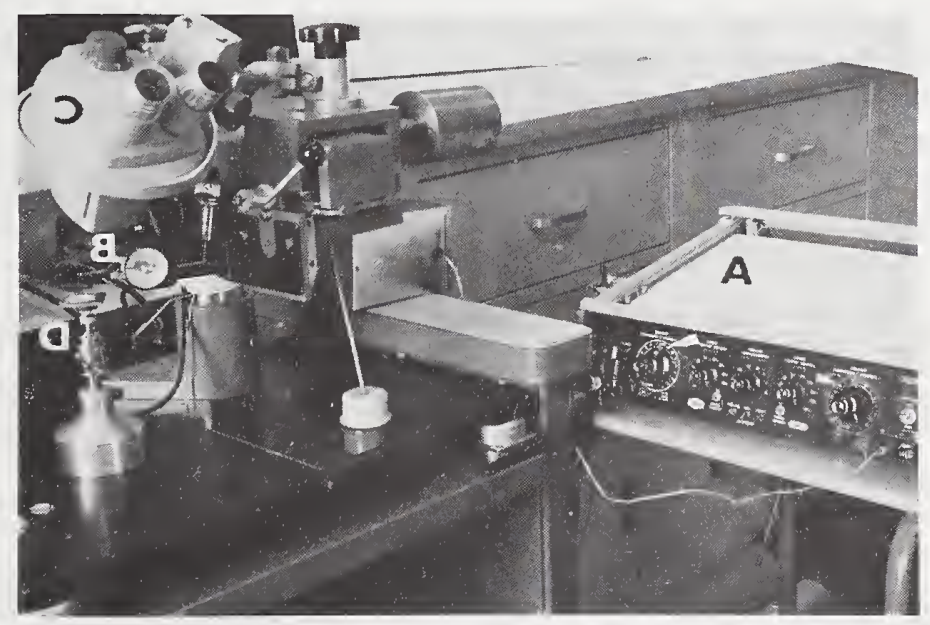

Fig. 1-11. Automatic wire bond pulling equipment.

$A-x-y$ recorder for automatic data recording.

B - Gram gage with magnetic sensing device.

C - Optical microscope.

D - Pedestal for mounting bonds to be pulled. 
[488] In order to determine the strength of a single bond, it has long been assumed that the wire must be pulled at the angle the bond was made in order to minimize perturbing effects such as peeling of the bond which is pullangle dependent. For a thermal compression bond this direction is perpendicular to the surface, and for an ultrasonic bond it is typically $30^{\circ}$ from the surface. As generally practiced, bond pulling is performed with a hook placed somewhere near the middle of the loop. However, in devices the die and post are frequently at different levels and the exact loop height and bond length are seldom known. Thus it is almost impossible to determine the actual direction and magnitude of the force applied to the bond.

A very simple method has been devised to grip the wire without deforming it so that it can then be pulled in any desired direction. In order to do this, a hairpin-shaped loop of 5-mil (125- $\mu \mathrm{m})$ diameter nichrome wire was electrically insulated and attached to the arm of a gram gage. The tip of the loop was electrolytically etched down to a $2-\mathrm{mil}(50-\mu \mathrm{m})$ diameter. This tip was then bent in the shape of a foot. A heating power of about $0.3 \mathrm{~W}$ was dissipated in the wire and the tip was wetted with a small dab of a special hot-melt glue. In operation the heated tip of this hot-melt puller is lowered over the free end of a wire that has its other end bonded to a substrate. The power is turned off, the glue cools and the bond is pulled. The thermal time constant of the system is a fraction of a second, therefore, little time is lost during heating or cooling. The choice of glue is very important. It is desirable to use one that has a discrete melting point (preferably below $200^{\circ} \mathrm{C}$ so that it does not anneal the wire) and that is quite hard when cold. Most such glues will bond well to a 5 mil length of 1 mil diameter aluminum wire. However, gold wire is more difficult for glues to grip, and larger bonding areas have been required to obtain sufficient strength for pulling the bond.

The hot-melt puller can also be used to test metallization adherance to a substrate. Considering an appropriate glue as having several thousand pounds per square inch tensile strength, the force applied to an aluminum metallization will be higher than is applied by the usual adhesive tape tests and can be used to test a much smaller, controlled area.

The hot-me1t puller has been used to measure the tensile strength of 1 mil aluminum bonding wire. In initial tests with wire from a single spool, repeated pulls to rupture on the same (decreasing) lengths of wire gave approximately the same tensile strength for each successive pull. Further work would be useful to substantiate this result. (G. G. Harman) 
A wire indentation tester was constructed. The system is designed to simulate the bonding operation insofar as the wire characteristics are concerned. The wire is indented at regular intervals by a wedge with dimensions similar to those of a bonding tool. A fixed force is applied to the wire each time and the depth of impression is measured by a displacement sensor, and recorded. Any irregularities can be readily observed and hence the pressure deformation qualities of the wire assessed.

A series of impressions made on three different grades of 1 mil (25 $\mu \mathrm{m})$ diameter aluminum ( $1 \%$ silicon) wire is shown in Fig. 1-12. Each line represents one impression. Impressions were made each $1 / 4$ in. (6.3 mm) for a distance of about $12 \mathrm{in.}(0.3 \mathrm{~m})$. The differences in uniformity can be clearly seen by the variations at the top of the repeated traces. The higher the trace, the smaller the impression and the harder the wire. Further experiments must be conducted to establish the optimum depth of impression to produce the maximum resolution. No correlation was found between variability of the wire impressions and the average tensile strength of the wire as measured with the hot-melt-glue puller (see Section 1.2.3). The wire specified as high-quality for ultrasonic bonding exhibited considerably less variability in breaking strength measurements than the other two samples which showed about the same variability when measured with the puller.

Several problems have been observed that are associated with the high sensitivity of the displacement sensor required in this application. These include slow drift-of the tester assembly due to temperature changes, drift of the recorder and other instruments, and insufficient dust shielding. Thus far no method has been devised for calibrating the depth of impression on an absolute basis to within the desired $5 \mu$ in $(0.13 \mu \mathrm{m})$. Therefore, the data must be compared on a relative basis. Additional development is necessary before the wire tester can be used to evaluate bonding wire quantitatively.

(H. K. Kessler)

\subsubsection{Bonding Force Measurement}

The bonding force is the most critical parameter in the ultrasonic bonding schedule. Small changes in the value of the bonding force can markedly affect the nature of the bond. Yet the bonding force is measured on most bonding machines by a hand-held gage, and the value of force obtained depends greatly on the skill of the operator. Therefore, the force gage mounting fixture shown in Fig. 1-13a was developed. To calibrate and adjust the force, the force gage assembly is clamped onto the work stage, and the power to the bonder is switched off. The bonder cam assembly is turned by hand through the first search position until the bonding tool touches the substrate, then the force gage arm is positioned horizontally in front of the transducer horn close to but not touching the bonding tool (see Fig. 1-13b). The transducer is then lifted approximately 10 to 


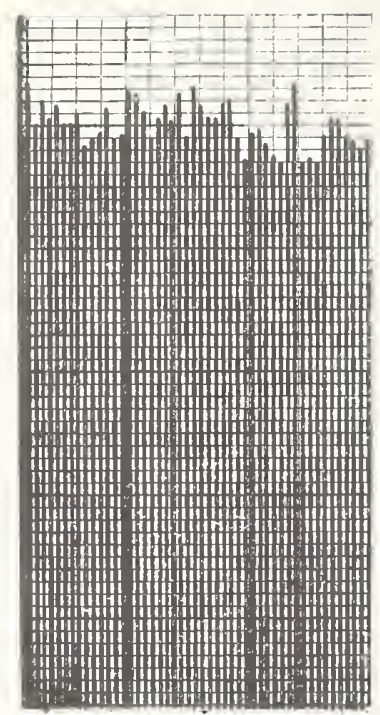

a. Wire rejected by a device manufacturer.

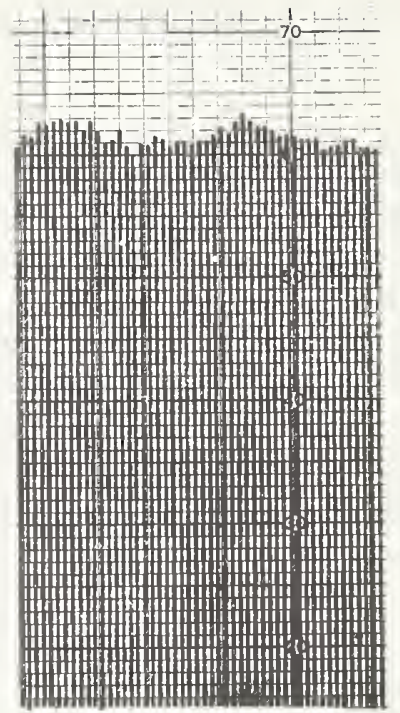

D. Wire not specified as ultrasonic bonding quality.

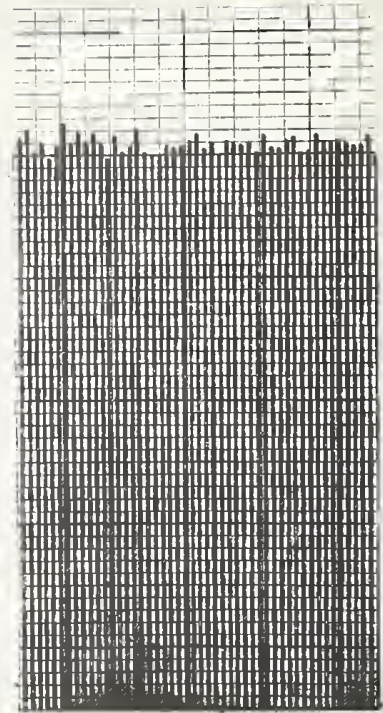

c. Wire specified as high-quality for ultrasonic bonding.

Fig. 1-12. Recorder traces of impressions made in three different specimens of 1-mil $(25 \mu \mathrm{m})$ diameter aluninum ( $1 \%$ silicon) bonding wire.
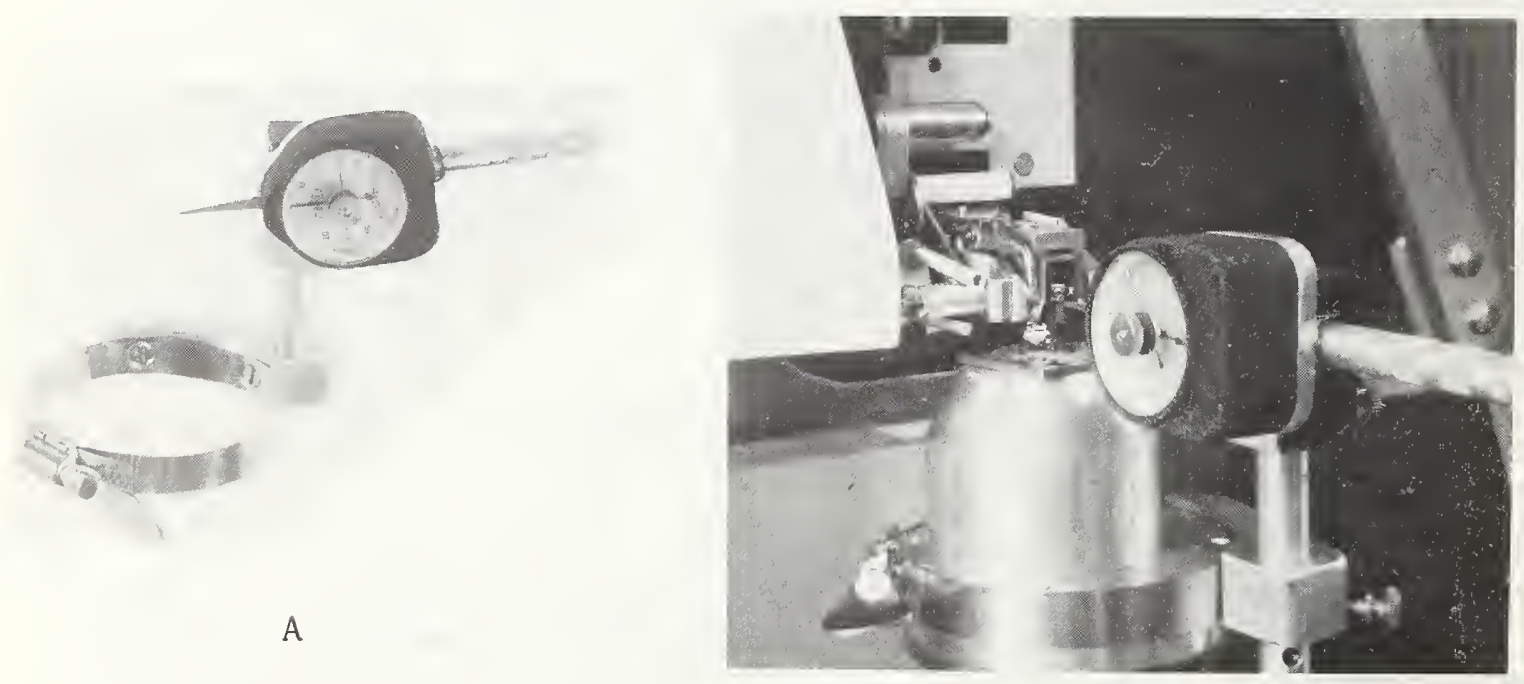

B

Fig. 1-13. Fixture used in measurement of bonding force (A), shown mounted on bonding machine (B). 
15 mils above the substrate and the indicated force is read on the gage. The same procedure should be used for the second bond position. The difference in force between the first bond and second bond readings should not be more than approximately 10 percent. If a higher value is measured, it indicates that the transducer pivot bearings are too tight. Also if the measured force changes more than 1 or 2 grams as the transducer is lifted up approximately $1 / 8 \mathrm{in.}(3 \mathrm{~mm})$ then the bearings need adjustment.

(H. K. Kessler and A. W. Stallings)

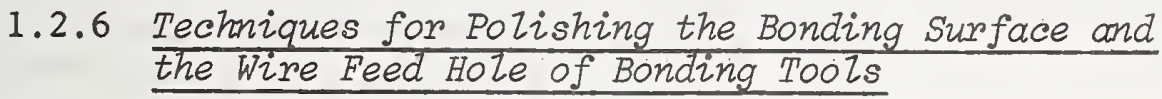

A simple and convenient technique for repolishing the bonding face of worn or pitted flat-bonding tools has been devised. This technique requires no specialized equipment. A flat copper plate loaded with a diamond-bort paste is placed on the work-stage of the bonding machine. The tool is lowered onto this polishing plate by adjusting the search-height control. Polishing is accomplished by moving the work stage under the stationary tool in a general figure-eight pattern, while the tool is applied with a force comparable with that used in bonding. During polishing, it is essential that the bonding tool be perpendicular to the diamond-loaded copper plate. The tool is removed and cleaned ultrasonically before it is used for bonding. A preliminary evaluation of the method was carried out with diamond paste commercially specified as $3-\mu \mathrm{m}$ particle size and by polishing for 1 min. This produced such a highly smooth finish that the tool had to be broken in by making a number of bonds at high power. If the tool is not worn, aluminum or gold buildup may be cleaned off simply by rubbing the tool-face with crocus cloth. This will not abrade the tungsten carbide.

The feed holes for round or ribbon wire in ultrasonic bonding tools are generally very rough and have sharp edges on the exit hole as a result of electro-etch-machining. These defects may scratch the surface of the bonding wire. Small shavings, which have occasionally been observed to come from the wire, could enter the device, causing electrical shorts. In some cases the wire may catch on the sharp points resulting in a nonuniform loop height. An inexpensive, electric vibrating engraving tool was used to polish the wire feed hole in the bonding tool. To do this a steel or bronze wire was fastened by a clamp or soldered to the engraving tool tip and coated with $3 \mu \mathrm{m}$ diamond paste. The coated wire was inserted into the wire feed hole in the bonding tool. The vibrating motion, approximately 7200 strokes per minute, polished the hole and smoothed the edges in about $30 \mathrm{~s}$. Care has to be taken to adjust the stroke of the engraver to prevent the polishing wire from damaging the heel corner of the bonding surface. This technique is also applicable to polishing the capillary hole in thermocompression bonding tools.

(H. K. Kessler) 


\subsubsection{Sintering Temperature and Bondabizity of Aluminum Metallization}

[527] An evaluation was made of substrates prepared with different sintering temperatures in order to establish conditions which yielded reproducible bond strengths, as determined by a pull test. Silicon wafers were prepared in pairs. One wafer of each pair had a $0.75-\mu \mathrm{m}$ thick layer of thermally-grown oxide while the other had none. A $0.5-\mu \mathrm{m}$ thick aluminum film was evaporated on each wafer. An array of square bonding pads was produced by standard photolithographic methods. The wafer pairs were sintered for $15 \mathrm{~min}$ at $425,500,550$, and $575^{\circ} \mathrm{C}$ in a helium atmosphere.

U1trasonic bonds were made on each specimen with 0.001-in. diameter aluminum wire at various power settings of the ultrasonic wire bonder. The single-loop wire bond strength was measured by a pull test with a simple hook at the center of the loop. By means of a one-sided t-test at a 95 percent confidence level [7], it was found that the oxidized specimens sintered at 500 and $550^{\circ} \mathrm{C}$ were statistically similar and that they both yielded higher pull strength than the oxidized specimens sintered at either 425 or $575^{\circ} \mathrm{C}$. For the unoxidized specimens, the curves were uneven and the results were inconclusive.

(K. O. Leedy, J. K. Madello, and J. Krawczyk) 


\subsection{THE ULTRASONIC BONDING MACHINE}

\subsection{Setup and Checkout Procedures}

\subsubsection{Introduction}

In order to make reproducible bonds it is necessary to first determine the optimum bonding schedule (combination of ultrasonic power, time and bonding force) for the materials being bonded. Generally such a bonding schedule is established empirically for each integrated circuit type or transistor package.

After obtaining the schedule it is essential to maintain it for as long as that type of device is being assembled. Simple quick measurements of the ultrasonic system should be carried out daily on a routine basis.

\subsubsection{Initial Procedures*}

A new ultrasonic bonding machine was acquired to be used for the statistical analysis of various bonding parameters. Initial experiments were performed to establish the optimum operating parameters for the new machine. These are the values of force, time, and ultrasonic power which yield the most repeatable pull strengths for a group of consecutively made bonds. The results of this initial work are reported to indicate the methods that are presently used to set up the bonding machine. From previous experience, acceptable force values for 1-mil (25- $\mu \mathrm{m})$ diameter aluminum wire lie between 20 and $30 \mathrm{gf}(196$ and $294 \mathrm{mN}$ ). (See Section 1.2.5 for measurement methods.) Initial experiments included four such values: $21,24,27$, and $30 \mathrm{gf}(206,235,264$, and $294 \mathrm{mN})$. At each of these force settings, four values of time were selected: $47,75,100$, and $130 \mathrm{~ms}$. For each of the combinations of force and time, bonds were made at five or six different power settings, from the lowest to the highest power setting that is possible to make a bond. At a power level that is too low, the wire does not stick to the pad. At too high a power level, the bond is so greatly deformed that it is severed at the heel during bonding. The reported data lie within these two extremes.

Bonds were made in groups of 10 at each of the different combinations of force, time, and power for the first bond and then repeated separately for the second bond. Bonds were tested by pulling to destruction. In order that the first bond be tested independently of the second, epoxy was placed over the second bonds of the bond loops to prevent breakage at that point. The first bonds were similarly epoxied when testing the second bonds. In all, 186 groups of 10 bonds each were tested.

See also Section 4.5, p. 94 
The best results obtained for first and second bonds are shown in Figs. 2-1 and 2-2, respectively. In each case the bonding force was $27 \mathrm{gf}$ (264 $\mathrm{mN}$ ); the bonding time was $47 \mathrm{~ms}$ for the first bond and $100 \mathrm{~ms}$ for the second bond. Each point on the graph represents the average pull strength for 10 bonds made on a single level with a ratio of bond separation to loop height, $d / h$, equal to 2.5 . The measured pull strength should be multiplied by approximately 0.8 to give the actual force in the wire at breakage. In general, the shape of the curves for the first and second bonds differed, particularly in the low-power regions. The pull strength of the second bond drops to a lower value before lift-off occurs than is the case for the first bond. In the high-power region, however, the pul1 strength for the first bond falls off more rapid1y than that of the second bond. The reasons for this behavior are not fully understood but may be related to the crack that exists in the heel of the first bond. The error bars in both figures, which represent the 95 percent confidence interval for the mean, are at least twice as large as desired, apparently resulting from problems with the wire clamp and loop height control.

The next step in the optimizing procedure is to select a specific force setting and choose reduced power and time ranges based on the data obtained. The experiment is then repeated and further reduced ranges are selected until the single best operating point is obtained. It should be noted that the values of the bonding machine parameters chosen as optimum are not selected on the basis of the highest pull strength but rather on the basis of those settirigs that resulted in bonds with the most reproducible pull strengths. This procedure should yield sample standard deviations $\leqslant 1 \mathrm{gf}$.

(K. O. Leedy and C. A. Main)

\subsubsection{Daily Check Out Procedure}

[560] A check-out procedure for the bonding machine has been established and is implemented each work-day morning. The ultrasonic power supply is tuned for the desired vibration amplitude of the bonding tool tip using a magnetic detector (section 1.1.2). Five bond pairs are made and pulled to destruction. The pull strength results are recorded, and the average value and sample standard deviation for the set are determined. Over a period $c$ three weeks, the average wire bond pull strength was 8.9 grams forcet ( 87 $\mathrm{mN}$ ). The sample standard deviation varied between 0.25 and 0.85 grams for

$\dagger$ The selection of five bond pairs per group is based on statistical considerations regarding the estimation of the mean of a population from a single sample (see M. G. Natrella, Experimental Statistics, NBS Handbook 91, August 1, 1963, pp 2-10).

It should be noted that these bonds are single-level bonds made under ideal conditions and that they are neither annealed nor environmentall) tested before being pulled. 


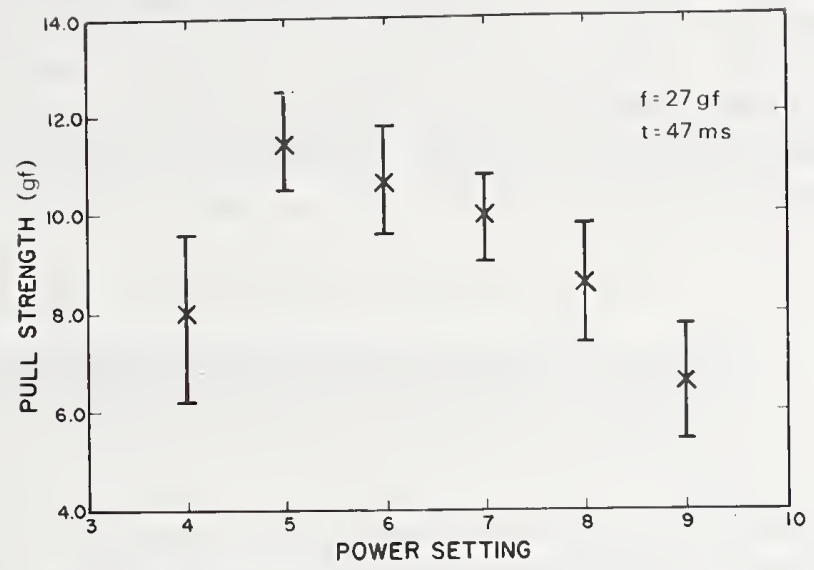

Fig. 2-1. Measured bond pull strength for a first bond as a function of machine power setting.

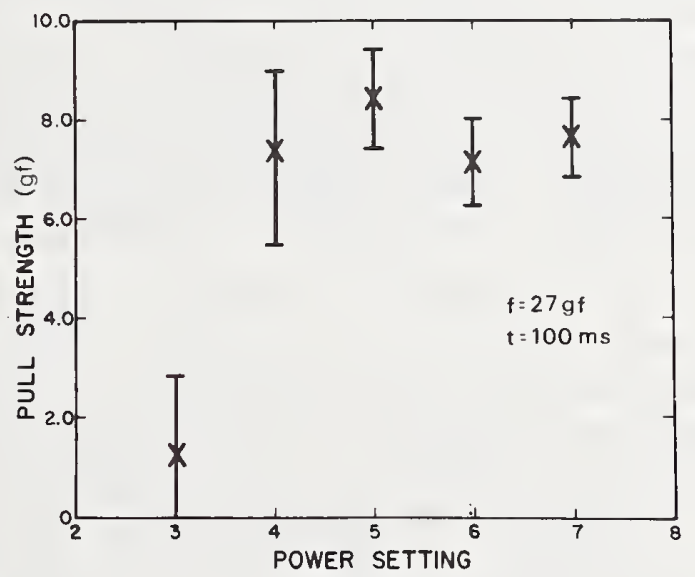

Fig. 2-2. Measured bond pull strength for a second bond as a function of machine power setting. 
(2.4, and $8.3 \mathrm{mN}$ ). The standard deviation is of particular interest since the small value indicates that the bonding process is carried out reproducibly. Further evidence of the reproducibility is the fact that the mode of failure is always a break at the heel of the first bond. These results support the conclusion that the machine parameters and other variables are under sufficient control that bonds made on this bonding machine with the chosen operating conditions are satisfactory for use in studies of the pull test.

(K. O. Leedy)

\subsubsection{Tune-up Procedure and Reestablishment of Vibration Amplitude: General Trouble-Shooting Methods*}

[573] [n order to use a capacitor microphone or magnetic pickup for tuning and trouble-shooting an ultrasonic wire bonding machine, a suitable holding fixture must be used. Such fixtures were shown in Fig. 1-4 and 1-5. Many other designs are possible. In general, however, a fixture of the above type is advantageous because, as will be discussed in section 2.2.1, it permits measurements of mechanical looseness and spurious motion of the transducer mount with respect to the work-stage that holds the device to be bonded.

The following procedure should be considered as a general guideline for tuning ultrasonic bonding machines. Variations of this procedure may be desirable for tuning particular machines or in special circumstances. It is desirable to keep day-to-day records of bonding parameters as a means of determining long-term frequency and amplitude drift from the desired bonding schedule. For maximum reproducibility, microphone measurements must be made each time with the bonding machine in the same part of its cycle, usually the "start" or "reset" position. The entire procedure should take only a minute or so longer than is normally required to change a bonding tool and retune the ultrasonic system.

(1) Position the microphone or magnetic pickup (detector) on the bonding machine as was shown in Fig. 1-4b. Adjust the tip of the detector slightly above the bottom of the bonding too 1 as shown in Fig. 1-3. After an appropriate warm-up time, turn on the test switch of the ultrasonic power supply and hold it on for a short time (preferably not over one or two minutes) with, if necessary, a rubber band or other mechanical means. Advance the detector tip until it lightly touches the vibrating bonding needle and "hash" appears on the oscilloscope. Then back it off until it just barely clears the needle. The "hash" disappears and a clear $60 \mathrm{kHz}$ sine wave signal is seen. The microphone is then adjusted upward or downward for maximum amplitude.

* This section was extracted from Tech. Note 573 and that publication (available on request) should be consulted for more details. 
(2) Observe and record the $60 \mathrm{kHz}$ sine-wave amplitude as read from an oscilloscope. This is a very important step that permits the reestablishment of this amplitude at the end of the tune-up procedure.

(3) If the bonding tool is to be changed or adjusted, do so at this time. Move the detector to the side without changing its vertical height and remove the old tool. Insert the new tool to the measured extension and tighten the set screw with a torque wrench to its recommended torque (20 to 25 in-oz for a \#2 screw).

(4) Reposition the detector as in (1).

(5) Peak tune the frequency on the power supply to produce maximum vibration amplitude of the tool. (It is preferred that an oscilloscope be used as the indicator rather than a meter, so that the output waveshape can be carefully observed for asymmetries.) Turn the power control from zero to maximum on the scale (Hi or Lo) normally used in bonding. If any waveshape asymmetries exist or if the amplitude does not increase linearly, tighten the set screw to its recommended torque, replace the tool, or both. If the waveshape still does not return to a pure sine wave or the ultrasonic amplitude does not increase approximately linearly with power, then there is a problem in the power supply, the transducer, or its mount. The latter condition often can be improved by tightening the mount screw, slightly moving the position of the transducer in its mount, etc. (see discussion below).

(6) After tuning according to step (5) if the measured vibration amplitude does not return to within 10 or 15 percent of the value recorded in step (2), then readjust the power control to return the amplitude to this value. This is the most important single step in these instructions! The bonding machine is now ready to resume the same bonding schedule established previous to step (1).

(7) The transducer is temperature sensitive; thus if a high intensity illuminator burns out or if there are other reasons to suspect significant temperature change, tune the system as above when the new equilibrium has been established.

Several ultrasonic power supply manufacturers specify meter tuning of their equipment at much higher power levels than are generally used for wire bonding. In many cases the power supply meter will not indicate the peak mechanical resonance of the transducer. Even if it does, the resonance frequency may be power sensitive, and the unit will not be properly tuned when it is used at normal power levels. This situation can be quickly diagnosed with a microphone or magnetic pickup. First tune the bonder at the specified conditions. Then lower the power to that of the normal bonding range and retune according to step (5) above. If the lower-power resonant frequency is different from the higher power one, 
then a transducer-tool interaction, a transducer-mount interaction, or most probably a faulty transducer is indicated. The tool should be changed to eliminate the possibility of transducer-tool problems. After this, a verification of these problems can be obtained by measuring the detector output versus power-dial-control setting of the ultrasonic power supply. The curve should be approximately linear. If it saturates, decreases at high power, or requires continual peak tuning, then transducer and/or mount problems exist.

A further indication of trouble can frequently be seen by a nonlinearity in the oscilloscope wave shape display of the microphone output during the linearity test or after changing the tool. An illustrated discussion of this problem is given in section 2.3.6. In general, all problems that cause non-linear wave forms increase in severity as the power is increased. A bonding machine-transducer combination may be linear and perform well for bonding 1 mil wire (< 1 watt of power), but show severe distortion, tool amplitude loading, etc., when used to bond thick wire at $\sim 5$ watts or greater. A transducer-mount interaction can often be corrected by tightening the mount screw and/or by adjusting the transducer slightly backward or forward in its mount. If this does not help, then the problem is most likely in the transducer, which should be changed. The transducer mounts on some older bonding machines may have been inadequately designed and even changing the transducer will not help.

With some bonding machines, large bond deformations ( 2-3 wire diameters) may occur repeatedly on the first and/or second bond even when the ultrasonic power is reduced. Such deformations may be attributed to an ultrasonic system malfunction. However, the condition more often results from mechanical problems, such as worn or nicked cams, broken or poorly adjusted transducer bearings, excessive bonding force, or any other mechanical condition that will cause too high an initial impact force of the tool against the wire. To diagnose this condition a series of bonds should be made with progressively decreasing ultrasonic power. The deformation should decrease. The impact deformation of the wire with zero ultrasonic power should be < 1.5 wire diameters. If larger, then one or more of the above mentioned mechanical problems is indicated, rather than the ultrasonic system.

(G. G. Harman and H, K. Kessler) 


\subsection{Some Mechanical Problems of Bonding Machines}

\subsubsection{Low Frequency Motion and Bond Formation}

Low-frequency differential-motion between the bonding tool and the bonding pad during ultrasonic bonding is a major cause of unreliable bonds. Such motion can originate from within the bonding machine due to the torque of its programming motor and cams, from various forms of operator movement, or from external motion such as building vibration. On assembly lines, often as many as 10 bonders are placed close together on a single long table. Vibrations originating from one machine or operator may interfere with bonds made on adjacent machines. Bonders are frequently installed on upper floors of buildings and/or close to heavy machinery or both. Vibrations can often be felt by merely touching a table or wall. This becomes meaningful when one realizes that any vibrations which can be sensed in this manner represent motions in the order of 1 mil $(25 \mathrm{\mu m})$, and that wave cancellation and reinforcements can result in much of the vibration amplitude appearing as differential motion between the bonding tool and the device. This can seriously affect the quality of ultrasonic bonds made with small. diameter wire ( $~ 1$ mil).

The conclusions to be drawn from the following work are: (1) Extraneous motion is the major cause of bond to bond deformation variations and a significant cause of unreliable bonds; (2) the operator should be cautioned about the detrimental effect that her own movements can have on bond formation; (3) each bonding machine should be placed on a separate table; (4) bonding assembly lines should be located in a vibration free environment, preferably on a ground-level cement-slab floor.

Capacitor-microphone displacement measurements revealed a lack of mechanical rigidity in some bonding machines (NBS Tech. Note 527, p. 39). This results in undesired movement of the bonding tool, held by the transducer, with respect to the transistor or integrated circuit, held by the work stage. Such movement can be transmitted to the machine in a number of ways, including motion of the bonding operator's arm as it rests on the machine, motion of the work stage as a result of loose mechanical tolerances due to poor design or wear, or operator movement if the work stage is rotated by hand. In the last case it has been observed that a slight twitch of the operator's hand can result in work stage movement on a typical machine of greater than 0.001 in. $(25 \mu \mathrm{m})$. With respect to machine instability, the torque of the programming cam motor may produce detrimental vibrations. If any of these occur during the actual bonding period, a lift-off or low pull-strength bond may result. (G. G. Harman) 


\subsubsection{Motion Perpendicular to the Wire}

[555] Experiments were conducted in which work-stage motion was intentionally introduced during the bonding period to characterize the effects of such motion. Sideway motion was introduced by mechanically driving the work stage with an electro-mechanical transducer at various frequencies and displacements. Bonds were observed visually and with the scanning electron microscope. As the degree of motion increased, the bond deformation began to vary widely from bond to bond and an increasingly greater number of lift-off bonds were produced. With motion in the order of 0.001 in. (25 $\mu \mathrm{m})$ more than half of the bonds lifted off.

Examples of lift-off patterns for bonds made while the work stage had an intentional sideways motion of less than 0.0005 in. $(13 \mu \mathrm{m})$ are shown in Fig. 2-3. This amount of motion has been frequently observed in typical bonding machine installations on commercial production lines. While the effects of the motion are clearly apparent when the pattern is examined with a scanning electron microscope, they would be difficult to recognize from examination with an optical microscope.

(K. O. Leedy and G. G. Harman)

\subsubsection{Motion Paralzel to the Wire}

[571] Motion was introduced parallel to the wire direction and ultrasonic tool motion at $20 \mathrm{~Hz}$ to simulate building vibrations and $60 \mathrm{~Hz}$ to simulate electrical equipment vibration.

The relative positions of the wire and tool during formation of the first bond are indicated in Fig. 2-4. It is in this position that the normally sharp heel of the tool deforms the wire during bonding

shape. The greatest effect of the front-to-back motion is on the heel shape; increased motion results in more deformation and cracking. The extent of this depends on the radius of the tool heel. Three tools with different heel radii were used in the test. One had a sharp heel with no intentional radius; heel radii of the others were specified as 0.2 and $1.0 \mathrm{mil}$ (5 and $13 \mu \mathrm{m}$ ). Motion amplitudes of approximately $0.2,0.4$ and one $\mathrm{mil}(5 \mu \mathrm{m}, 10 \mu \mathrm{m}$, and $25 \mu \mathrm{m})$ were used at both the $20-\mathrm{Hz}$ and $60-\mathrm{Hz}$ driving frequencies. SEM photomicrographs of typical bonds made under these conditions are shown in Fig. 2-5. A bond made with the sharp-heeled tool under ordinary conditions without motion is shown in Fig. 2-5a.

There is only a small crack in the heel; both other tools produced similar bonds under this condition. For $0.2 \mathrm{mil}$ motion at $20 \mathrm{~Hz}$, the crack in the heel was slightly enlarged, but the actual shape of the bond was not greatly different from the case of no motion. For 10-um motion at $20 \mathrm{~Hz}$, the sharp-heeled tool almost severed the wire from the bond as shown in Fig. 2-5b. Increasing the motion to one mil often caused the wire to break. For the tool with a $0.2 \mathrm{mil}$ heel radius less heel damage occurred. Although the heel was thinned, cracking was not severe. The deformation of the wire increased with motion, often by a factor of two in going from 


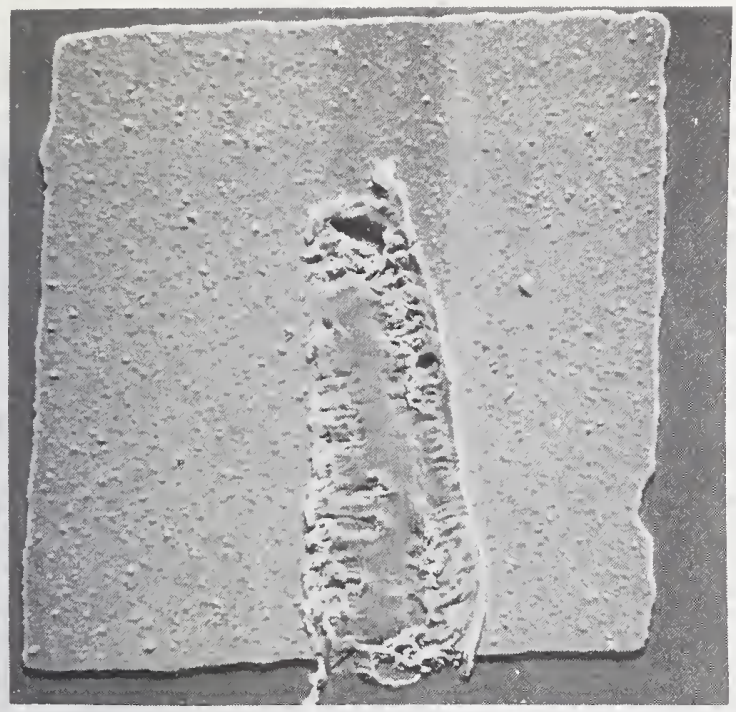

a: Magnification: $460 \mathrm{x}$.

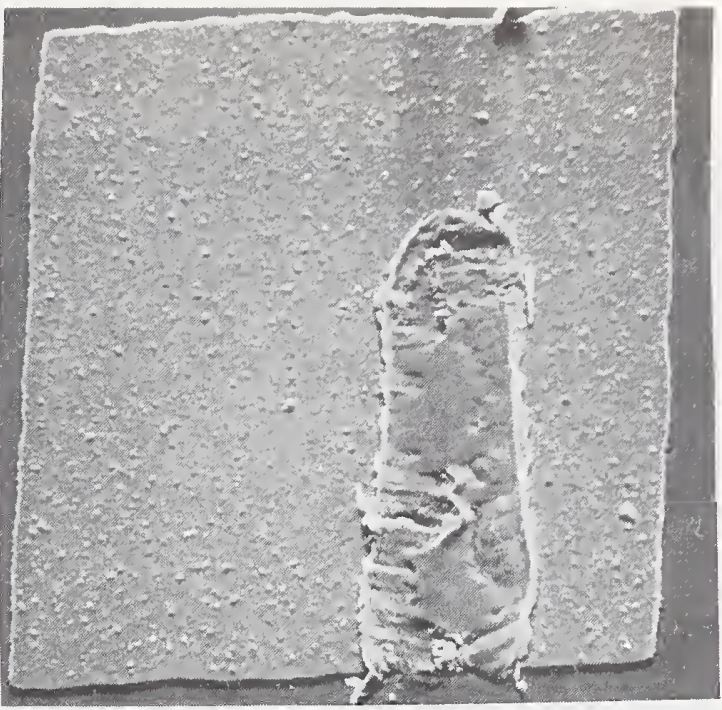

b: Magnification: $475 \mathrm{x}$.

Fig. 2-3. SEM photomicrographs of lift-off patterns of bonds made with moderate $(<0.0005$ in.) sideways motion of the work stage during bonding. In both cases, some sideways tearing of the bond is evident. Additional evidence of motion in case $\mathrm{a}$ is the incomplete wire-to-metallization imprint on the left side of the pattern, and in case $b$, the smoothing of a portion of the normally rough weld surface.

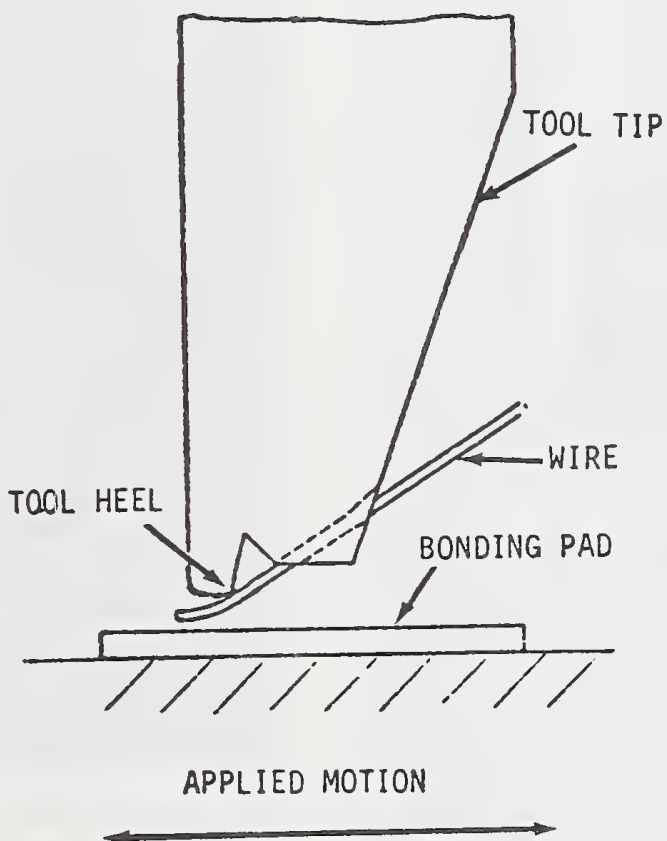

Fig. 2-4. Relative positions of wire and bonding tool tip just prior to formation of the first bond. 


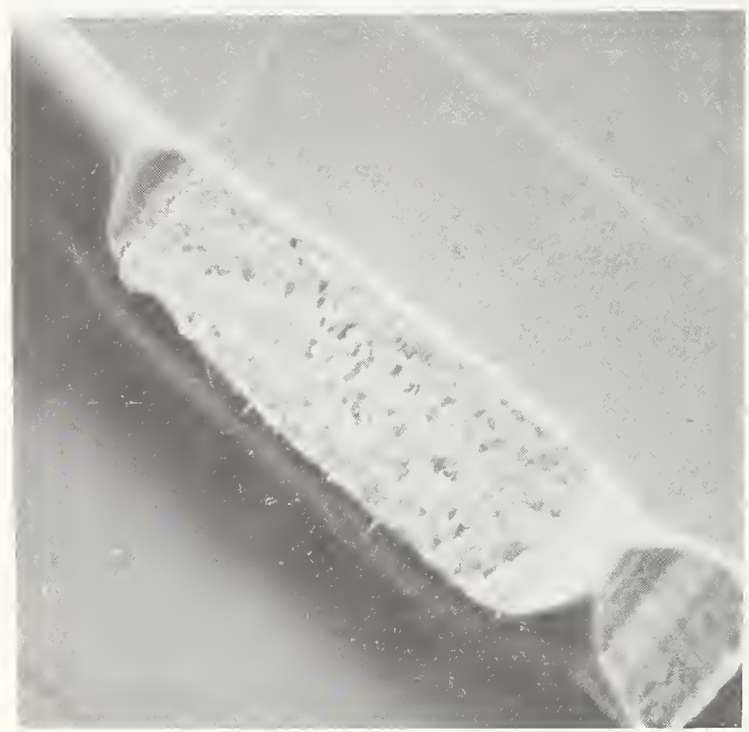

a: No motion; Magnification $650 \mathrm{x}$.

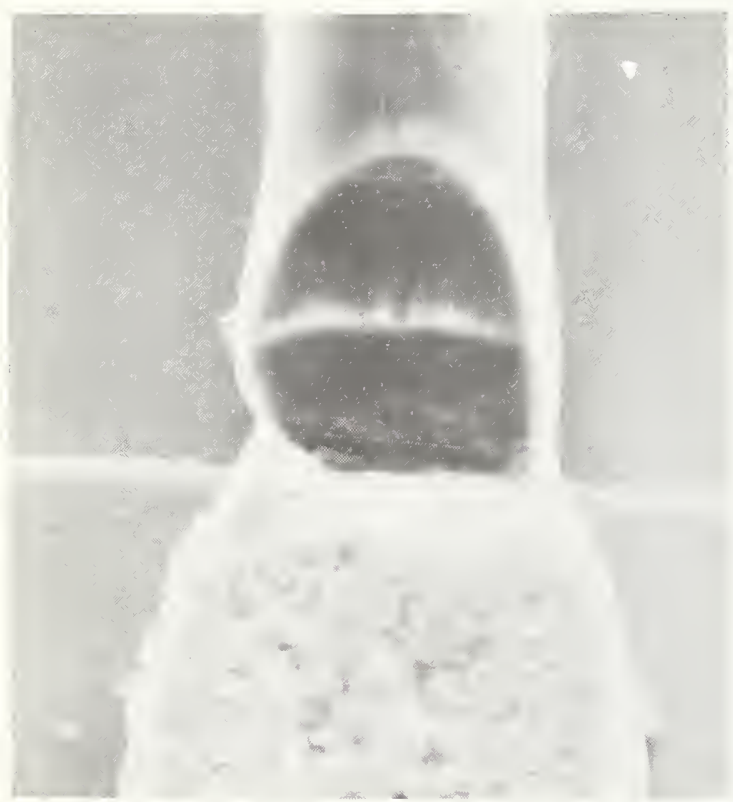

b: Amplitude: $10 \mu \mathrm{m}$; Frequency: $20 \mathrm{~Hz}$; Magnification: $1100 \mathrm{x}$.

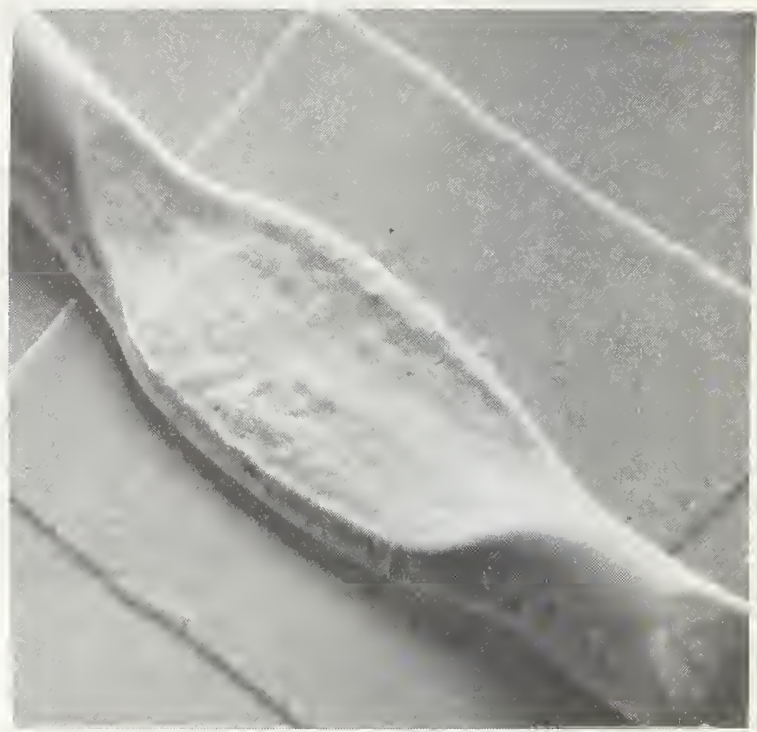

c: Amplitude: $~ 25 \mu \mathrm{m}$; Frequency: $20 \mathrm{~Hz}$; Magnification: $600 \mathrm{x}$.

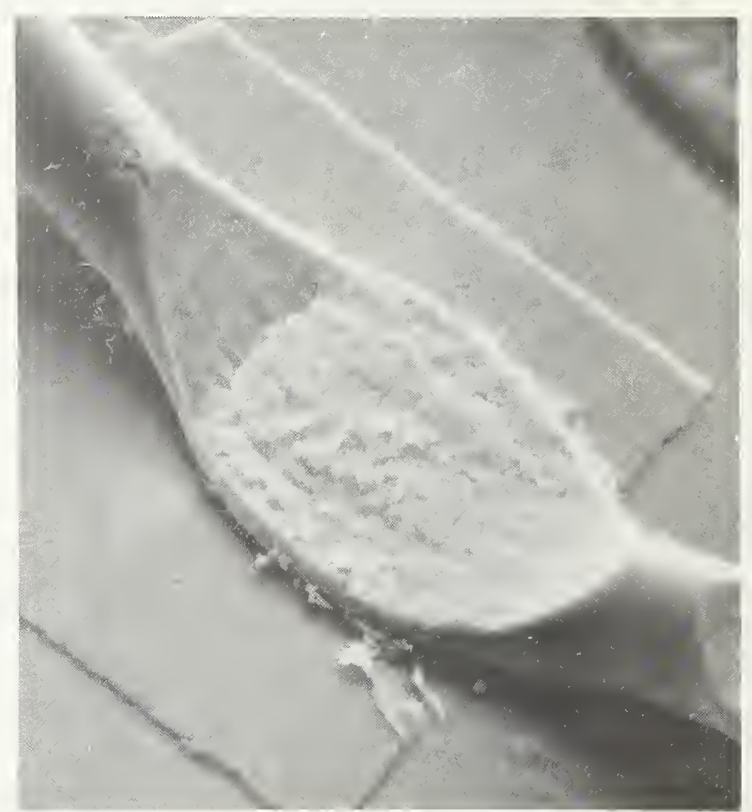

d: Amplitude: $10 \mu \mathrm{m}$; Frequency: $60 \mathrm{~Hz}$; Magnification: $550 \mathrm{x}$.

Fig. 2-5. SEM photomicrographs of typical first-bonds made with various relative front-to-back motion between bonding tool and work stage. 
no motion to one mil motion, but the wire was not cut. An example of a bond made with the $0.2 \mathrm{mil}$ heel radius and one mil motion at $20 \mathrm{~Hz}$ is shown in Fig. 2-5c.

Motion at $60 \mathrm{~Hz}$ produced similar results but the deformation was increased. For example, $0.2 \mathrm{mil}$ motion at $60 \mathrm{~Hz}$ caused the bond to have an appearance similar to that of one made with $0.4 \mathrm{mil}$ motion at $20 \mathrm{~Hz}$. An example of a bond made with the $0.5 \mathrm{mil}$ heel radius and $0.4 \mathrm{mil}$ motion at $60 \mathrm{~Hz}$, shown in Fig. 2-5d, illustrates the thinning at the heel that accompanies the increased deformation. As expected, typical bond shapes for bonds made with the tool with a $0.2 \mathrm{mil}$ heel radius under the different motion conditions were between the two extremes just described.

Two catastrophic failure modes due to parallel motion are illustrated in Fig. 2-6. Actual severance of the wire due to cutting by the sharp heel is shown in Fig. 2-6a. Lift-off which occurs when the motion has broken the weld at the wire-metallization interface is shown in Fig. 2-6b. The type of failure mode depends on the portion of the bonding cycle in which the maximum motion occurs. Higher frequency motion tends to produce greater but more reproducible damage at lower displacements, since several cycles of motion may be integrated over the bonding period.

(K. O. Leedy and G. G. Harman)

\subsubsection{Self-Induced Motion in Bonding Machines}

An electromagnetic displacement sensor (described in NBS Tech. Note 560, p. 37) was used to seek the source of the extraneous self induced motions which have been observed in bonding machines. In this application of the sensor, part of the detector is mounted on the machine housing and the other part is clamped to the work stage.

The most severe motions were found in the side-to-side and vertical directions. In the side-to-side direction, the upper part of the bonding machine moved in relation to the work stage during both the first and second bond cycles. A support bracket was made and mounted between upper and lower bonder parts to decrease the movement. In addition to the movement, a vibration, which results principally from the starting torque of the stepping motor and camshaft assembly, was observed. In the vertical direction, no movement such as that observed in the side-to-side direction is seen.

A typical oscillogram of the motion between the machine housing, which supports the bonding tool, and the work stage with the motor and all cams operating is shown in Fig. 2-7. The indicated motion is in the side-toside direction. The trace in Fig. 2-7a covers the part of the bonding cycle from first search through bonding to loop formation. The upper trace displays the ultrasonic bonding pulse to indicate when the bonding occurs. The lower curve, on the same time scale, shows the various selfinduced bonding machine vibrations. Maximum vibration amplitudes of 


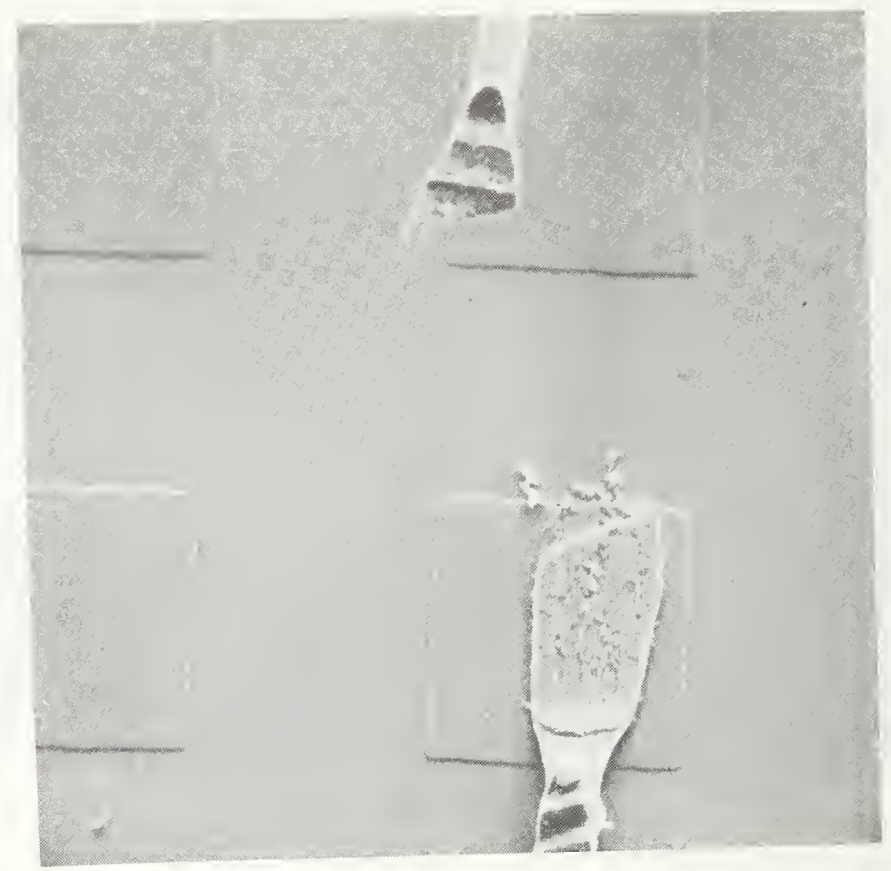

a. Severance of wire by heel of tool. Magnification: $200 x$.

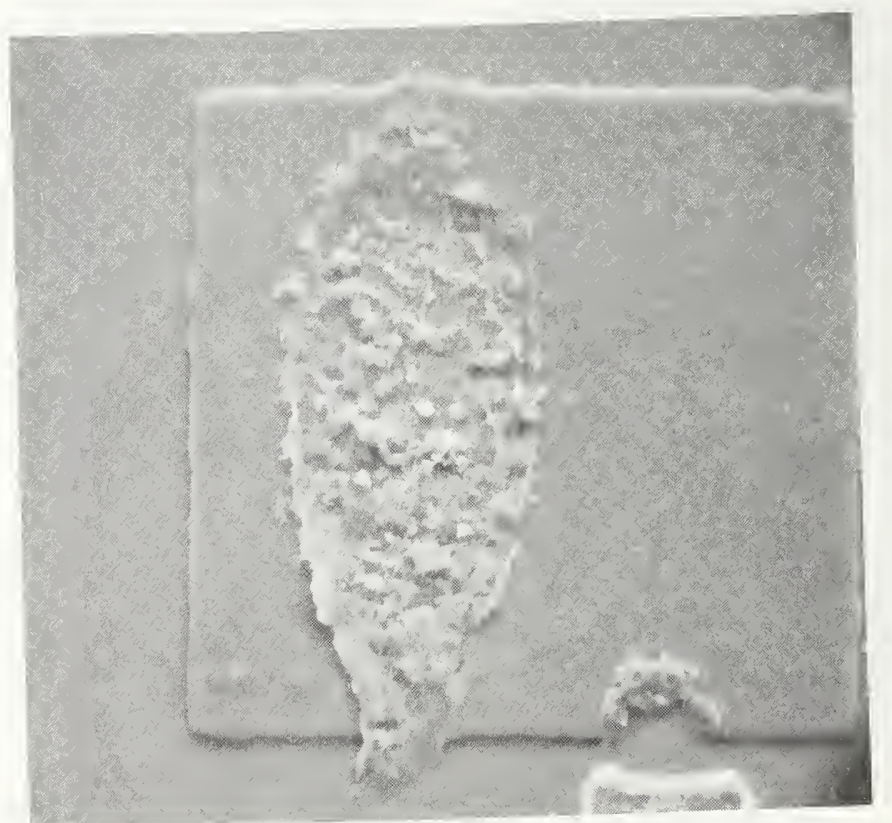

b. Lift-off at weakened weld. Magnification: $500 \times$.

Fig. 2-6. SEM photomicrographs of catastrophic failure modes that result from front-to-back motion. 


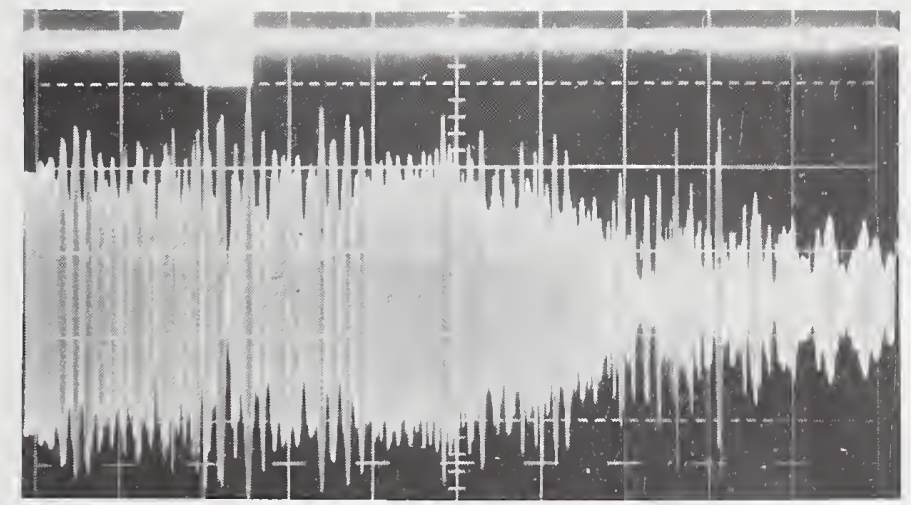

a: First search position through bonding and into loop position.

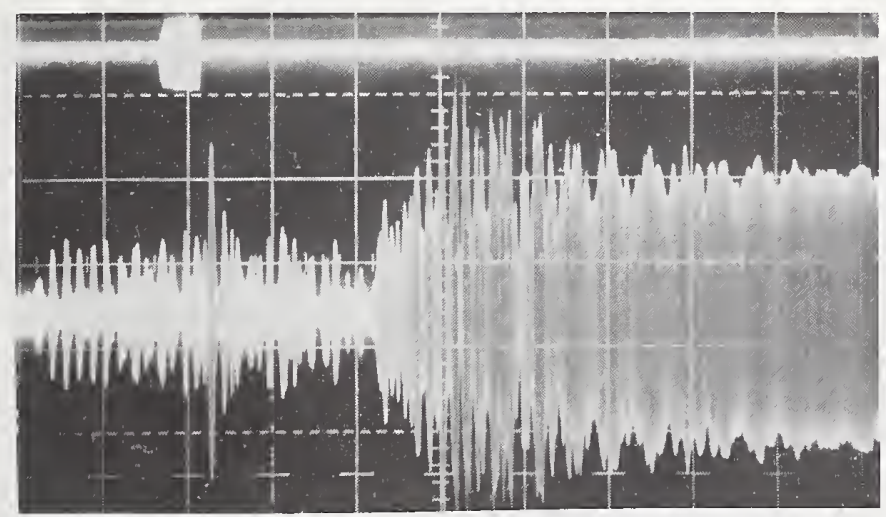

b: Second search position through bonding and wire cutoff to reset position.

Fig. 2-7. Measurement of the self-induced vibrations of a bonding machine. The upper trace displays the ultrasonic bonding pulse. The lower trace gives the output of the displacement sensor. The vertical scale for the lower trace (displacement) is $2.5 \mu \mathrm{m} / \mathrm{div}$. The horizontal scale for both traces is $100 \mathrm{~ms} / \mathrm{div}$. 
about $0.5 \mathrm{mil}(13 \mathrm{\mu m})$ peak-to-peak that occur during and immediately after the bond is formed are indicated by fluctuations in the envelope amplitude.

The trace in Fig. 2-7b covers the next part of the bonding cycle from second search, through second bonding and wire cutoff, to the start or reset position. A temporary displacement of the housing which occurred earlier in the cycle continued until wire cutoff, at which time the housing moved back to its original position accompanied by large vibrations with an initial amplitude approaching $0.5 \mathrm{mil}$. After this, the vibrations damped out as the machine reached its starting position and the cam motor stopped.

A vibration study was made of each individual cam, including the operating levers, in order to determine in which cam and lever combination the vibration originated. It was found that each cam and lever has its own vibration characteristics, but if all cams and levers are operating, some vibrations are amplified and others are cancelled out.

(H. K. Kessler)

\subsubsection{Wire Clomp Problems}

[527] Bonds from several devices selected from the output of operational production lines were examined with the scanning electron microscope (SEM).

The crack at the bond heel shown in Fig. 2-8a is a feature of all first bonds made on these production lines. In addition, deep indentations were observed on the sides of the wire. It has been determined by additional SEM photographs that these marks are produced by the jaws of the clamping system through which the wire is fed from the spool to the bonding tool. In addition to marring the surface finish, these imprints could work-harden the wire and thus affect bonding conditions. Occasionally the wire twists after passing through the clamping system, and the indented portion then becomes a part of the actual bond interface as shown in Fig. 2-8b. Of particular concern is the possibility of contamination from the clamp becoming embedded in the wire and directly interfering with the bonding process. Since the twists, such as those depicted in Fig. 2-8b, occur only occasionally and since not all of these need be significantly contaminated, the possibility should be considered that this may be a cause of occasional, otherwise unexplained weak bonds. One solution to this problem is to use large, 2 inch diameter, wire spools instead of the more usual $1 / 2$ inch spools. Wire from the large spools shows little tendency to twist. Also the wire clamps should be polished and the clamping force should be minimized.

(K. O. Leedy) 


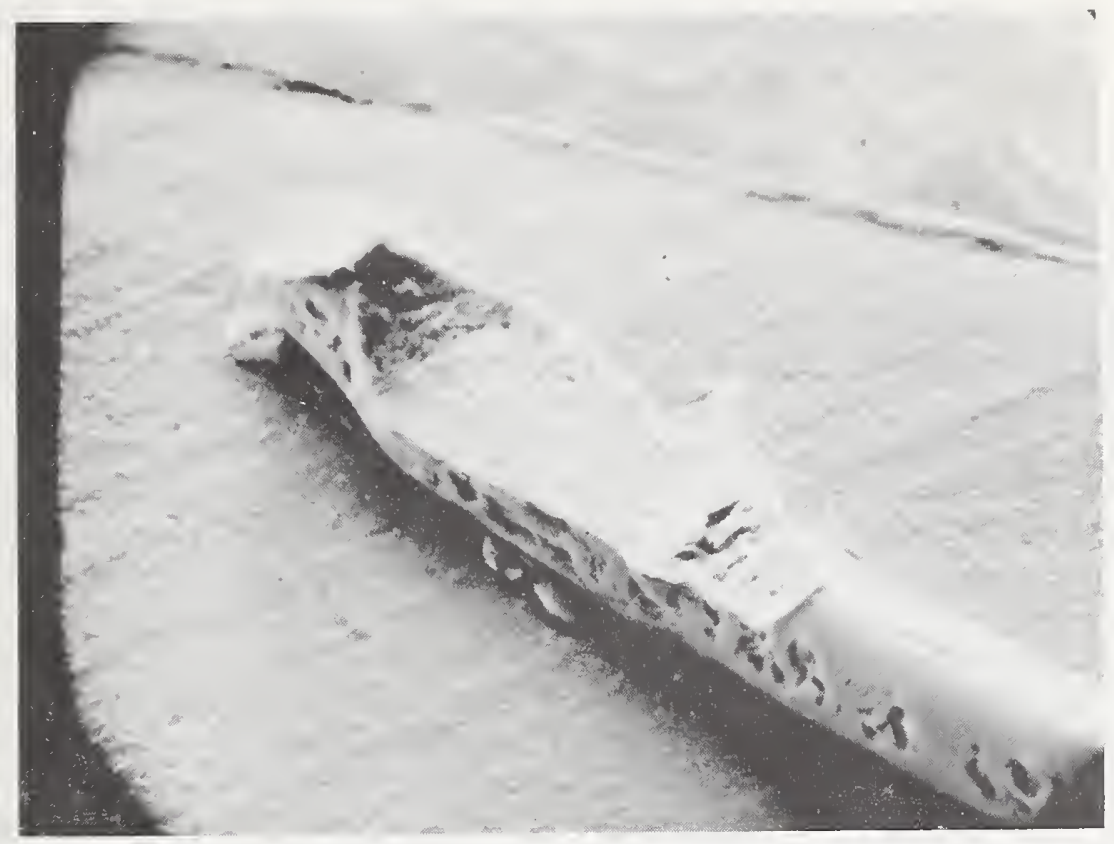

a: Normal clamp mark configuration.

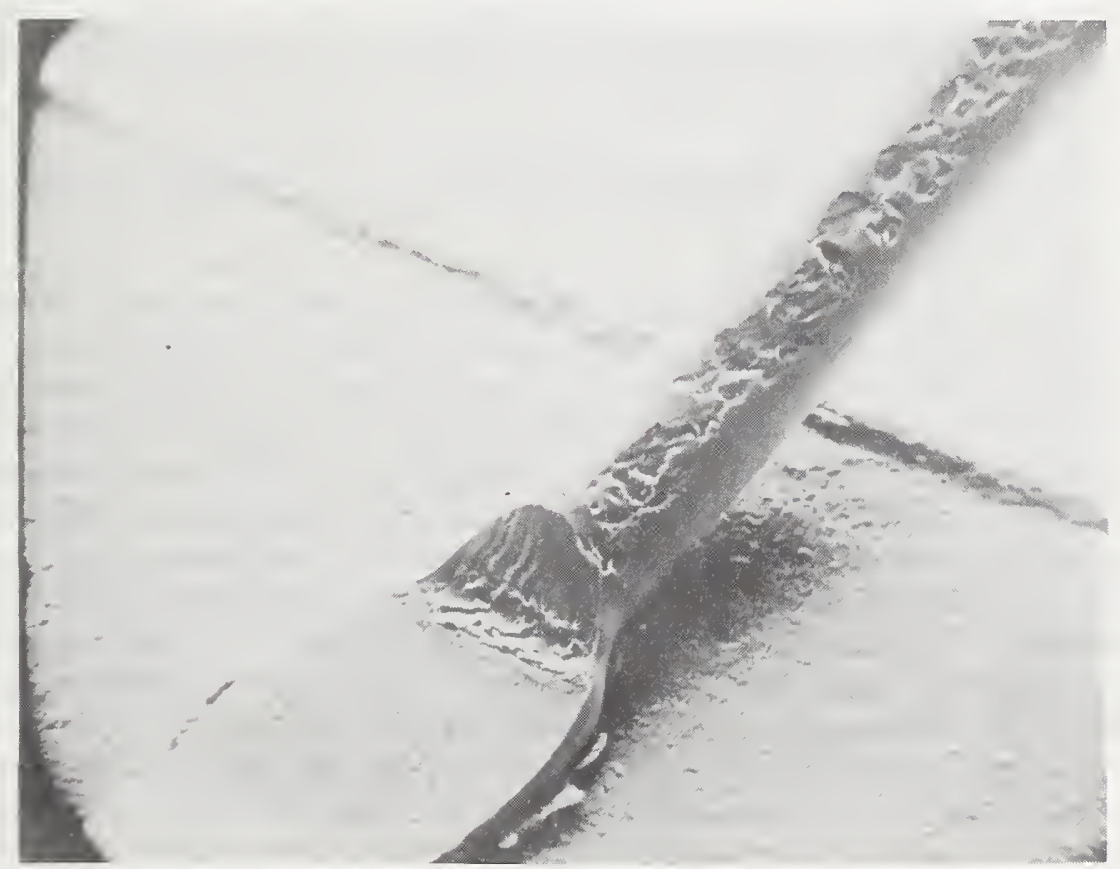

b: Wire has twisted after being clamped and before bonding so that the clamp marks are incorporated in the weld interfacial region.

Fig. 2-8. SEM photomicrographs (575X) of aluminum ultrasonic wire bonds showing heel crack and clamp marks. The clamp marks appear on both sides of the wire. 


\subsubsection{Temperature Characteristics of Transducers}

[520] Under some conditions, ambient temperature variations may detune the reson ant frequency of transducers. Therefore, a study was made to determine the temperature dependence of transducers having both ferrite and nickel magnetostrictive ultrasonic elements. A linear temperature coefficient of 17 to $20 \mathrm{~Hz} /{ }^{\circ} \mathrm{C}$ over a range of 18 to $30^{\circ} \mathrm{C}$ was measured for both types of transducers. This value is consistent with the expansion characteristics of the stainless steel parts and is satisfactorily low for normal bonding machine use. However, some modern bonding machines have multiple high intensity lamps that illuminate an extended area around the work. Since these lamps are capable of heating the transducer by 5 or $10^{\circ} \mathrm{C}$, changes in the illumination can cause measurable changes in the operating characteristics of the transducer if the ultrasonic system $Q$ is greater than approximately 100. As the transducer warms up, the resonant frequency will drift downward 100 to $200 \mathrm{~Hz}$. Thus to obtain reproducible bonding conditions with a particular ultrasonic system having a $Q$ of

[527] $\approx 135$ (see Fig. 2-19c) it was necessary to preheat the transducer by turning the lights on for several hours prior to bonding, or to put heat absorbing filters in front of the lamps.

(G. G. Harman)

\subsubsection{Transducer Efficiency Variations Observed on Assembly Lines}

[598] Four visits were made to semiconductor device producers at the request of sponsors. Calibrated microphones and magnetic pickups were distributed along with copies of instructions for their use (NBS Tech. Note 573). Measurements were made of the ultrasonic vibration amplitude of the bonding machines at each site. Even though all lines had identical equipment, significant variations in tool tip vibration amplitude and bonding time were found. Twenty-five percent variations were common. However, on one production line, a factor of two variation in power supply power dial settings was observed to produce the same tool tip vibration amplitude from one bonding machine to the next. This is attributed to variations in transducer conversion efficiency rather than to properties of the power supply. In another case, involving the least efficient transducer, the technician had apparently been unwilling to increase the power dial setting sufficiently to compensate for the transducer inefficiency; instead he increased the bonding time in order to establish good bonding. As it tumed out, that machine produced the most reliable bonds on that assembly line, so it would appear that an optimum bonding schedule had been fortuitously achieved on this one machine. Although the intuition of a good technician is valuable in setting up a bonding machine, only by actual measurement of the tool vibration amplitude is it possible to set up a known bonding schedule on a number of apparently identical bonding machines.

(G. G. Harman and K. K. Kessler) 


\subsubsection{Laboratory Setup for Measuring Transducer}

In order to accurately study the vibration modes of bonding tools in various experiments it was necessary to design a mount that would rigidly hold both the microphone and the transducer, but allow controlled vertical and horizontal displacement as required. The apparatus is shown in Fig. 2-9. In addition to micrometer-controlled translational movement along three perpendicular axes, the microphone can be rotated $\pm 135 \mathrm{deg}$. around the tool in order to observe sideways and other modes of vibration.

\subsubsection{Bonding Tool Vibration Modes}

Investigation of the unloaded vibration amplitude along the entire tool length has shown that the tool extension above the horn can significantly affect the resonant frequency and other characteristics of the transducer-tool system. The data shown in Fig. 2-10a were taken from the same tool, driven under the same conditions as were used to obtain the data for curve c of Fig. 2-19. In this case the residual extension above the top of the horn is approximately a resonant length. Under similar conditions, different bonding tools have been observed to have amplitude maxima above the horn greater than ten times those below the horn. Since the normal specified tool tolerance is $\div 0.015$ in.* $(0.38 \mathrm{~mm})$ and since only the tool extension below the horn is measured when mounting a new tool, it is possible that the residual extension above the horn can vary as much as 0.03 in. $(0.76 \mathrm{~mm})$ from tool to tool. When the measurement was repeated with a different tool, $0.013 \mathrm{in} .(0.033 \mathrm{~mm})$ longer than the one used in the measurement shown in Fig. 2-10a, the amplitude of the top portion was similar to that of the bottom. The changes in system resonant frequency and mechanical $Q$ which result from large resonances at the top of the tool require significant adjustment of the ultrasonic power supply power-control-setting to reestablish the original bonding tool motion. It was also observed that, when the maximum above the horn was as much as ten times that below, these parameters become critically dependent on the tightening torque applied to the bonding tool set screw. Fig. 2-10b gives the unloaded vibration modal pattern for a short bonding tool.

(G. G. Harman)

* All except one of the tools used in this study were well within the specification limits. This suggests that tool length tolerances can be tightened considerably. 


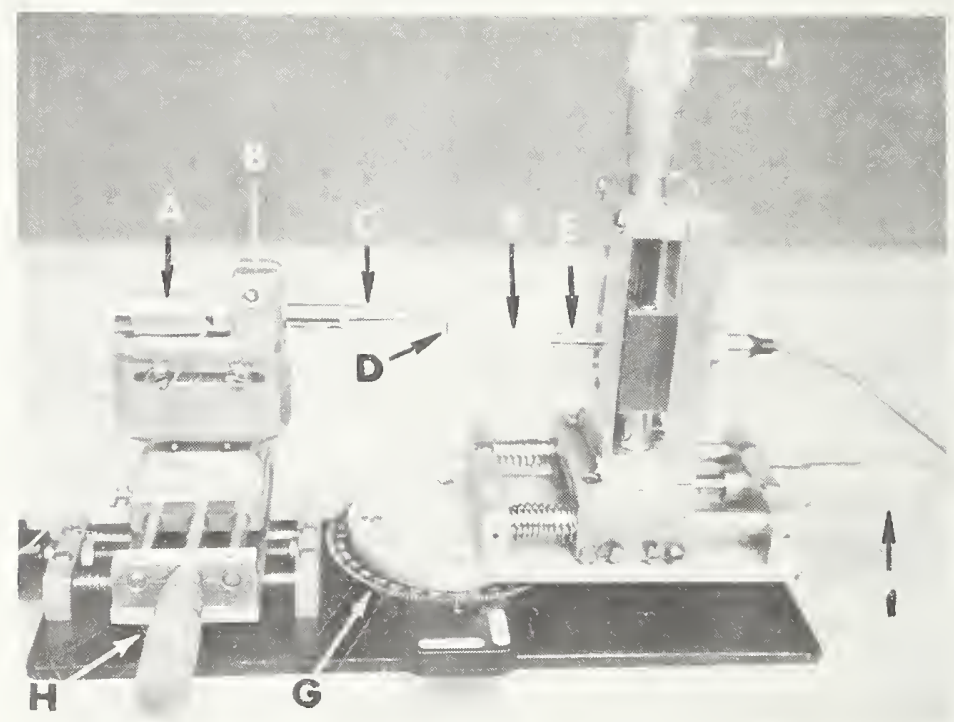

Fig. 2-9. Precision ultrasonic transducer-microphone mounting apparatus.

A: Ultrasonic transducer

B: Transducer mount

C: Tapered horn

D: Bonding tool

E: Capacitor microphone

F: Tapered tip

G: Graduated scale for rotation

$\mathrm{H}$ : Y-axis micrometer

I: $X$-axis micrometer

$\mathrm{J}$ : Z-axis micrometer 
It is to be expected that the vibration characteristics of the tool set to a normal extension will be modified somewhat during bonding because of the loading on the tool; the mechanical $Q$ of the loaded transducer-tool combination will be lowered. Preliminary measurements of the standing wave pattern of long ( 0.828 in.) bonding tools under load show that the position of the lowest node shifts only a small amount ( 0.015 in.). The shift was found to be in a direction away from the end of the tool. The observed shift was sufficiently small that it alone does not appear to affect the maximum vibration amplitude at the tip. Under this load condition, the resonance curve broadens and the effective $Q$ is reduced. Thus for the $0.828^{\prime \prime}$ tool, the ability to maintain the tool tip amplitude constant under load conditions depends to a large degree on the feedback characteristics of the transducer power supply. (See also section 3.1.4-5)

\subsubsection{Transducer-Mount Interaction Problems}

Some transducer-mount problems with respect to bonding machine tune-up procedures were given in section 2.1.4. Knowledge of the existence of such problems led to the following study to characterize and understand them.

The effects of mount design on the vibration amplitude of the bonding tool tip were examined. As was seen in Fig. 2-9, the transducer-tool assembly is held in a fixed mount. It is essential that this be done in such a way that absorption of ultrasonic energy into the mount is minimized. A transducer manufacturer has provided tables [7] of design dimensions which should be avoided for various materials because of the possibility of resonant reflections.

Different transducer mounts were made and tested with several commercial transducer-tool assemblies. In one poor mounting condition, the wave form shown in Fig. 2-11 was observed. This wave form apparently results when some of the transducer energy is absorbed into the mount at a subharmonic rate. Under another condition, hash was observed at the peak of an otherwise normal sine wave. An improperly mounted transducer can have different resonance frequencies as the applied power is changed. Al so energy can be absorbed by screws on the mount, near but not necessarily contacting the transducer. Since polytetrafluoroethylene (PTFE) is an excellent ultrasonic damping material, this absorption can be reduced by wrapping each screw with PTFE plumbing pipe-tape before insertion.

These problems do not depend solely on the mount characteristics, but rather on the transducer-mount combination. In a particular transducer mount that was carefully designed to avoid resonant dimensions, one commercial transducer lost energy by subharmonic absorption, but another transducer from a different commercial source functioned satisfactorily. It was also noted that most of the above characteristics are power 

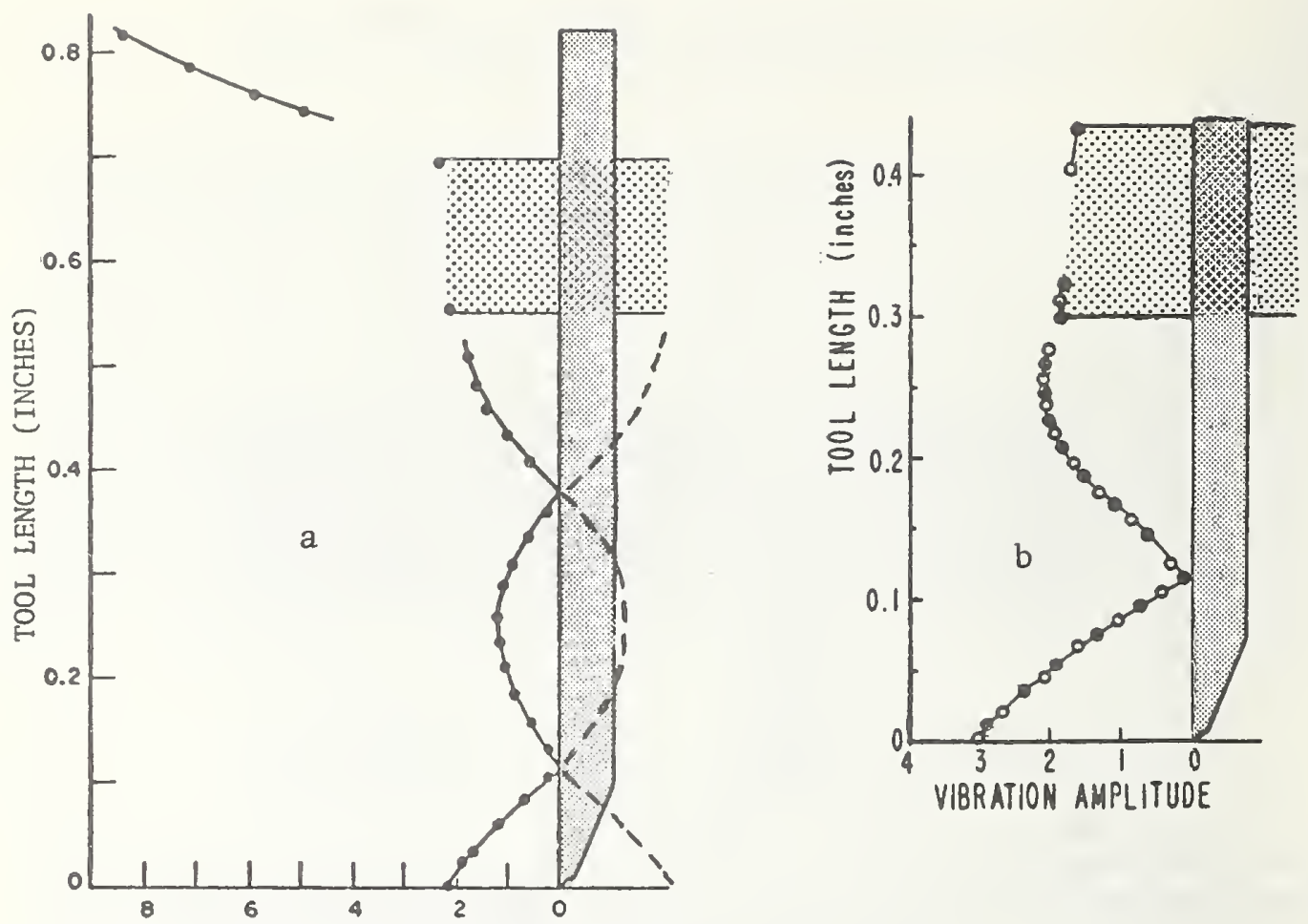

VIBRATION AMPLITUDE (RELATIVE VALUES)

Fig. 2-10. The unloaded vibration modes of two normal tungsten-carbide ultrasonic-bonding-tools set at typical extensions below the transducer horn. The dotted area near the top of each figure represents the physical position of the transducer, and its extension to the left of the bonding tool indicates its relative vibration amplitude. The symmetry of the vibration is indicated by the dashed curve in Fig. 2-10a. This mirror image curve is omitted in most subsequent figures.

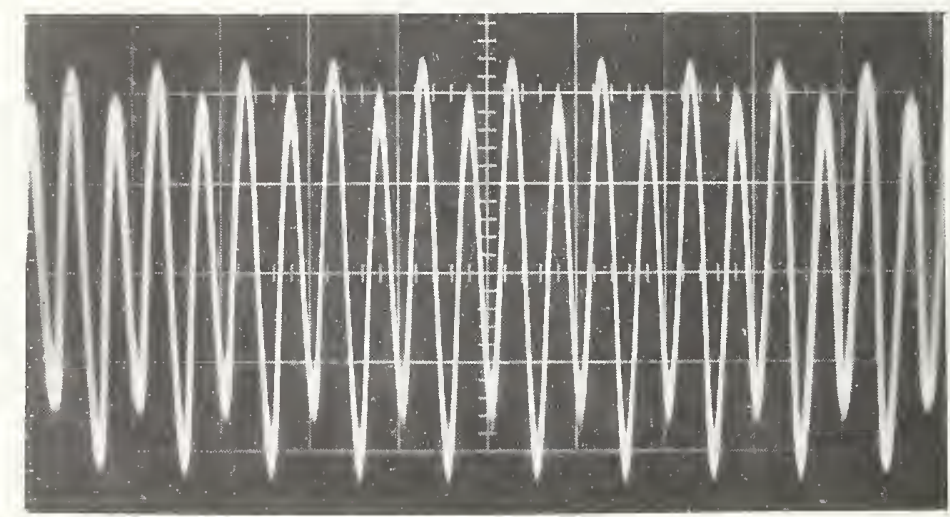

Fig. 2-11. Unusual wave form obtained with the capacitor microphone. This wave form results from a transducer mount resonance that absorbs power at half the excitation rate ( $30 \mathrm{kHz}$ rate when the transducer is driven at $60 \mathrm{kHz}$ ). Similar patterns have resulted from loose bonding tools. 
sensitive. Below some threshold there is usually no evidence of absorption into the mount. As the power control setting is increased, more power may be absorbed into the mount instead of driving the tool. At still higher levels this power absorption may decrease. As a result, it cannot always be assumed that the vibration amplitude of the tool tip will always increase at the same rate as the power control setting.

It has also been verified that these effects can exist on commercial ultrasonic bonding machines. Direct observation of the vibration amplitude of the tool tip by means of a capacitor microphone has been shown to be an effective means of establishing whether or not power is being transmitted to the tool.

(G. G. Harman)

\subsubsection{Modification of the Transducer $Q$}

Since mechanical resonance curves measured with a capacitor microphone have been very useful in characterizing ultrasonic wire bonders, a simple system of generating them was constructed. A small, variable capacitor with a linear potentiometer attached to its shaft is connected across the oscillator coil of the ultrasonic power supply. The potentiometer is used to control the voltage which is applied to the horizontal input of an oscilloscope. When the capacitor is manually tuned, a complete resonance curve is generated, picked up by the microphone, and displayed on the oscilloscope. Such a system permits the rapid comparison of resonant curves taken under different conditions.

In order to lower its mechanical Q, a series of holes was drilled in the horn of an older transducer as shown in Fig. 2-12a. The resonance curve with the unmodified transducer obtained with the above system is shown in Fig. 2-12b. The initial Q of this particular transducer-tool-power supply combination was 165. After the modification, the Q dropped to 125 as shown in Fig. 2-12c, a decrease of approximately 25 percent. The mechanical resonant frequency decreased $2.6 \mathrm{kHz}$. For both measurements the same bonding tool and power supply were used and the bonding tool extension and power supply settings were reproduced as accurately as possible. Thus it can be seen that by means of a simple modification the mechanical $Q$ of an existing transducer can be lowered significantly while the frequency and amplitude remain essentially unchanged. The broader resonance peak obtained in this way reduces the detuning effects of thermal environmental changes and instabilities in the oscillator.

(H. K. Kessler) 


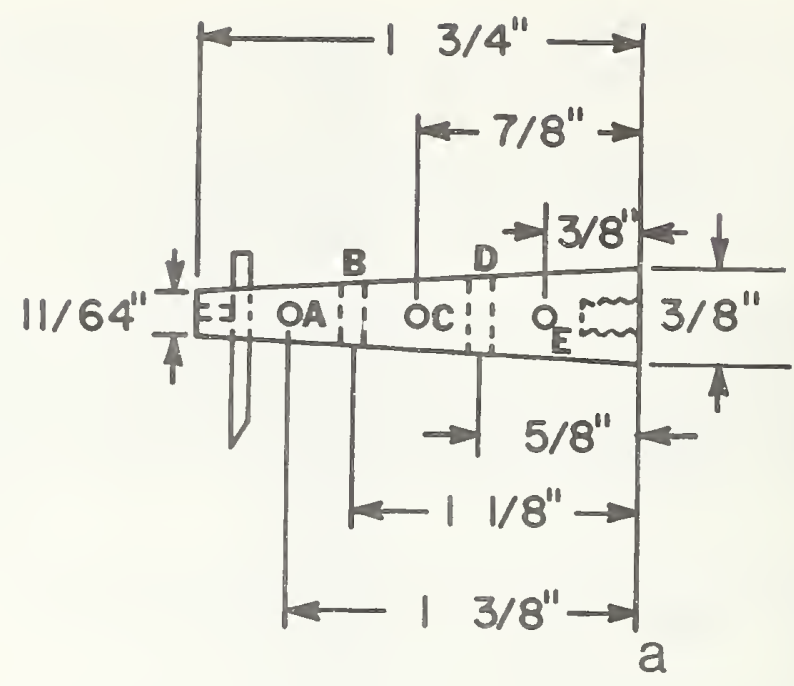

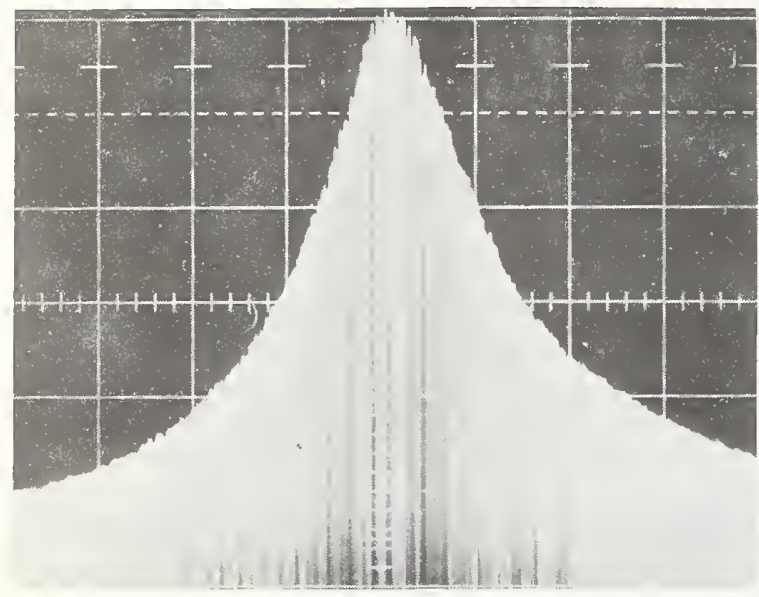

b

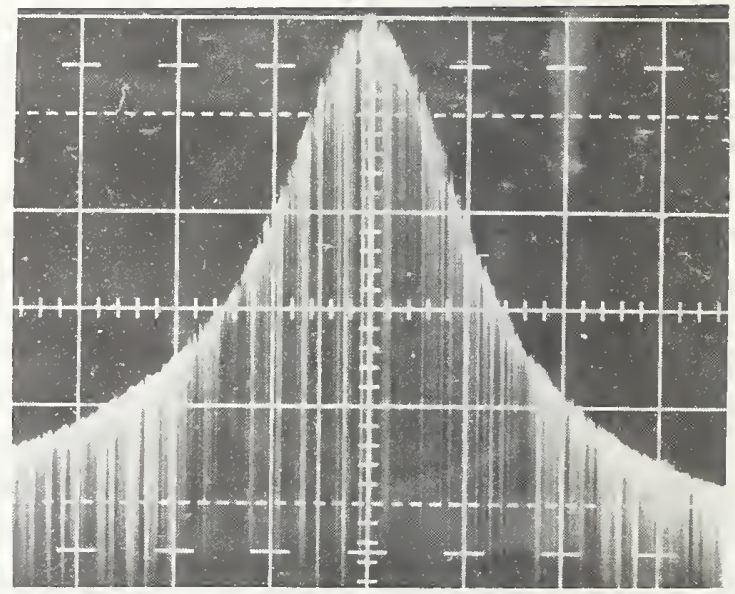

C

Fig. 2-12. Modification of transducer horn to reduce the mechanical $Q$. a: Sketch of hole placement. (A, B, C, and D: 0.080-in. diameter holes, E: 0.120-in. diameter hole.)

$\mathrm{b}$ : Resonance curve of unmodified transducer. (Horizontal scale - $250 \mathrm{~Hz} / \mathrm{div}$.

c: Resonance curve of modified transducer. (Horizontal scale - $250 \mathrm{~Hz} /$ div.) 


\subsubsection{Resonance Dominated by a Critical \\ Extension of the Tool}

A dramatic indication of the effect of tool extension can be seen from the frequency dependence of the tip vibration amplitude as shown in Fig. 2-13 and 2-14. For the $0.375-$ in. $(9.5-\mathrm{mm})$, tool extension there is a double resonance peak with very large amplitudes at the resonances. After removal and reinsertion of the tool to an extension of 0.325 in. $(8.2 \mathrm{~mm})$, the curve with a single peak which has amplitude, resonant frequency, and shape typical of normal bonding is observed. The standing wave pattern observed with the bonder tuned to the $59 \mathrm{kHz}$ resonance peak of Fig. 2-13 is shown in Fig. 2-14. In this case the vibration amplitude at the tool tip is the largest amplitude measured during this series of experiments, but the vibration amplitude of the upper portion of the tool is smaller than usually observed. The power control setting was that normally used for bonding $0.001-i n$. $(25-\mu \mathrm{m})$ diameter aluminum wire.

The results of these studies confirm that the normally stated unloaded electrical $Q$ of a transducer has no significance insofar as the vibration characteristics of the bonding tool are concerned. Not only does the mechanical $Q$ of a particular transducer-tool combination depend on the method of electrical drive (section 2.3.10) but it also depends on the length and extension of the bonding tool. The mechanical $Q$ may be changed as much as a factor of 2 by varying the tool extension within the range specified by the manufacturer.

\subsubsection{Bonding Tool Vibration Amplitude Variations Resulting from Removal and Replacement of the Tool}

The amplitude of ultrasonic vibration along the length of the bonding tool was studied on many different tools inserted to various extensions. The standing wave patterns in Fig. 2-15 demonstrate the changes which may occur when the same tool is removed from the horn and reinserted to a slightly shorter tip extension. The tool tip vibration amplitude changes substantially as does the amplitude of the standing wave pattern above and below the horn. The same power setting was used for both measurements, and the driving frequency required to maximize the tip vibration amplitude changed only slightly (about $50 \mathrm{~Hz}$ ).

As shown in Fig. 2-16 similar changes occur when the tool is removed and reinserted to within 0.002 in. of the same extension. The results of a similar experiment carried out on a short tool driven by a different transducer and type of power supply are shown in Fig. 2-17. In this case the tip amplitude remains essentially the same; however, the standing wave pattern changes markedly. 


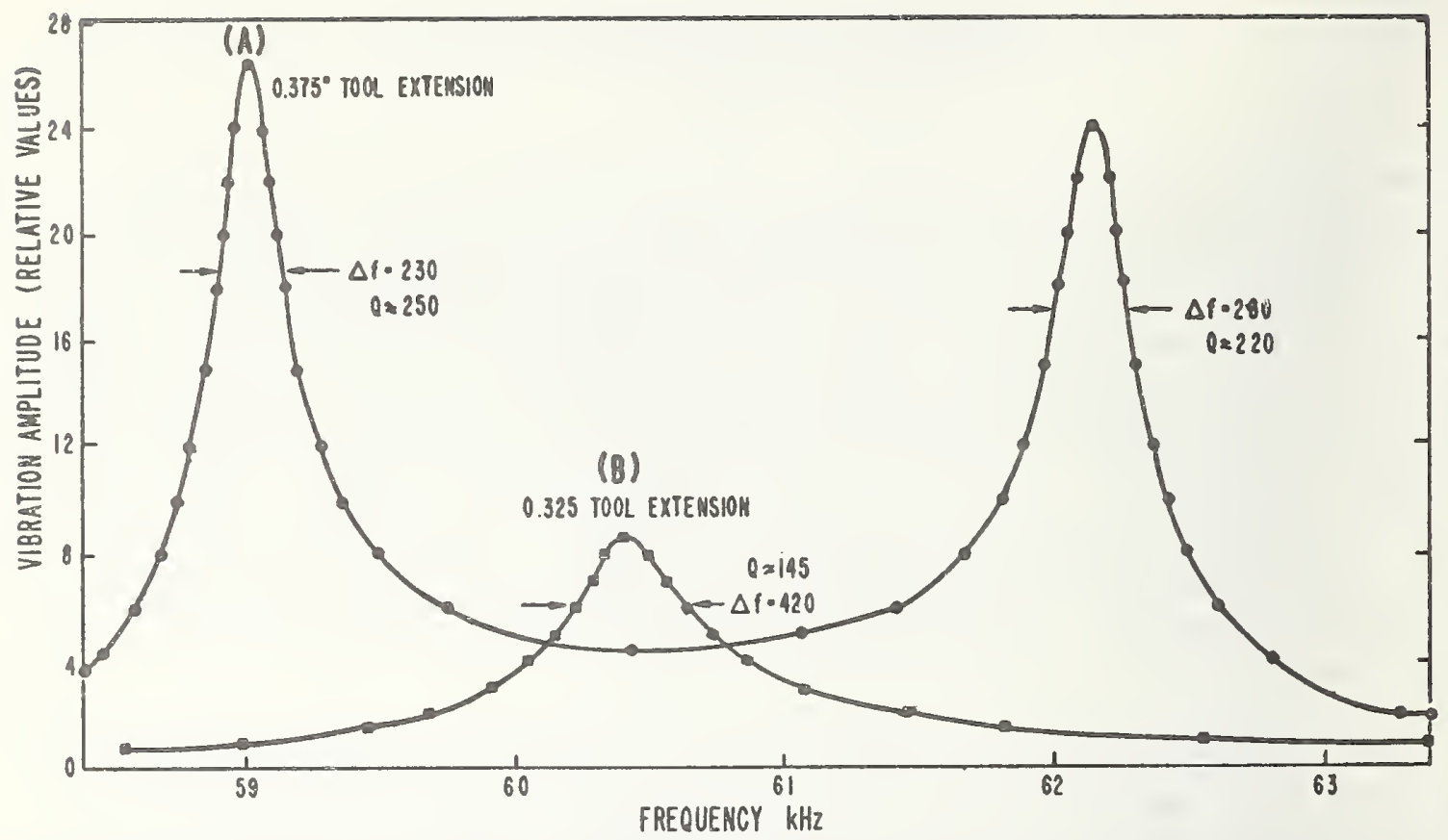

Fig. 2-13. Mechanical resonance curves for the same tool set at two different extensions below the horn. The control setting of the ultrasonic power supply was identical for both curves. The maximum amplitude in curve $B$ is typical of conditions used for bonding 0.001-in. aluminum wire.

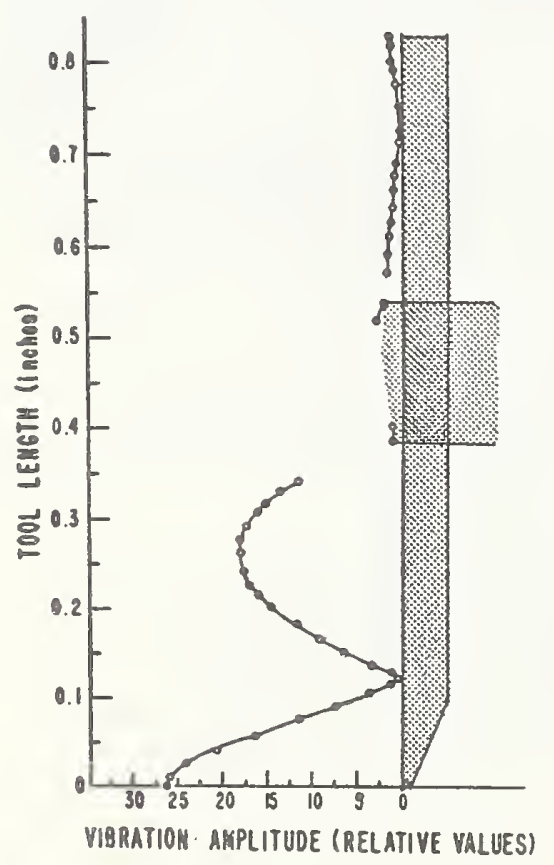

Fig. 2-14. Vibration modes of a long ultrasonic bonding tool which demonstrates unusually large resonance effects. These measurements were made with the ultrasonic system tuned to the $59 \mathrm{kHz}$ resonance peak of Fig. 2-13 curve A. Amplitudes are relative values consistent within a given figure; they cannot be compared directly with other figures. The amplitude and position of the transducer horn (the dotted portion) are measured at its tip. Since the horn is tapered, the tool extension cannot be precisely measured from the figure. 


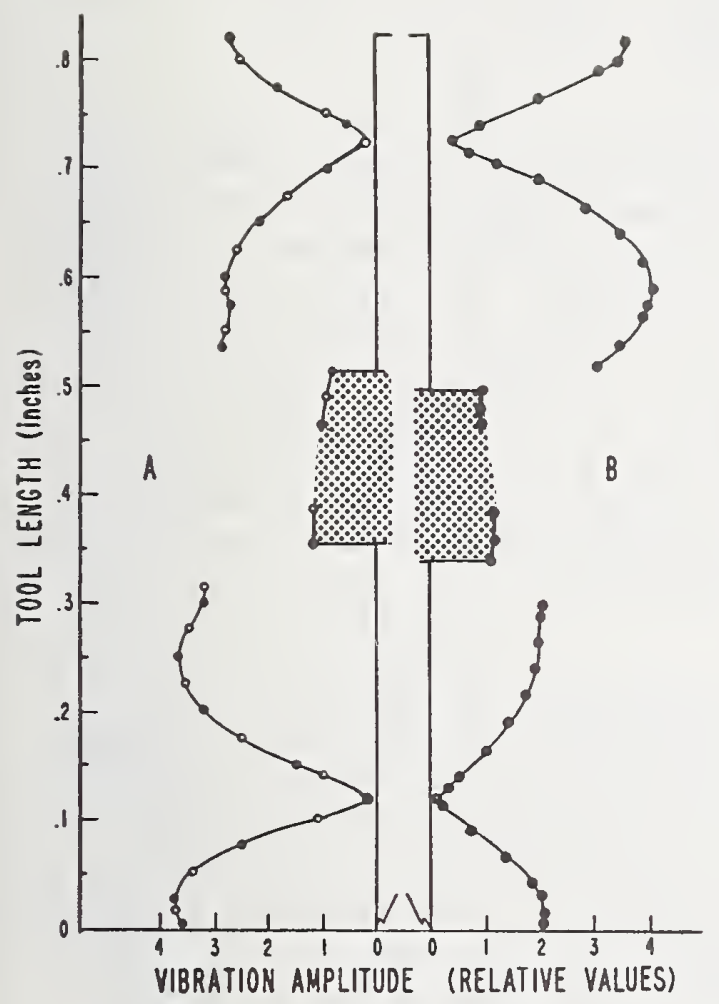

Fig. 2-15. Vibrational amplitude of a long ultrasonic bonding tool.

A : A bonding tool extended 0.350

in. below the horn.

B: The same bonding tool extended $0.335 \mathrm{in}$. below the horn.

The vibrational amplitude of the horn, which is in general less than that of the tool, is shown in the center part of the figure. Data for both extensions were taken with the same power setting on the ultrasonic supply, although the frequency was retuned slightly for maximum amplitude in each case. Amplitudes are relative values consistent within a given figure; they cannot be compared directly with other figures. The amplitudes and position of the horn are measured at its tip. Since the horn is tapered, the tool extension cannot be measured from the figure. Rounding of the amplitude curve at the tool extremities can be attributed to edge effects.

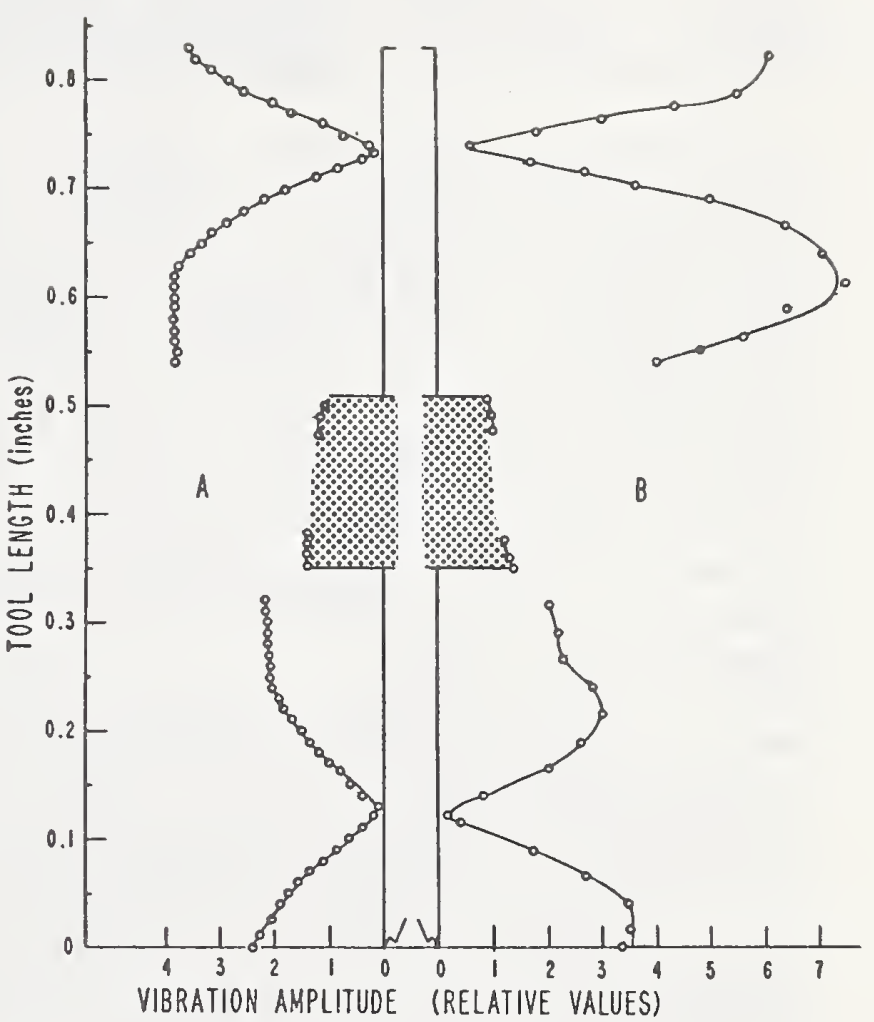

Fig. 2-16. Vibrational amplitude of a long ultrasonic bonding tool. The tool was removed after taking the data for $A$ and then replaced to the same extension for taking the data for $B$. The vibrational amplitude of the horn, which is in general less than that of the tool, is shown in the center part of the figure. Data for both extensions were taken with the same power setting on the ultrasonic supply, although the frequency was retuned slightly for maximum amplitude in each case. Amplitudes are relative values consistent within a given figure; they cannot be compared directly with other figures. The amplitudes and position of the horn are measured at its tip. Since the horn is tapered, the tool extension cannot be measured from the figure. Rounding of the amplitude at the tool extremities can be attributed to edge effects. 
It appears that once a tool is firmly clamped in position, it will maintain the same tip motion for given drive and temperature conditions, until removed. This suggests that it would be feasible to establish the bonding schedule from measurements of tip vibration amplitude after each tool change or adjustment. Either a capacitor microphone or a magnetic pickup could be used for this purpose (section 1.1.1 and 1.1.2).

A series of experiments was conducted to establish the origin of the variability in the vibration amplitude of the tool tip and to ensure that the variability was not due to faulty measurement equipment or techniques. Measurements on tools which have not been moved can be repeated to within about 5 percent. It is apparent that the principal difficulty rests with irregularities in the shape of the tool hole in the transducer horn, and with irregularities in the set screw which clamps the tool in place. A typical hole in a relatively new transducer horn is shown in Fig. 2-18. Irregular ridges with a height about 0.001 in. $(25 \mu \mathrm{m})$ can be readily seen, and there is evidence of metal bent outward during the threading of the set screw hole. Because of these irregularities, the tool would not necessarily be driven from the same point on its length each time it was reinserted and clamped. This is equivalent to inserting the tool to a different depth each time even though the gage block measurement between horn and tool tip remains unchanged. Furthermore, the set screw wears with use and probably will not always clamp the tool in the same position. Some improvement can be obtained if the tool holes were drilled, reamed, and polished. Even under such conditions, a tolerance of $\sim 0.0002$ in. ( $5 \mathrm{\mu m}$ ) is about the best that could be expected, and this, combined with a similar tolerance which is customarily specified on the radius of tungsten carbide bonding tools can result in a misfit many times the vibration amplitude. After prolonged use the tool may wear the edge of the hole and increase the problem.

There are several possible solutions to these problems. A simple remedy for set screw wear is to replace it every time the bonding tool is changed. It was also found that a more reproducible contact between the set screw and tool can be achieved if the end of the set screw is filled with a soft material such as lead or copper. Such a screw, or at least the filling, would still have to be replaced periodically. Various methods of rigidly holding the tools, such as epoxying or brazing, appear to be impractical. The misfit problem might be reduced by filling the empty regions with a glue-like material that transmits $60 \mathrm{kHz}$ vibration and can be put in and removed easily. It is also possible that a replaceable metal-foil gasket could be used for this purpose. The geometry of the hole could be modified by enlarging it everywhere except directly behind the set screw so that all motion originates from a much smaller area.

(G. G. Harman) 


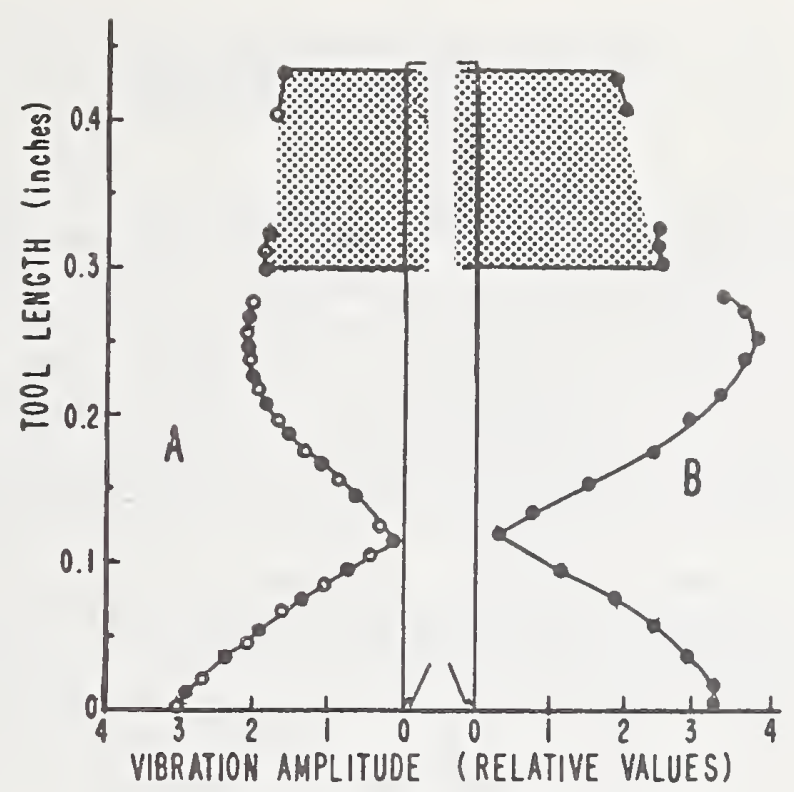

Fig. 2-17. Vibration modes of a short ultrasonic bonding tool. The tool was removed after taking the data $A$ and then replaced to the same extension for taking the data B. The vibrational amplitude of the horn which is in general less than that of the tool tip, is indicated by the dotted part of the figure. Data for both extensions were taken with the same power setting on the ultrasonic supply, although the frequency was retuned slightly for maximum amplitude in each case. Amplitudes are relative values consistent within a given figure; they cannot be compared directly with other figures.

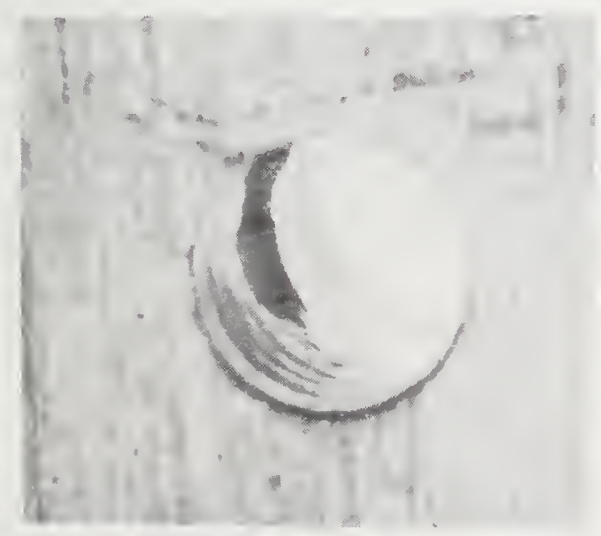

Fig. 2-18. Hole in an ultrasonic transducer horn through which the bonding tool is inserted. The dark area on the left is a part of the thread for the set screw. The photograph clearly shows irregularities on the wall of the hole. Magnification is approximately 18 times. 
[527] Previous1y published work has shown that slight changes in the power supply impedance can have a significant effect on the mechanical $Q$ of the wire bond system (NBS Tech. Note 495, pp. 26-28). These measurements were made with special laboratory amplifiers in which the current or voltage was individually controlled. Similar measurements have now been made with three commercially available ultrasonic wire bonding power supplies which were used to drive the same transducer-tool combination. The vibration amplitude was measured with a capacitor microphone* positioned near the tip of the bonding tool. Mechanical resonance curves obtained with these three power supplies are shown in Fig. 2-19. As would be expected from the earlier results, it was found that the mechanical $Q$ of the system was lowest when driven with a constant current source (curve a), intermediate when driven with a constant power source (curve c), and highest when driven with a constant voltage source (curve b). For these tests, a 0.828-in. (21-mm) long bonding tool was used with an extension of 0.555 in. (14 mm) below the horn, which is commonly used on some bonders.

[495] In addition to the main resonance curves around the normal $60-\mathrm{kHz}$ operating frequency, it was found that there were from 13 to 18 other resonances between 7 and $90 \mathrm{kHz}$. Different bonding tool extensions slightly affect the main $60-\mathrm{kHz}$ resonant frequency but the existence of many other resonances are strongly dependent on specific tool extension lengths.

(G. G. Harman)

\subsubsection{The Swept Frequency Mode of Power Supply Operation}

[495] The capacitor microphone was used to examine the bonding tool tip vibration amplitude while the transducer was driven in the swept-frequency mode with a medium $Q$ system. The envelope of the displacement amplitude for three values of the center frequency, $f_{0}$, is displayed in Fig. 2-20. The shape of the envelope can be changed significantly by relatively small changes in the center frequency. Thus, to maintain reproducible bonding conditions, it is necessary both to have a stable oscillator and to exercise care in tuning the ultrasonic system, even when the sweptfrequency mode is used. Frequency modulation of the power supply oscillator is often used to reduce the problems associated with thermal drift and oscillator instabilities, but as seen in Fig. 2-20 more problems may be created than solved.

* It is essential that the microphone have a flat frequency response in the 59-63 kHz range [see NBS Tech. Note $573 \mathrm{p} .5$ ], otherwise the resonance curves will appear skewed and/or flat on top. 


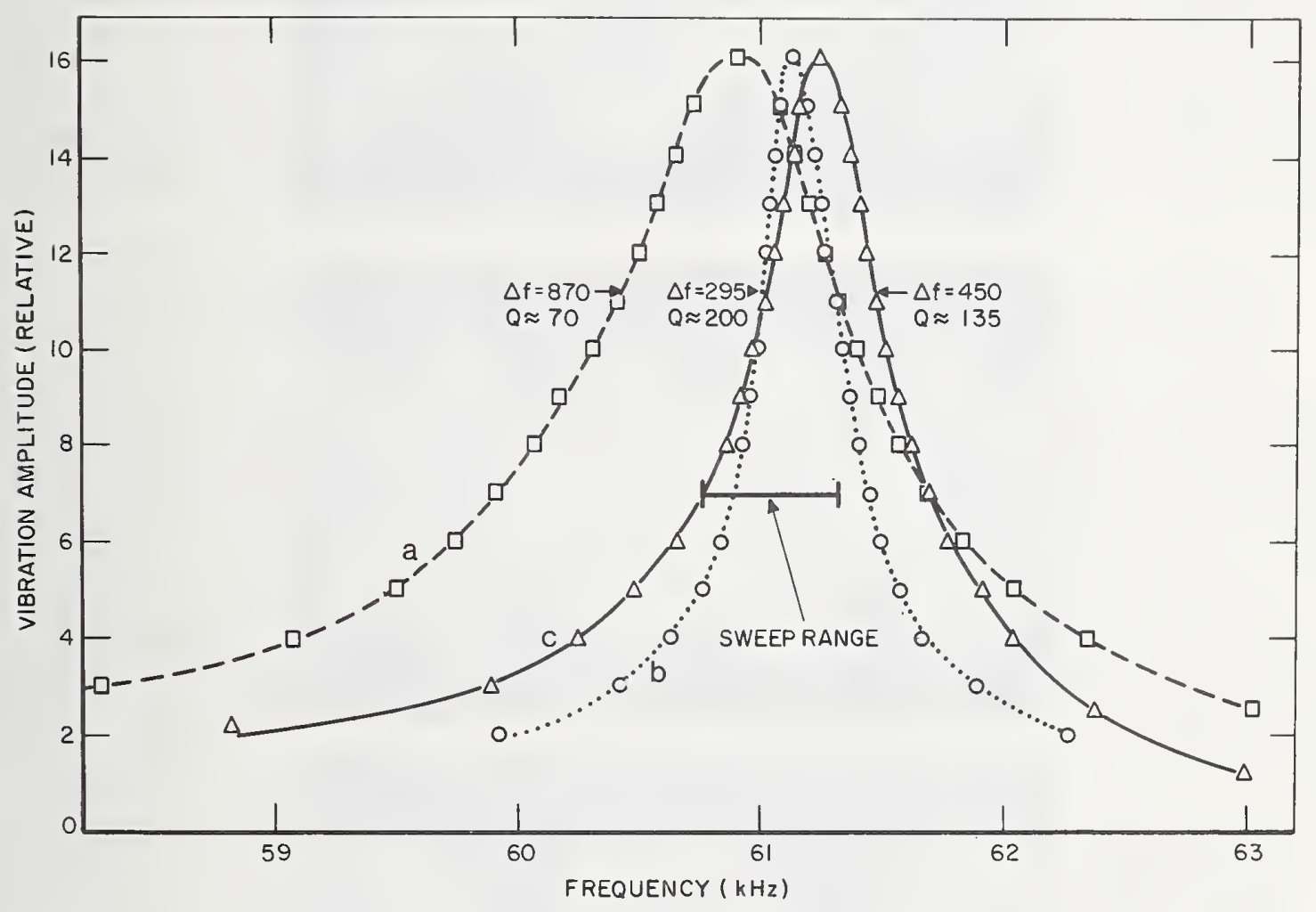

Fig. 2-19. Mechanical resonance curves taken with a capacitor microphone using the same tool and transducer, driven by three different ultrasonic power supplies. In each case the tool extension is 0.555 in.

a: Constant current source.

$\mathrm{b}$ : Constant voltage source.

c: Constant power source. (The sweep range indicated is that provided with this supply, see Fig. 2-20 and -21d.) 

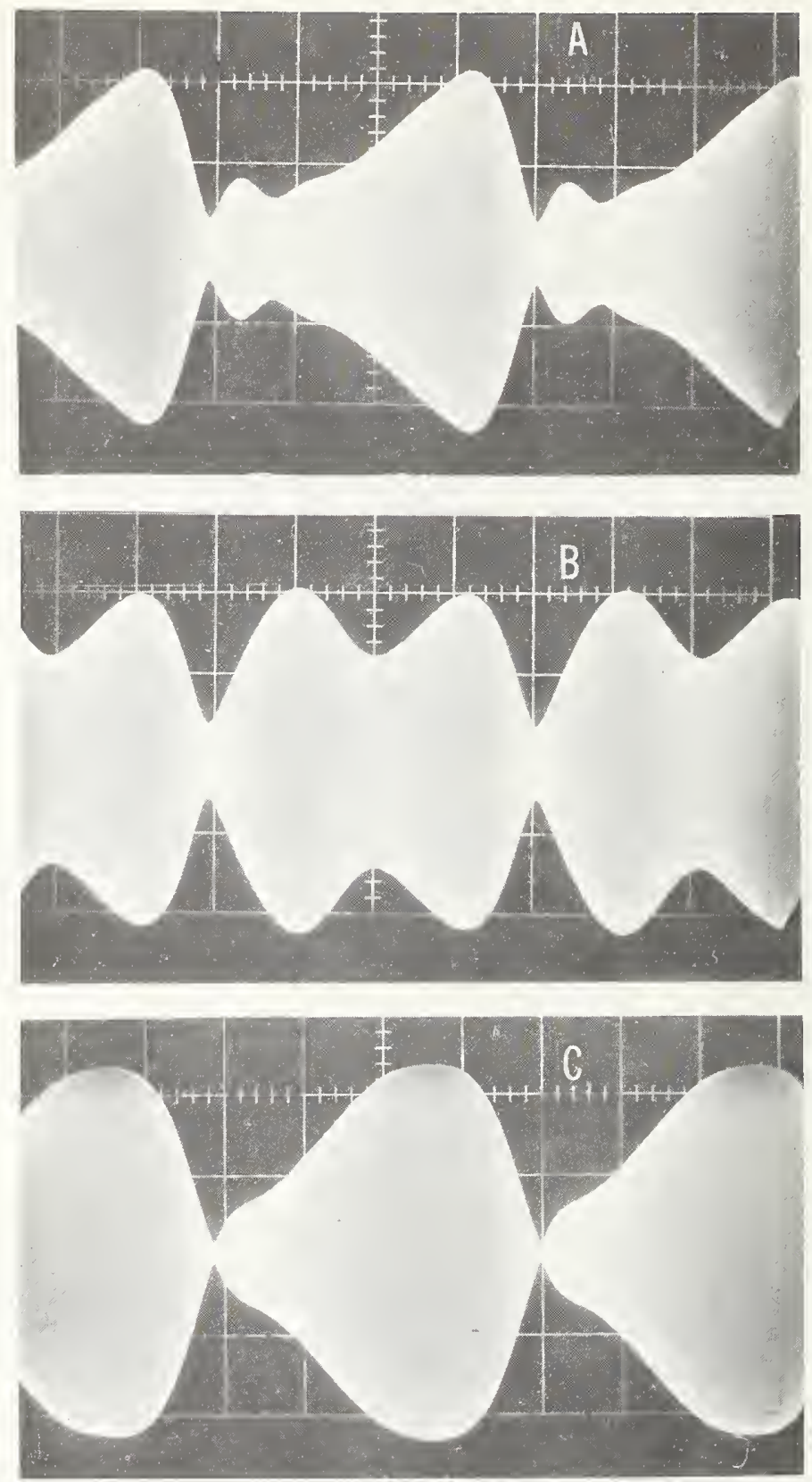

Fig. 2-20. Three oscillograms of the sound pressure from the tip of a bonding tool. These patterns result from sweeping the frequency of the power supply by approximately $1200 \mathrm{~Hz}$. The vertical axis is proportional to bonding tool displacement. The horizontal axis is time ( $1 \mathrm{~ms} /$ division). The sweep period is about $4 \mathrm{~ms}$.

A: $\quad f_{0}=60.50 \mathrm{kHz}$ (manufacturer's specification).

B: $f_{0}=61.00 \mathrm{kHz}$ (system was tuned to give maximum ultrasonic output without sweeping).

$c: \quad f_{0}=61.38 \mathrm{kHz}$ (pattern least affected by slight detuning of the center frequency). 
In order to understand the various effects displayed in Fig. 2-20, particularly the waveshape asymetries, various frequency and amplitude modulations were applied to the power supply oscillator and the results were studied by monitoring the bonding tool tip vibration.

The vibration amplitude response to a square-wave modulation voltage which turns the $60 \mathrm{kHz}$ supply on and off sharply is shown in Fig. 2-21a. The rise time ( 10 to 90 percent) was $1 \mathrm{~ms}$ whereas the rise time of the modulating pulse was $<<0.1$ of this value. The mechanical Q calculated from the time required for the amplitude to decay to one half its equilibrium value agrees very well with the measured static $Q$ of the same system (curve c, Fig. 2-19), approximately 135.

A linear frequency drive was introduced into the power supply oscillator by means of a triangular-wave modulation voltage with the result shown in Fig. 2-21b. The frequency changed linearly with voltage, but the vibration amplitude envelopes for increasing and decreasing frequency were not the same. The decreasing frequency envelope resembles a normal resonance curve; however, the increasing frequency envelope was compressed toward the high frequency end. This effect is presumed to result from the slow mechanical rise time of the system. In the increasing frequency case, not only the tool tip amplitude but also the frequency is increasing. Thus the net acceleration of the motion is significantly higher, equivalent to frequency modulation at a considerably higher sweep rate. Conversely, by sweeping toward lower frequencies, a lower mechanical acceleration is applied to the system, and a more nearly quasi-static sweep results.

If the slow mechanical response of the system is taken into account, it is possible, by adjusting the sweep rate, amplitude, and center frequency, and by using a saw-tooth modulation voltage to obtain a symmetrical system response curve that is closely related to the static resonance curve, c, of Fig. 2-19. The result shown in Fig. 2-21c was obtained on the same transducer-tool system with the same tool extension and the same power supply as were used to obtain the static resonance curve.

Studies of the frequency modulation in some commercial power supplies have revealed that the frequency may not always be swept through the entire static resonance peak. In a typical case the sweep starts from the lower frequency side, barely goes to the resonance peak, and then returns toward lower frequencies. The result shown in Fig. 2-21d was obtained when the transducer system was tuned by the manufacturer's recommended method. The sweep range for this case was shown in Fig. 2-19c. Such a partial sweep may create problems. If the ambient temperature changes, causing the resonant frequency of the oscillator or the transducer to drift, then the sweep frequency may not reach the top of the mechanical resonance curve and the vibration amplitude will be too low for bonding.

(G. G. Harman) 


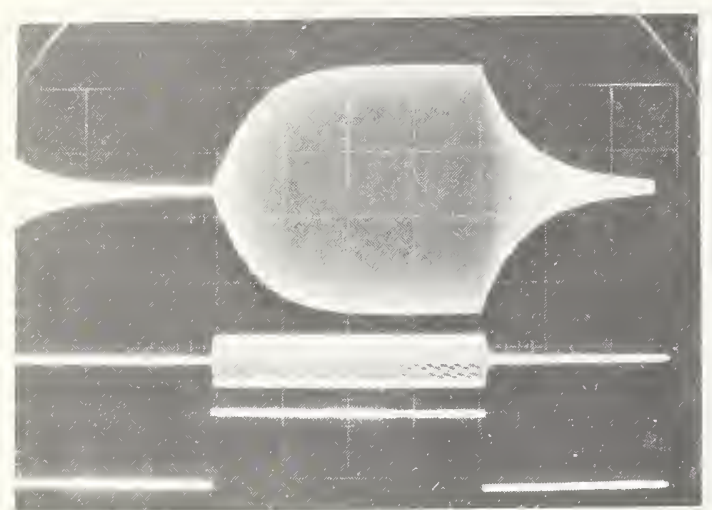

a: Square-wave modulation.
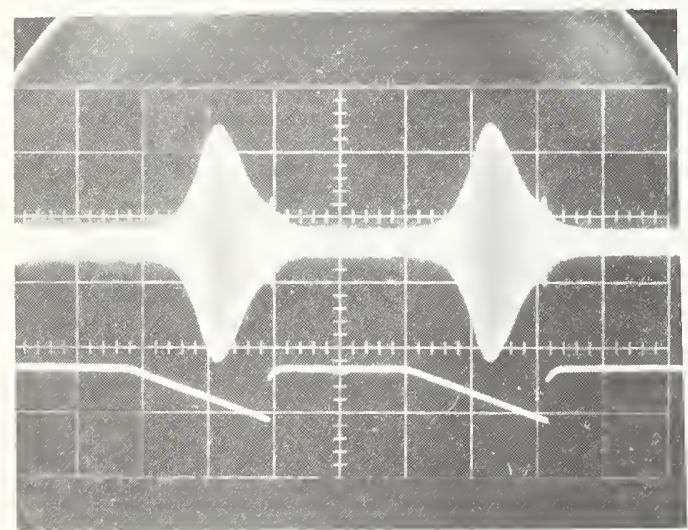

c: Saw-tooth modulation (decreasing frequency).

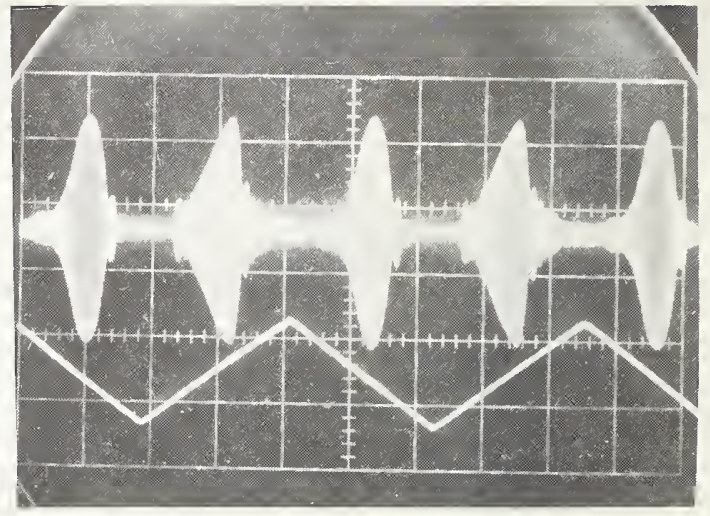

b: Triangular-wave modulation.

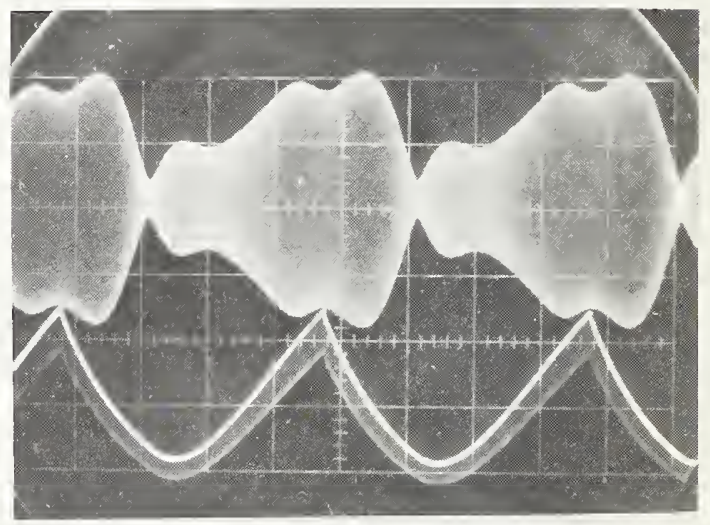

d: Modulation wave-form supplied with power supply.

Top curve: Vibration amplitude of the tool tip as measured with a capacitor microphone.

(Vertical scale $-0.5 \mathrm{mV} / \mathrm{div}$ )

Bottom curve: Modulation voltage applied to oscillator.

(Vertical scale - a: $0.5 \mathrm{~V} / \mathrm{div}$

b, c: $1 \mathrm{~V} / \mathrm{div}$

$\mathrm{d}: 0.1 \mathrm{~V} / \mathrm{div})$

Center curve (a only): Power supply oscillator output voltage. (Vertical scale $-2 \mathrm{~V} / \mathrm{div}$ )

(Horizontal scale - a, d: $1 \mathrm{~ms} / \mathrm{div}$;
b, c: $5 \mathrm{~ms} / \mathrm{div})$

Fig. 2-21. Response of the bonding tool vibration amplitude to frequency modulation of the power supply oscillator 


\subsubsection{Studies of a Commercial Phase-Locked-Loop}

An investigation was made of a five-watt ultrasonic power supply-transducer system that incorporated a self-tuning phase-locked-loop circuit to keep the system peak-tuned during bonding. The manufacturer no longer produces ultrasonic equipment but many of these units are in the field, so this study will serve to point out possible problems to users and to future designers of such equipment. The system had many problems.

Initial coarse tuning was done by selecting a switch position that gave a maximum indication on the panel meter. The unit could apparently be properly tuned, as indicated by the panel meter, in three of the eight switch positions without a load on the transducer (free vibration). However, it was observed, with a capacitor microphone placed near the tool tip, that only one of these switch positions would result in normal power being delivered to the tool under actual bonding conditions. This situation was more frequently observed when using a bonding machine that required a long (0.828 in., $21 \mathrm{~mm})$ bonding tool rather than the short $(0.425$ in., $10.8 \mathrm{~mm})$ tool and when using a relatively long bonding time (approx. $100 \mathrm{~ms}$ ). The problem is clearly shown in Fig. 2-22, which displays three oscillograms of the $60 \mathrm{kHz}$ bonding-tool-tip vibration envelope. Each oscillogram was made during attempted bonding in which the coarse tuning switch was in one of the three successive positions that produced apparently satisfactory meter tuning under no-load conditions. The first attempted bond (2-22a) was nonadherent. The second bond (2-22b) lifted off with very low bond pull strength, because the phase-1ockedloop became unlocked in the middle of the bonding cycle. The final picture $(2-22 c)$ shows the wave-form envelope of a successful bond which was made with the tuning switch in the third position. Another problem occurred when the power dial-setting was changed significantly. The loop became unlocked, and required a different coarse-tuning switch-position to produce the desirable bonding waveform of Fig. 2-22c.

Obviously, such unpredictable responses as described above can lead to unreliable wire bonds. Conversations with several power supply users indicated that these observations were not unique to the particular power supply that was studied. The reasons for such problems were not determined, but a few general comments are appropriate. An ultrasonic transducer is a complex electro-mechanical device. There is a propagation time delay from transducer to horn to tool and back. This may change the optimum electrical V-I phase relationship during the complexly varying load conditions encountered in bonding. Under those conditions, a particular locked-phase relationship may not lead to the optimum resonant frequency and vibration amplitude.

In principal, at least, the self-tuning phase-locked-1oop approach could offer advantages over the conventional fixed tuned ultrasonic power supplies, particularly for large wire bonding, where the bonding forces are very high. Under such bonding conditions the mechanical resonance of the 
transducer-tool system may change more than a thousand hertz, detuning the system significantly. However, considering the above problems, great care in design and testing of phase-locked-loop power supplies should be carried out before manufacturing them.

(G. G. Harman) 


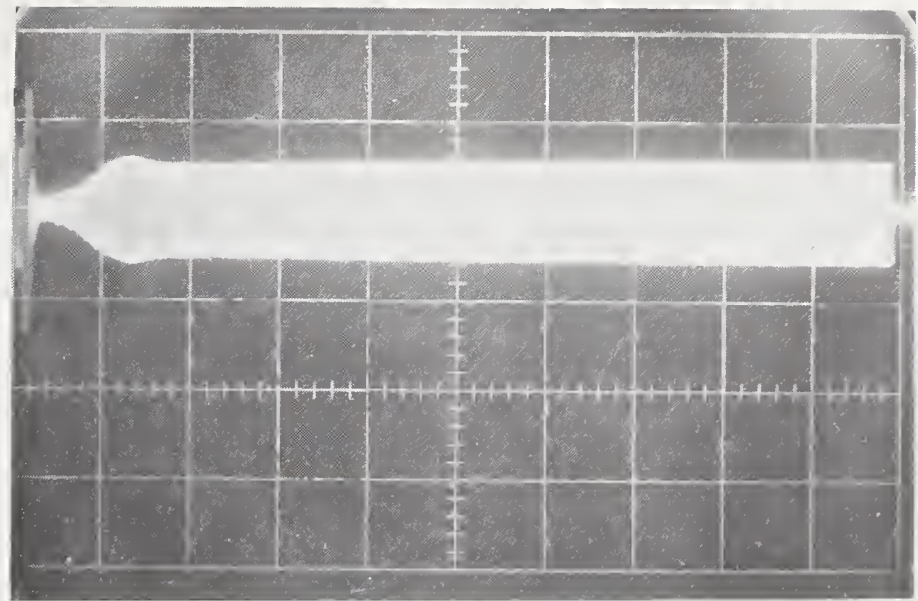

b

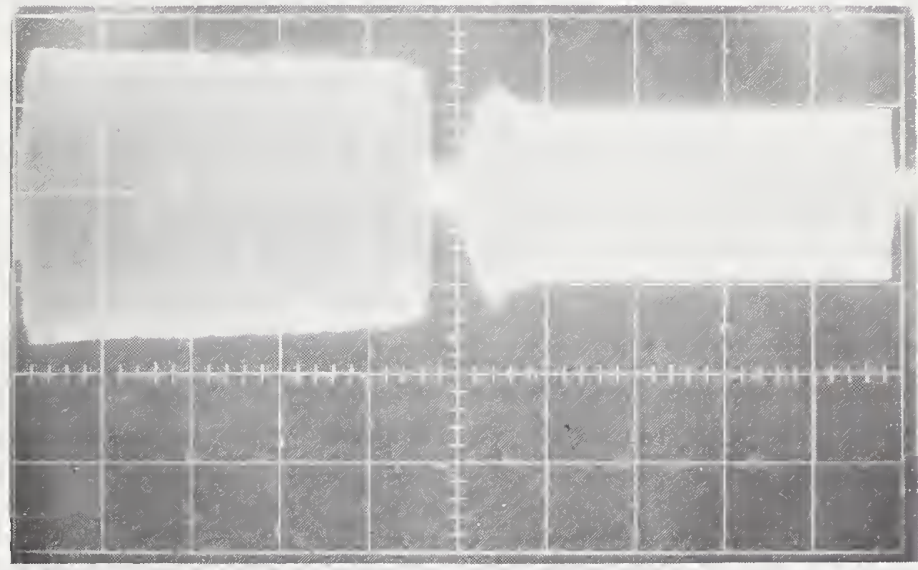

c

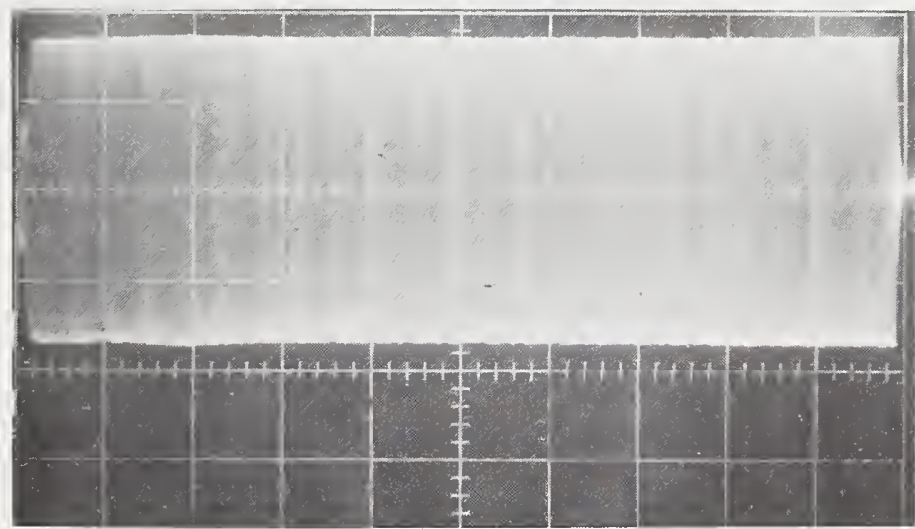

Fig. 2-22. The $60 \mathrm{kHz}$ vibration amplitude envelope detected by a capacitor microphone placed near the tool tip during attempted bonding, using a phase-locked-1oop ultrasonic power supply. Photographs a, b, and $c$ were made with the coarse tuning switch in one of the three successive positions that produced apparently satisfactory meter tuning under no load (free vibration) conditions. Only c produced satisfactory bonds. The vertical scale is $20 \mathrm{mv} / \mathrm{cm}$ and the horizontal scale is $20 \mathrm{~ms} / \mathrm{cm}$. 
There are many variables that can affect the quality of ultrasonic wire bonds. These include the machine parameters of bonding-force, time, and ultrasonic power; the shape and material of the bonding tool, as well as the condition of the wire, the bonding pads; contamination, rigidity of the substrate, etc. Hybrids present special problems, having both thick and thin film bonding pads made up of a variety of metals. Thus it would be desirable to develop measurement methods that can clearly determine when a good metallurgical weld has been achieved, and, possibly using feedback, to alter the bonding schedule automatically and improve a weak bond. In order to achieve this goal it is necessary to understand the mechanism of ultrasonic welding as well as how the various ultrasonic components function during that process. The following section describes various studies pointed toward that goal.

\subsection{In-Process Bond-Quality Studies}

\subsubsection{Preliminary Studies}

[495] Several methods of monitoring ultrasonic parameters during the bonding operation were investigated. The first method used a non-contacting magnetic pickup placed near the tungsten carbide bonding tool. Tools are weakly ferromagnetic since the tungsten carbide particles are cemented with cobalt. The loading of the bonding tool results in decreasing tool velocity or vibrational amplitude and hence an output signal that decreases in amplitude during bond formation. Therefore, this method affords some indication of bond quality. There was some inconvenience in using this system while actually making a bond because the available shielded polarized magnetic pickup coil was larger than desired. Instead of monitoring the tool tip motion it integrates the motion of the entire lower vibration loop of the bonding tool.

Another form of ultrasonic parameter monitoring was studied by observing the $60 \mathrm{kHz}$ voltage applied across the ultrasonic transducer from a relatively high impedance source. The transducer which drives the bonding tool was of the nickel magnetostrictive type having a mechanical $Q$ of about 130. The tool loading during bond formation results in lowered impedance of the transducer and a corresponding change in voltage across the transducer. Thus the information on bond quality obtained by this method is presumably similar to that which would be obtained with the magnetic pickup system.

As a third approach, ceramic transducers were placed at various points on the ultrasonic horn and on the tungsten carbide bonding tool [8]. The bond monitoring information obtained from this system was similar to the other methods. The high-impedance transducer required electrostatic shielding to avoid stray pickup. 
The final parameter monitoring experiment involved the use of a capacitor microphone to detect the ultrasonic sound pressure radiated from the bonding tool during bond formation [9].

The objective of these initial ultrasonic bond monitoring studies was to assemble equipment and survey various possible monitoring methods. No statistical study of the resulting bonds was made and no single method has been chosen as being practical for in-process bond monitoring. All of these methods gave similar qualitative results, although only the microphone gave results in terms of known parameters and offers the possibility of studying the actual tool tip motion. All methods give some information on the bonding tool loading as manifested by a decrease in vibration amplitude due to deformation of the wire. Only a much more intensive investigation can determine the relative sensitivity of each method to subtle changes in bonding conditions.

(H. K. Kessler)

\subsubsection{Beat-Frequency Audio Monitoring}

Experiments were undertaken to investigate the process of making aluminum ultrasonic wire bonds and, if possible, to develop measurement methods that may be applicable to in-process bond quality determination. The initial experiment consisted of mixing a local oscillator with the output of a $1 / 8 \mathrm{in.}(3.2 \mathrm{~mm})$ diameter capacitor microphone having a frequency response greater than $200 \mathrm{kHz}$. The microphone was positioned to pick up sound emitted from the tip of a bonding tool during actual wire bonding. The mixer output was fed into an audio amplifier and from there into a speaker, an oscilloscope, and a tape recorder as shown in the block diagram of Fig. 3-1. This apparatus allows the bonding machine operator to listen to an audible beat frequency sound during bond formation. Typically, with 0.001 in $(25 \mu \mathrm{m})$ diameter aluminum wire, an ultrasonic bond is made in $<100 \mathrm{~ms}$, which is too short a time for the human ear to distinguish small amplitude or frequency changes. Therefore, the audio beat frequency was recorded on magnetic tape and later played back at a slower rate so that details of bonding could be both heard and carefully examined on an oscilloscope. Such recordings were made for the fundamental tool vibration frequency through the fourth harmonic, the extreme response limit of the microphone. A brief investigation extending from the fundamental to the fifteenth harmonic was also carried out using the voltage or current from the ultrasonic power supply driving the transducer. At times the bonding machine schedule was modified by reducing the ultrasonic power and increasing the bonding time so that the bonding process could be heard more clearly in real time.

This initial study was made using two ultrasonic power supplies of different manufacture. However, only one bonding machine, one transducer, and one bonding tool extension were used in the tests. Some variation in the results could be expected if the latter two had also been changed. The initial results were dependent on the particular power supply used to drive the transducer. For power supply A there was a few percent decrease in the $60-\mathrm{kHz}$ tool vibration amplitude during bonding, while for power 


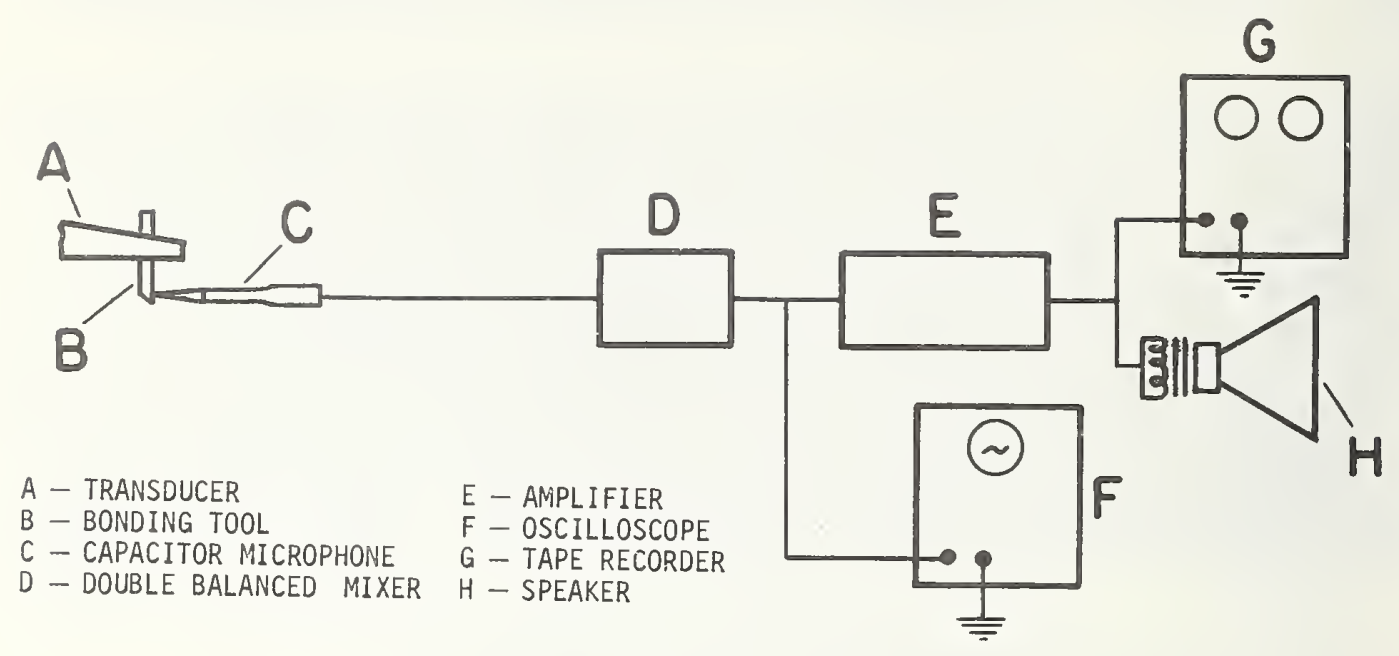

Fig. 3-1. Schematic representation of mixer system for monitoring ultrasonic bond formation.

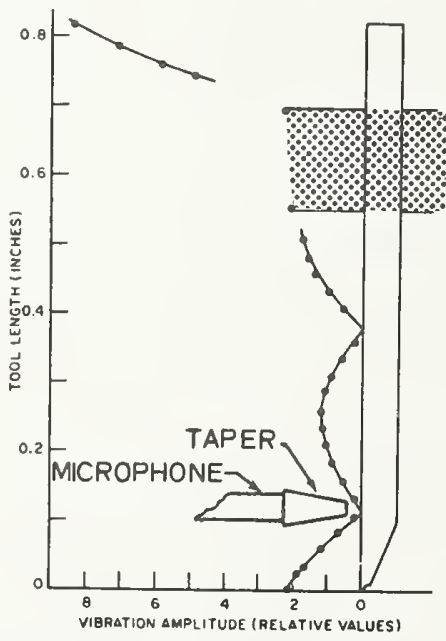

Fig. 3-2. Most desirable placement position for capacitor microphone used in study of variations in vibration amplitudes of the bonding tool at the fundamental driving frequency and its harmonics during bonding. (Measurements may also be made at the upper node, but with somewhat different results.) 
supply $B$ there was a 20 percent increase. These differences are presumably a function of the electronic feedback systems in each power supply as well as the method of transducer drive, which is constant current in one and constant power in the other. As would be expected, differences were observed in the various harmonic relationships during bonding. The second harmonic of both supplies increased in amplitude but in the case of power supply B an unusual frequency warbling was heard during part of each bond. The third harmonic of power supply A was a pure tone that increased 5 to $6 \mathrm{~dB}$ in amplitude as the tool touched the wire or substrate. There was an additional increase of several decibels during actual bonding. These large increases in third harmonic amplitude, which is generated by the changing load on the vibrating tool, are in general agreement with a simple theory of a damped mechanical mass-spring system by Salzer [10].

The results for both power supplies were a function of the microphone pickup position with respect to the vibrating bonding tool. The largest amplitude changes were obtained with the microphone near the bottom of the tool, close to the wire. The smallest changes were observed with the microphone in front of the transducer horn. Similar small changes were also observed when the mixer was connected directly to the electrical drive of the transducer.

Audio monitoring studies of the ultrasonic bonding process were made with a new self-tuning (but not a phase-locked-loop system) transducerpower supply system. During normal bonding, the fundamental frequency was observed to shift by several hundred hertz. This frequency shift was very easily distinguished when the operator listened to the audible beat frequency of the second $(120 \mathrm{kHz})$ or third $(180 \mathrm{kHz})$ harmonic. Qualitative experiments were performed under a number of conditions and on different substrates. For instance when the wire was intentionally placed near the edge of the bonding tool the sound was characteristic of the wire being squeezed out from under the tool when the ultrasonic energy was applied. Different sounds were heard when the bonding took place on a normal aluminum bonding pad, on silicon oxide, or only half on the pad. Many variables were encountered. The most sensitive response was obtained when the local oscillator was on the low side of the particular harmonic chosen (generally the second harmonic was preferred).

(G. G. Harman)

\subsubsection{Harmonic Content and Phased Burst Studies}

A new approach to in process bonding studies that does not involve audio monitoring was initiated. The measurement system consists of a capacitor microphone, with a constricting taper, positioned so that it detects only the $60 \mathrm{kHz}$ sound pressure emitted from the bonding tool in the vicinity of a standing wave node, as shown in Fig. 3-2. This position has the advantage that the $60-\mathrm{kHz}$ fundamental is essentially cancelled out. Since higher harmonics do not share the lower node with the fundamental under these conditions, their relative amplitude is enhanced. For this application, a hole in the microphone taper (Section 1.1.1) of approximately 
0.025 in. $(0.64-\mathrm{mm})$ diameter is most suitable since it straddles the node. Under loaded conditions when the tool is transmitting ultrasonic energy into the bond, the lower node (Fig. 3-2) associated with the fundamental frequency, shifts by approximately $0.015 \mathrm{in} .(0.38 \mathrm{~mm})$. As a result, the amplitude of the fundamental increases by about a factor of two, but the amplitudes of various harmonics increase even more. For this experiment a double-beam oscilloscope was employed so that the entire 50 to $100 \mathrm{~ms}$ bonding period can be displayed on the upper beam, while the waveform in any selected $200 \mu \mathrm{s}$ portion is expanded and displayed simultaneously on the lower-beam. A marker pulse indicating both the position in time and the width of the expanded portion is superimposed on the upperbeam bonding signal. A simplified diagram of the apparatus is shown in Fig. 3-3. A typical oscilloscope pattern, obtained while making a normal bond with $0.001-i n .(25-\mu \mathrm{m})$ diameter aluminum wire, is shown in Fig. 3-4. This bond was made with a constant-amplitude ultrasonic drive applied to the transducer.

Other experiments were carried out in which the amplitude of this drive was briefly increased by a factor of two to four for a short period during the normal bonding time. Fig. 3-5a shows an oscilloscope trace of ultrasonic motion measured at the tip of the vibrating bonding tool rather than at a node. The phased burst of higher amplitude drive is clearly evident. Fig. 3-5b gives the oscilloscope pattern from the node observed while a bond was made with the same bonding schedule as that used for the pattern shown in Fig. 3-4 except that a phased burst was added. Note that waveshape distortion is greatest during the burst period and that the envelope amplitude, a monitor of the nodal shift and deformation, does not increase significantly after the burst. The bond, however, continues to become more adherent as determined by a shear test in which the tool (without ultrasonic excitation) is pushed against a portion of the bond. A study of such bond formation patterns has indicated that the burst resulted in the greatest increase in bond adherence when it was phased 10 to $15 \mathrm{~ms}$ after the initiation of a normal $50 \mathrm{~ms}$ bonding cycle. A typical burst is 2 to $5 \mathrm{~ms}$ long and is empirically adjusted in amplitude so that when it is added to the normal bonding amplitude it does not noticeably increase the bond deformation. The burst duration is adjusted so that the burst alone will not make a bond. These adjustments are not completely independent.

The phased burst appears to increase bond adherence without increased deformation or changed cosmetic appearance. However, this work has been performed as a laboratory experiment and much more work needs to be done to prove that it is useful in a production environment. These experiments were performed with a single ultrasonic power supply-transducer combination because it was easily adaptable to phased-burst operation. Somewhat different oscilloscope displays were obtained when a different make of equipment was used.

(G. G. Harman) 


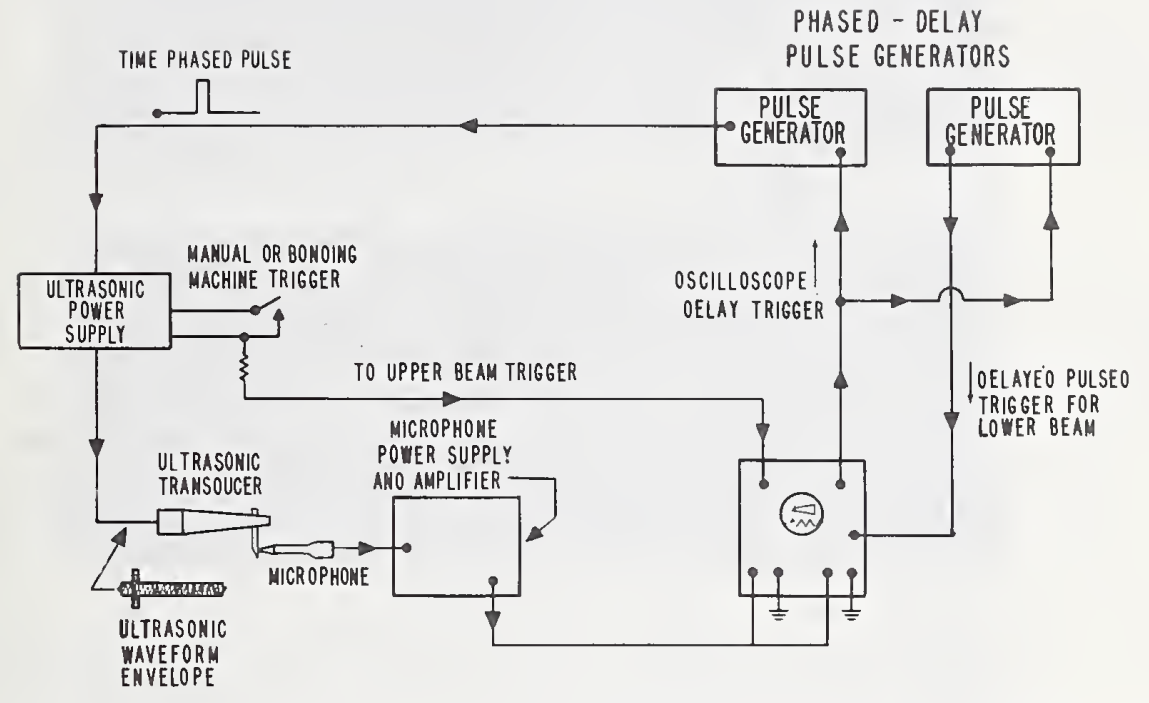

Fig. 3-3. Simplified diagram of the pulse delay and phasing system used to monitor and study ultrasonic bonding.

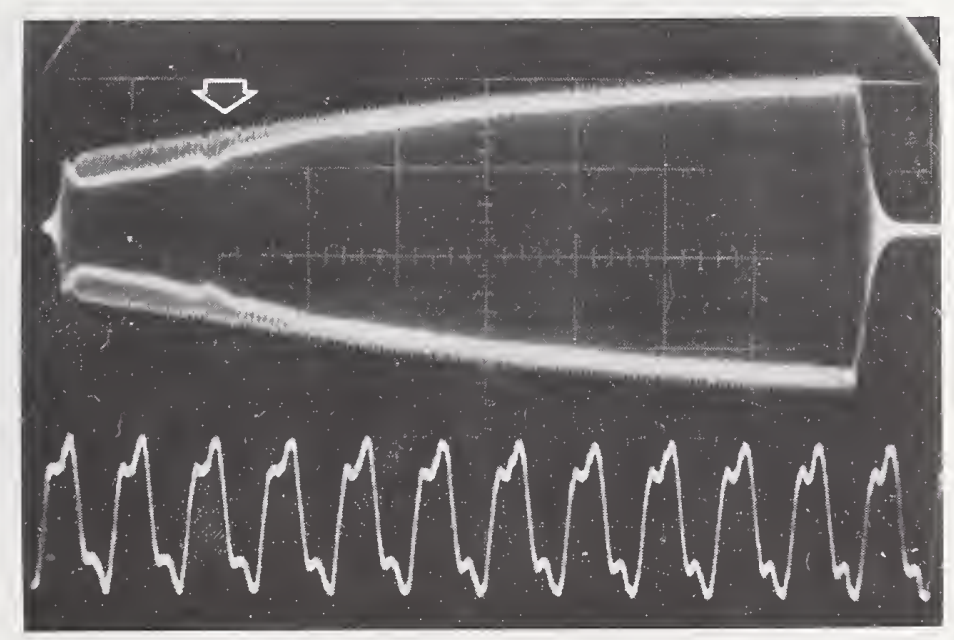

Fig. 3-4. Bond formation pattern of a typical adherent bond.

The upper beam trace shows the entire bonding cycle. Horizontal scale: $5 \mathrm{~ms} / \mathrm{div}$. A portion of this trace indicated by the arrow is expanded on the lower beam trace. Horizontal scale: $20 \mu \mathrm{s} / \mathrm{div}$. Vertical scale for the lower trace is about $4 / 5$ of that for the upper trace. 


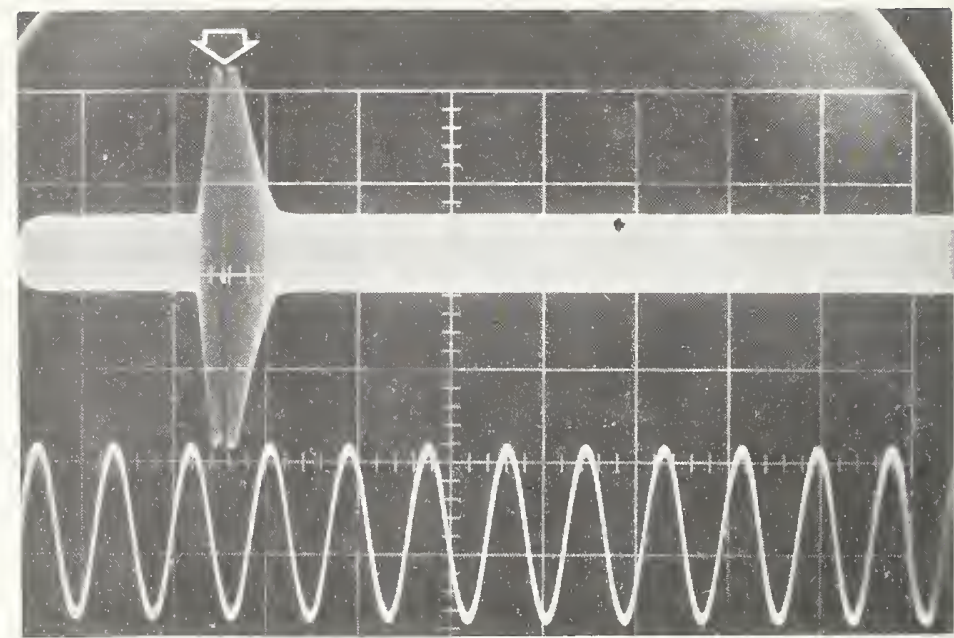

a. The upper bean trace shows the ultrasonic vibration envelope of the motion of the tnnding tool tip, demonstratIng the amplitude variations in a phased burst bonding cycle. Horizontal scale: $5 \mathrm{~ms} / \mathrm{div}$. The portion of this trace indicated by the arrow is expanded on the lower beam trace. Horizontal scale 20 us/div. Vertical scale for the lower trace is about $1 / 2$ that for the upper trace.

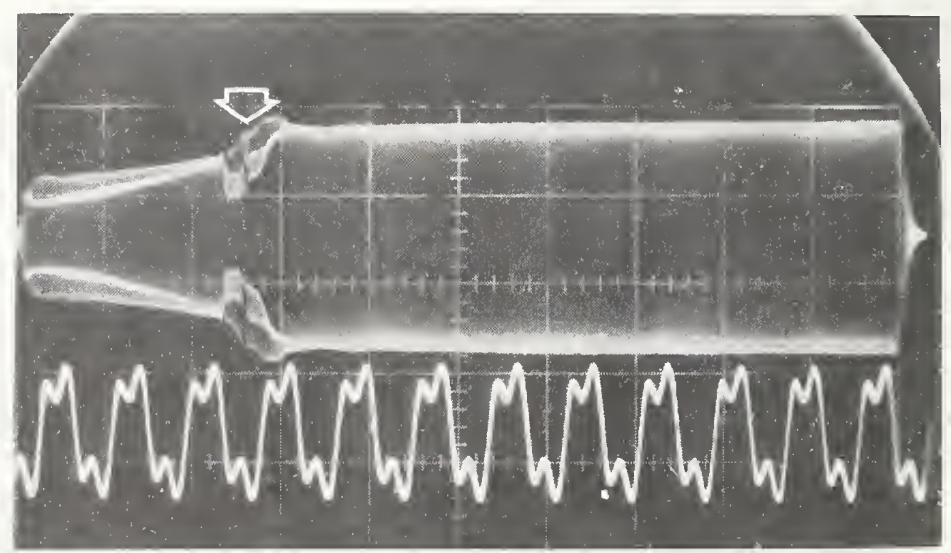

b. Bond formation pattern of a typical adherent bond made with a phased burst bonding cycle. The two traces are as described in Fig. 3-4, except that the vertical scale for the lower trace is about $2 / 3$ that for the urper trace.

Fig. 3-5. Oscilloscope displays of phased burst bonding cycle. 
Earlier studies involving ultrasonic bonding tools (section 2.3) were confined to tools of conventional design made of tungsten carbide. The vibration amplitude of these tools usually remains approximately constant during bonding. Any significant amplitude changes that may occur generally result from the characteristics of the particular transducer or ultrasonic power supply rather than from the tool.

Recently, a tungsten carbide bonding tool, of thin design (sometimes called a 60 deg tool because of the angle that the wire makes with the substrate) and a titanium carbide tool of conventional design have been introduced. Since these two tools represent significant changes from conventional ones, a study was initiated to compare all three types. The data reported here were taken using a single commercial, fixed-tuned, constant-current ultrasonic power supply and matching ferrite-drive transducer. However, additional tests were performed using a nickel magnetostrictive transducer. The vibration amplitude of the tool was measured with a laser interferometer; measurements were also made with a capacitor microphone which had a 4-mil (0.1-mm) diameter tip.

All amplitude measurements were made by synchronizing the laser interferometer at an appropriate time during normal bond formation, generally within the final $5 \mathrm{~ms}$. The actual measurement is made in only about 10 HS. The results were displayed on a double beam oscilloscope and photographed. A typical photograph is shown in Fig. 3-6. The upper trace is the tool tip vibration envelope detected with a capacitor microphone. The arrow near the end of the envelope indicates the time of the laser interferometer measurement. The lower trace is the interferometer wave pattern. Data taken from traces such as this one were used to generate the modal patterns shown in subsequent figures. The synchronization technique was also used to expand part of the vibration amplitude envelope detected by the capacitor microphone in order to reveal any waveform distortions which might be present as shown in subsequent figures.

Laser interferometer measurements were made for the free vibration condition, in which the tool was unloaded, and for a normal bonding load of $25 \mathrm{gf}(0.24 \mathrm{~N})$. Measurements on the unloaded tool, were made with the end of the transducer horn vibrating with an amplitude of approximately 30 $\mu$ in. $(0.76 \mu \mathrm{m})$ peak to peak $(\mathrm{p}-\mathrm{p})$. This is a typical horn amplitude for the normal bonding of 1 -mil $(25-\mu \mathrm{m})$ diameter wire; the modal pattern of the bonding tool is generally such that the vibration amplitude is between 40 and $50 \mu \mathrm{in}$. $(1.0$ and $1.3 \mu \mathrm{m}) \mathrm{p}$-p at the tip. For this particular power supply-transducer combination, the horn amplitude decreased about 25 percent the momunt a bonding load was applied. The changing modal pattern of conventionally designed tools tends to increase the tip amplitude and thus compensate for the decreased horn amplitude. For convenience of display both the loaded and unloaded modal patterns shown in Figs. 3-7 to 3-9 have been normalized to a constant horn amplitude. Thus while the loaded curves may have a higher relative amplitude at the tool tip than the unloaded ones, the actual amplitudes under normal operating conditions may be almost equal. 


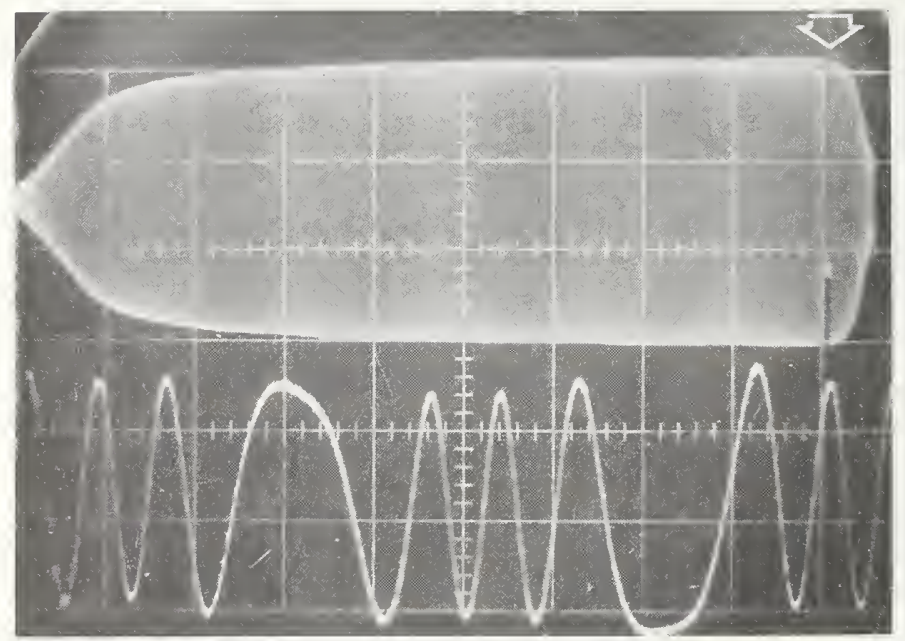

Fig. 3-6. Photograph of typical oscilloscope traces used in measuring modal patterns of ultrasonic bonding tools.

The upper trace is the vibration amplitude envelope as detected by a capacitor microphone; the lower trace is a laser interferometer pattern taken at the synchronization point (arrow). Horizontal scale: $5 \mathrm{~ms} / \mathrm{div}$ (upper trace), $2 \mu \mathrm{s} / \mathrm{div}$ (lower trace). Vertical scale: $20 \mathrm{mV} / \mathrm{div}$. 

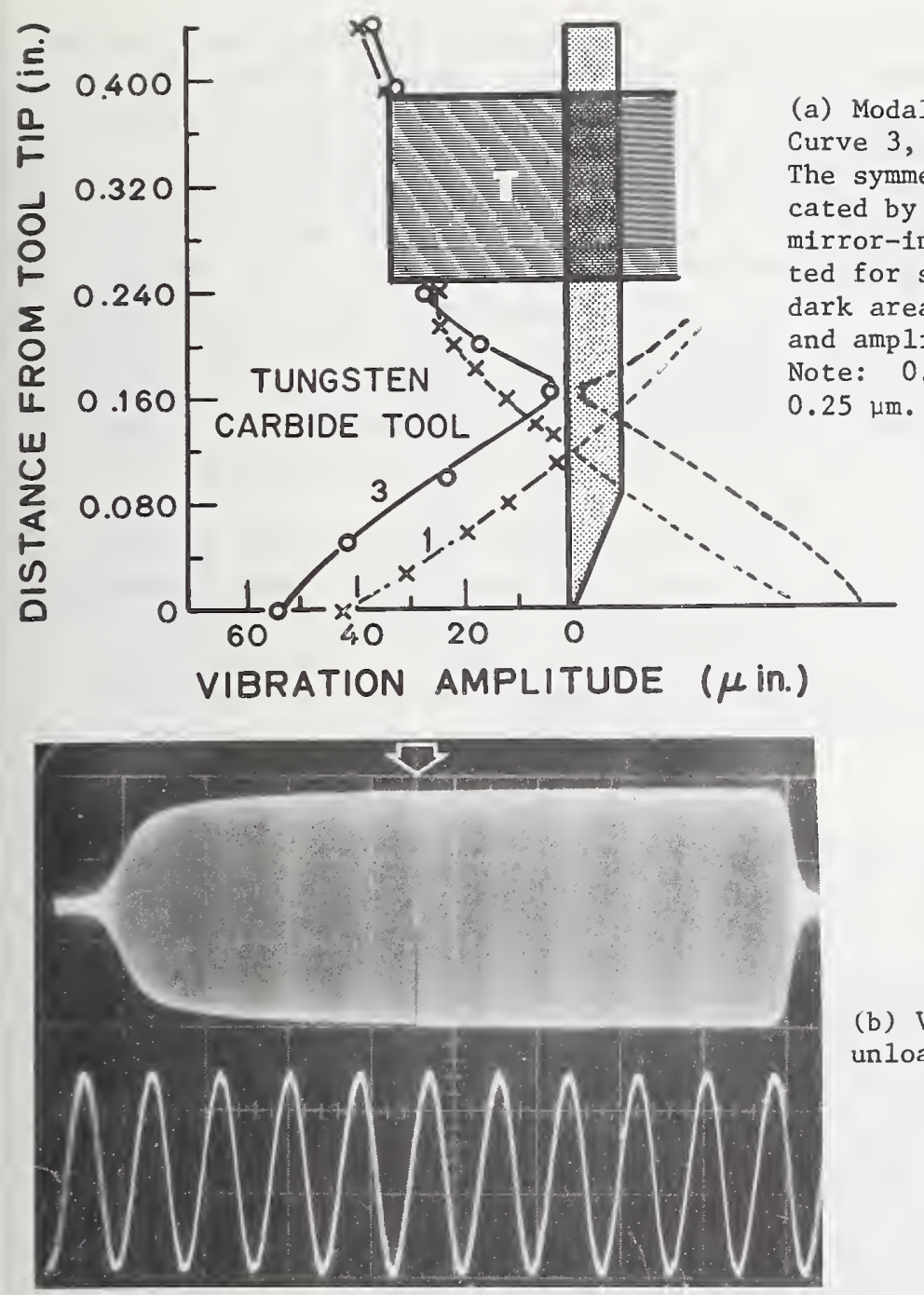

(b) Vibration amplitude patterns for unloaded tool.

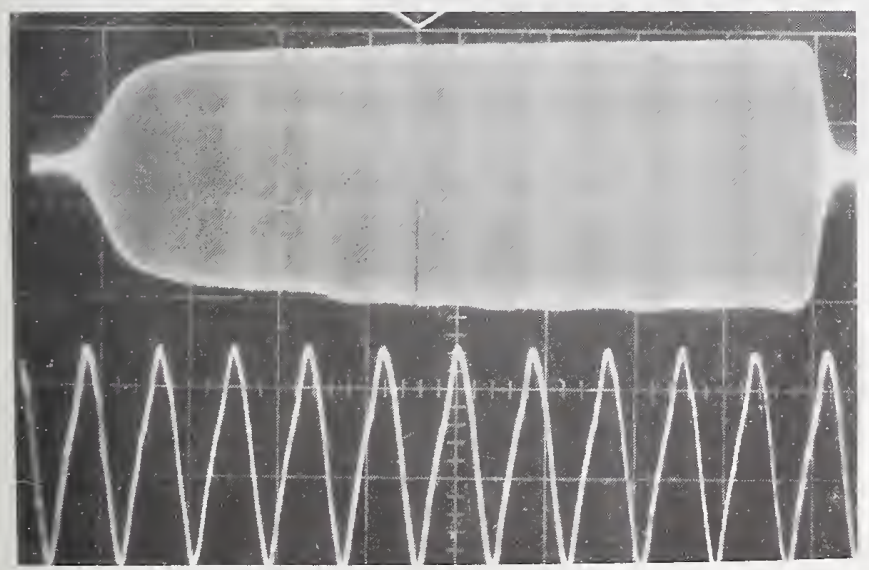

(c) Vibration amplitude patterns for tool during bonding.

Fig. 3-7. Modal patterns and vibration amplitude envelopes of a tungsten carbide bonding tool of conventional design. In (b) and (c) the upper trace is the vibration amplitude envelope, measured $35 \mathrm{mils}(0.9 \mathrm{~mm})$ above the tool tip; the lower trace is the amplitude waveform expanded at the time indicated by the arrow. Horizontal scales: $10 \mathrm{~ms} / \mathrm{div}$ (upper trace); $20 \mu \mathrm{s} / \mathrm{div}$ (1ower trace). Vertical scales: $20 \mathrm{mV} / \mathrm{div}$. 
The modal patterns and vibration amplitude envelopes of a tungsten carbide tool of conventional design are shown in Fig. 3-7. Loaded nodes are se1dom as sharp or as complete as the unloaded ones as can be seen in the modal patterns of Fig. 3-7a. The nodal position during bonding is a function of the mechanical loading of the tool tip during bonding; this is primarily a function of the wire deformation, which was approximately 1.6 for the case shown. A smaller deformation, such as 1.2, may result in a nodal rise of only 20 to $25 \mathrm{mils}(0.50$ to $0.64 \mathrm{~mm})$ from the unloaded position. This tool showed no significant change in tip amplitude during bonding, but such changes would be expected to become more significant as the bonding force is increased for bonding larger diameter wire. Dushkes [11] has reported reduction of tip amplitude under such conditions.

The upper trace in Fig. $3-7 b$ is the vibration amplitude envelope of the unloaded tool as detected by the capacitor microphone placed approximately $35 \mathrm{mils}(0.9 \mathrm{~mm})$ above the tool tip. The lower undistorted sine wave was expanded from the envelope at the position of the arrow. These traces are typical of those observed under unloaded conditions on all the tools in the present study; unloaded traces are not pictured for subsequent tools.

The traces in Fig. 3-7c were taken from the same tool during the formation of a good metallurgical weld. Although the envelope (upper trace) is nearly the same as that of the unloaded case, some waveshape distortion is apparent in the expanded waveform (lower trace). The degree of distortion can vary considerably if a different transducer or a bonding tool of similar design and material but from a different manufacturer is used. Some tools of conventional design show no waveshape distortion during bonding.

The modal patterns of a titanium carbide tool of conventional design under both loaded and unloaded conditions are shown in Fig. 3-8a. Titanium carbide has a much lower density and is more flexible than tungsten carbide. The nodal position is approximately $20 \mathrm{mils}(0.5 \mathrm{~mm})$ above that of a tungsten carbide tool of the same geometry (see Fig. 3-7a). As a result, the vibration amplitude of the unloaded tool tip is about 20 percent greater for the same transducer drive. As soon as the tool presses against the wire with the normal $25 \mathrm{gf}(0.24 \mathrm{~N})$ bonding force, the node becomes blurred and almost ceases to exist. However, except for this difference, the modal pattern has the same general appearance as that of the tungsten carbide tool during bonding. The traces in Fig. 3-8b were taken on the titanium carbide tool under the same loading conditions as the traces on the tungsten carbide tool shown in Fig. 3-7c. Although the envelope (upper trace) is nearly identical with that of the unloaded tungsten carbide tool (Fig. 3-7b), some distortion is evident in the expanded waveform (lower trace). 


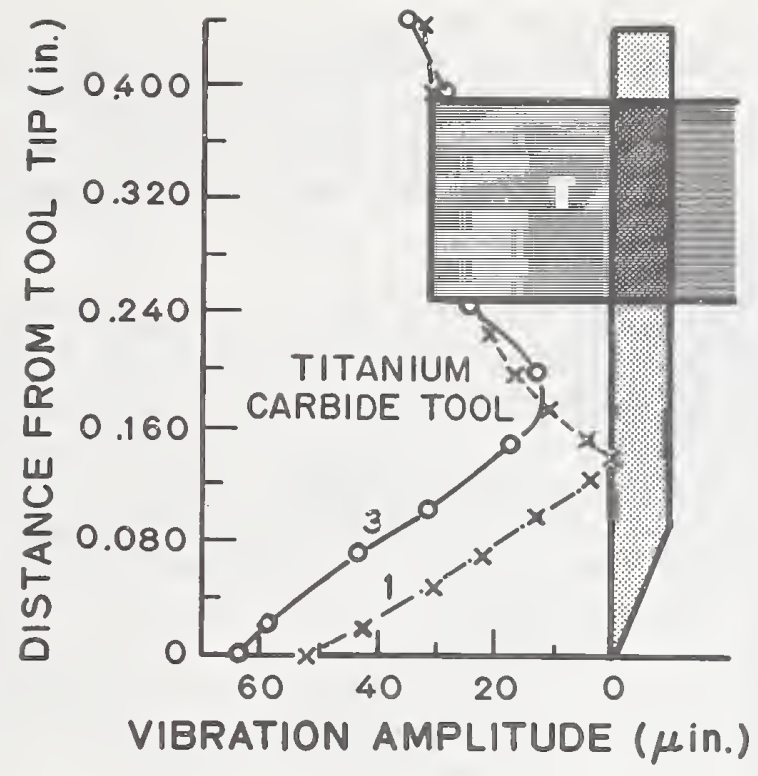

(a) Modal patterns: Curve 1, unloaded; Curve 3,5 ms before end of bonding cycle. Note: $0.010 \mathrm{in}$. $\approx 0.25 \mathrm{~mm}$ : $10 \mu \mathrm{in} . \approx 0.25 \mu \mathrm{m}$.

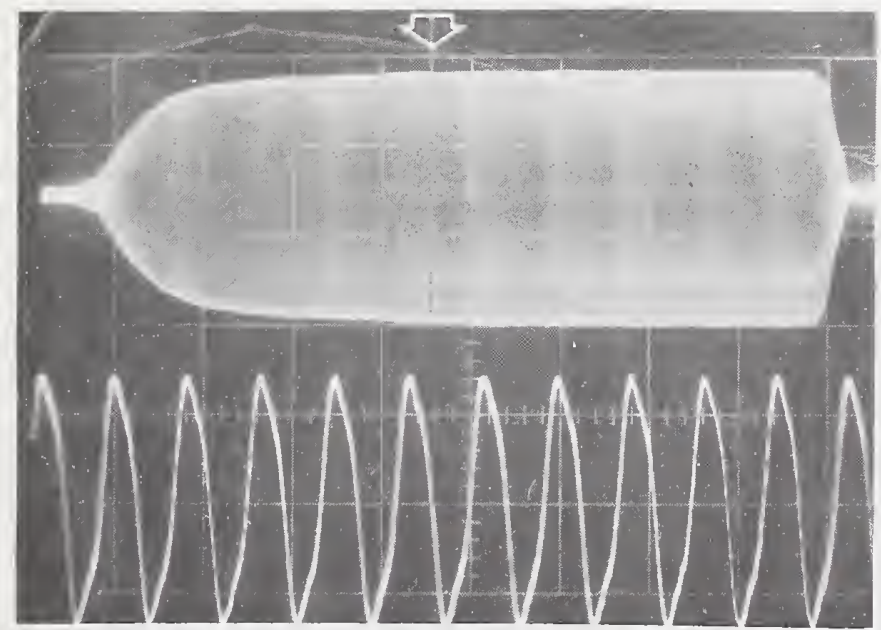

(b) Vibration amplitude patterns for tool during bonding.

Fig. 3-8. Modal patterns and vibration amplitude envelope of a titanium carbide bonding tool of conventional design. In (b) the upper trace is the vibration amplitude envelope, measured $35 \mathrm{mils}(0.9 \mathrm{~mm})$ above the tool tip; the lower trace is the amplitude waveform expanded at the time indicated by the arrow. Horizontal scales: $10 \mathrm{~ms} / \mathrm{div}$ (upper trace); $20 \mu \mathrm{s} / \mathrm{div}$ (lower trace). Vertical scales: $20 \mathrm{mV} / \mathrm{div}$. 
Characteristics of the tungsten carbide tool of a thin-line design are shown in Fig. 3-9. This tool is intended to fit into very small spaces and also to be used in forward, or post-to-die, bonding. Preliminary studies suggested that this tool had rather unusual operating characteristics so the study of loading effects was more thorough than that undertaken for either of the other tools.

The laser interferometer was synchronized to measure the vibration amplitude $7 \mathrm{~ms}$ after the initiation of bonding and again $5 \mathrm{~ms}$ before the end of the bonding cycle for each bond made. Thus it was possible to obtain two different modal patterns for the tool during actual bonding in addition to the unloaded modal pattern as shown in Fig. 3-9a. At each position on the tool, both data points obtained under loaded conditions were obtained during the same bonding cycle. Although severe loading of the tip, termed "lug down" and believed to contribute to erratic bonding by Dushkes [11], is apparent at the beginning of the cycle, it is especially pronounced at the end of the cycle. An interesting feature of this tool is that the final tool tip vibration amplitude is less than two-thirds of the initial amplitude.

The vibration amplitude envelope at a position approximately $35 \mathrm{mils}$ ( 0.9 $\mathrm{mm}$ ) above the tool tip near the knee of the loaded modal pattern is shown in Fig. 3-9b. This envelope is asymmetrical and its amplitude increases with time in a way similar to the envelopes shown in Figs. 3-7c and 3-8b; this is in agreement with the modal pattern in Fig. 3-9a. The distortion in the expanded waveshape in the lower trace also exhibits asymmetry.

The vibration amplitude envelope and the distortion in the expanded waveshape at a position within $5 \mathrm{mils}(0.13 \mathrm{~mm})$ of the bottom of the tool exhibit substantially different characteristics from those observed higher on the tool. These characteristics change from bond to bond. Traces for two successive bonds are shown in Figs. 3-9c and 3-9d. Both bonds resulted in good metallurgical welds and were visually identical. These traces, taken on normal, $45 \mathrm{~ms}$, bonding cycles were similar to others at this position taken on the longer ( $\sim 75 \mathrm{~ms}$ ) bonding cycle. The initial peak-to-peak vibration amplitudes of the tool tip during these and other bonds ranged from 30 to 55 percent higher than the final amplitude; it should be noted that ultrasonic power is proportional to the square of the vibration amplitude. This amplitude variation was not observed in either of the other tools studied; in those cases, changing either the microphone position or the bonding time resulted in no significant change in the envelope or the distortion of the expanded waveshape.

Vibration amplitude characteristics of the bonding tool tip during attempted bond formation over a void under the pad to obtain a poor metallurgical weld are shown in Fig. 3-9e. The appearance of the vibration amplitude envelope is more characteristic of a position higher on the tool (see Fig. 3-9b). Distortion of the expanded waveshape is the most severe observed in these tests. 


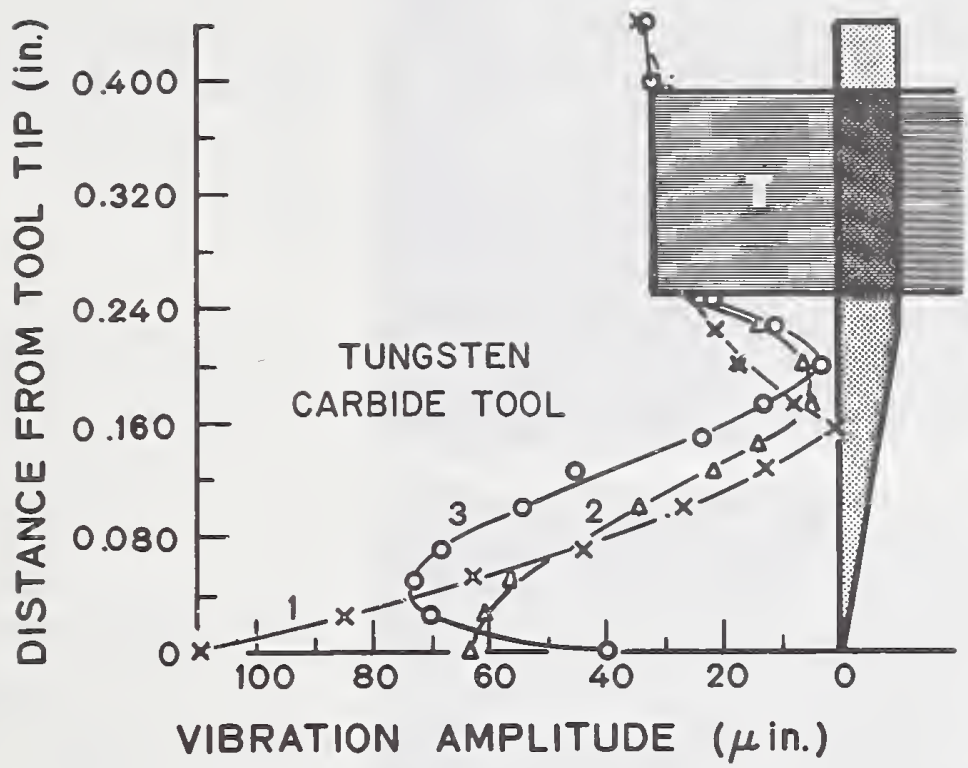

Fig. 3-9. (a) Modal patterns: Curve 1, unloaded; Curve 2, 7 ms after beginning of bond-

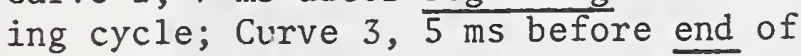
bonding. Each point on the loaded curves ( 2 and 3 ) are an average obtained from 3 bonds.

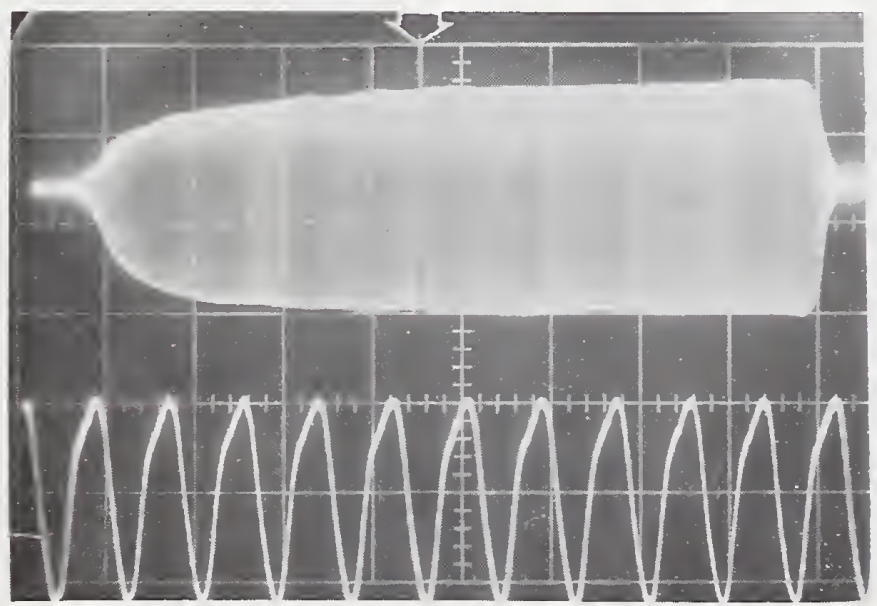

Fig. 3-9 (b) Vibration amplitude patterns, measured $35 \mathrm{mils}(0.9 \mathrm{~mm})$ above the tool tip, for a bonding cycle which resulted in a good metallurgical weld. 

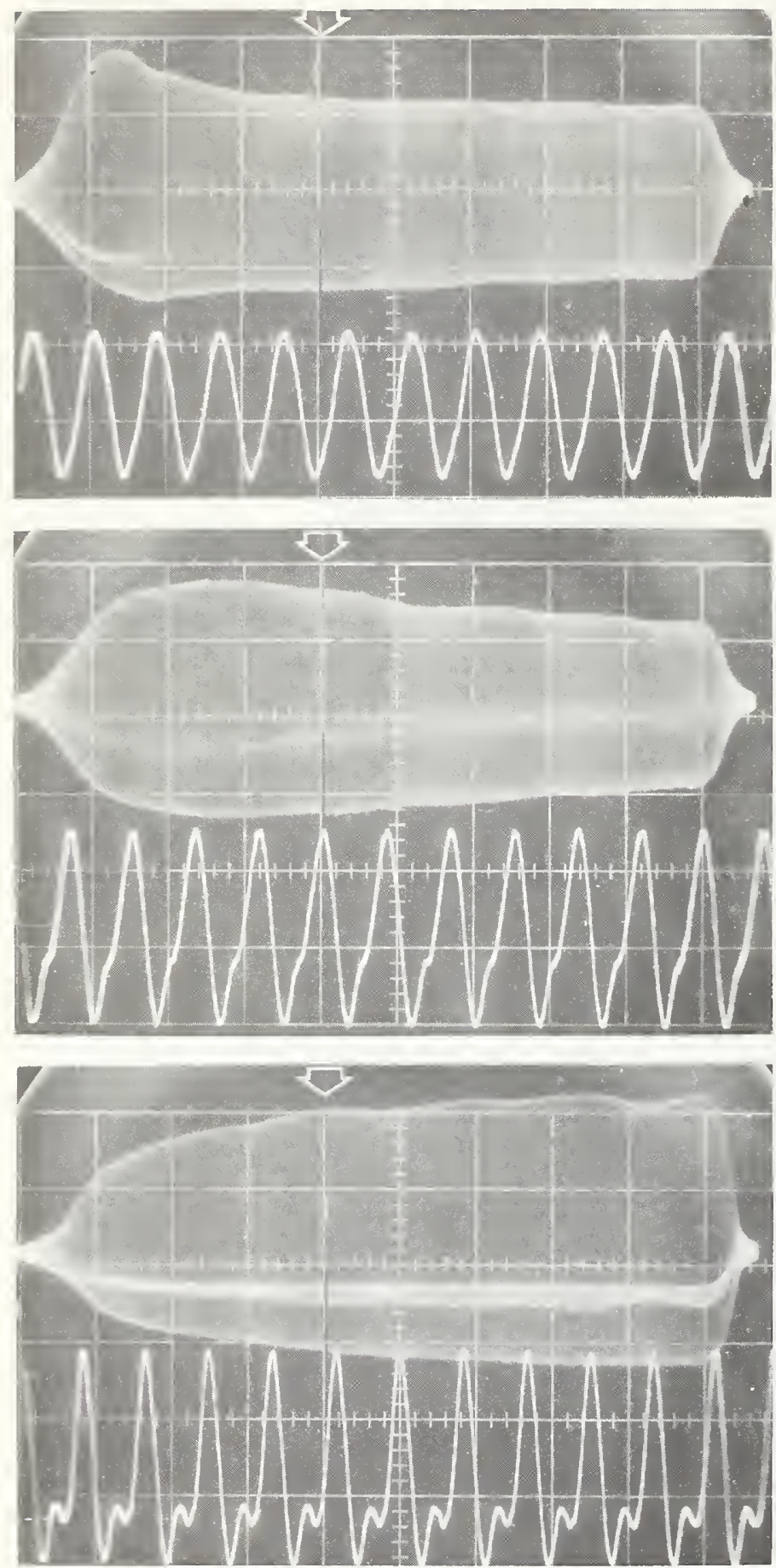

(c) Vibration amplitude patterns, measured at the tool tip, during first of two successive bonding cycles which resulted in good metallurgical welds.

(d) Vibration amplitude patterns, measured at the tool tip, during second of two successive bonding cycles which resulted in good metallurgical welds.

(e) Vibration amplitude patterns, measured at the tool tip, during formation of a poor bond. This bond was intentionally made above a void under the bonding pad.

Fig. 3-9. Modal patterns and vibration amplitude envelopes of a tungsten carbide tool of thin-line design. In (b)-(e) the upper trace is the vibration amplitude envelope; the lower trace is the amplitude waveform expanded at the time indicated by the arrow. Horizontal scales: $10 \mathrm{~ms} / \mathrm{div}$ (upper trace (b) ; $5 \mathrm{~ms} / \mathrm{div}$ (upper trace (c)-(e)); $20 \mathrm{\mu s} / \mathrm{div}$ (lower trace (b)-(e)). Vertical scales: $20 \mathrm{mV} / \mathrm{div}((\mathrm{b})-(\mathrm{e}))$. 
These results suggest that the thin-line tool apparently has some ability to compensate for slight differences in the substrate or wire condition. On the basis of 1 imited tests, not described in detail, it appears that this tool requires careful control of the extension of the tool below the transducer horn in order to achieve the most reproducible results.

(G. G. Harman)

\subsubsection{Long ULtrasonic Bonding Tools}

The same measurement techniques and equipment described above were used to study the vibration modes of long [0.828 in. (20 mm)] bonding tools. Only two tools were studied; one was made of tungsten carbide (WC) and the other was of titanium carbide, both being of conventional design. The thin, $60^{\circ}$ tool design was not available in the 0.828 in. 1ength. As above, the modal patterns were normalized to the same $30 \mu \mathrm{in} .(0.76 \mu \mathrm{m})$ $\mathrm{p}-\mathrm{p}$ transducer horn amplitude for clearest display, and the data obtained during bonding was taken within the last $5 \mathrm{~ms}$ of the bonding cycle.

Fig. 3-10 gives the modal patterns of a long tungsten carbide bonding tool set to a manufacturers recommended extension of 0.555 in (14 mm) below the horn. The tool extension above the horn approaches a quarter wave length and thus its vibration amplitude is several times that of the bottom. Besides the double node the major difference between this long tool and the comparable short one is the small shift in nodal position during bonding. The nodal rise observed with this long tool was only 0.015 to $0.020 \mathrm{in}$. ( 0.38 to $0.50 \mathrm{~mm})$ resulting in a negligible vibration amplitude increases at the tool tip.

Fig. 3-11 gives the modal pattern of a long titanium carbide bonding tool. The physical characteristics of this material are quite different from those of tungsten carbide, and the nodes shift more under load. The tool extension above the transducer, although the same length as that of Fig. 3-10, is no longer a mechanical quarter wavelength so its amplitude is similar to that of the tool tip. The upward nodal shift [approx. 0.03 in. $(0.76 \mathrm{~mm})]$ during bonding is not quite as great as that for the comparable short tool [approx. $0.05 \mathrm{in} .(0.13 \mathrm{~mm})]$, but it is greater than that of the WC tool in Fig. 3-10. The loaded node becomes broad and imprecise as on the short tool, but again to a lesser extent.

(G. G. Harman)

\subsection{Studies of the UTtrasonic Bonding Mechansim}

\subsubsection{Bond Lift-off: Laboratomy Experiments}

By studying the bonding pad after a bond has been intentionally lifted or peeled off, the "footprint" pattern of bond formation can be seen. The threshold of welding can be observed by decreasing either the power or time in the bonding schedule to a point where essentially no bonding has taken place. This experiment was performed for a number of first and second bonds. Examples are shown in Fig. 3-12. (Bonding machine movement, during bonding, Section 2.2.1, is apparent in the lift-off pattern of Fig. 3-12d.) 


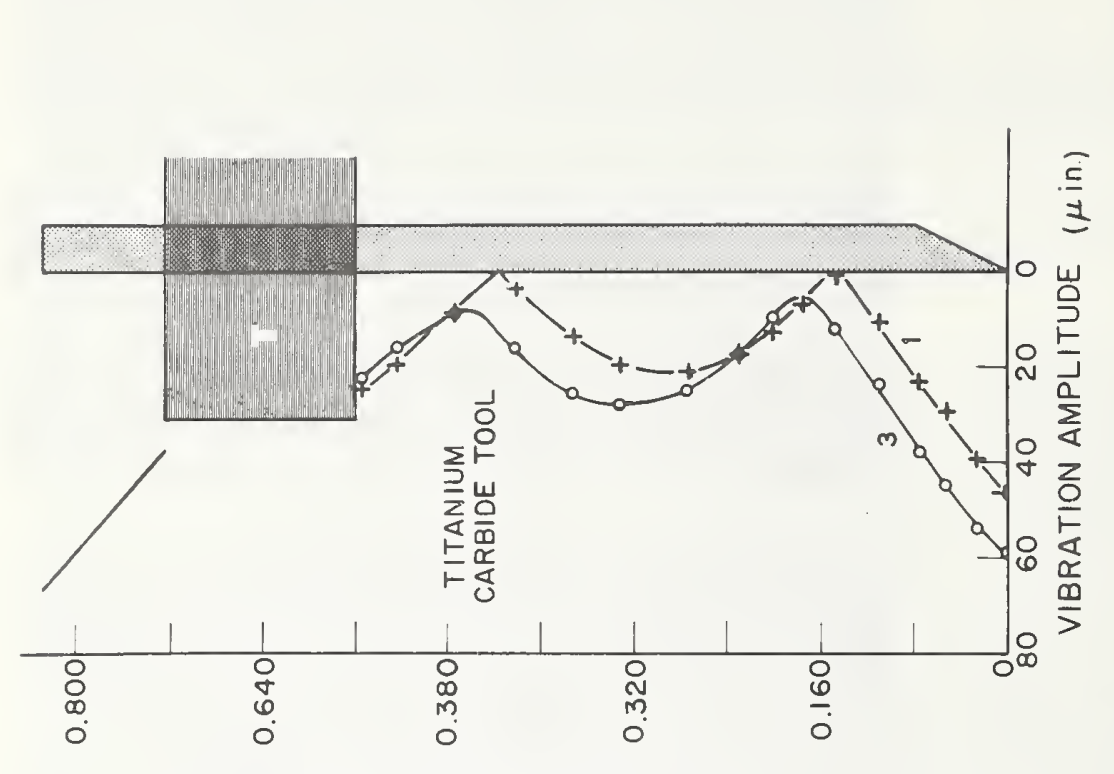

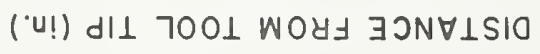

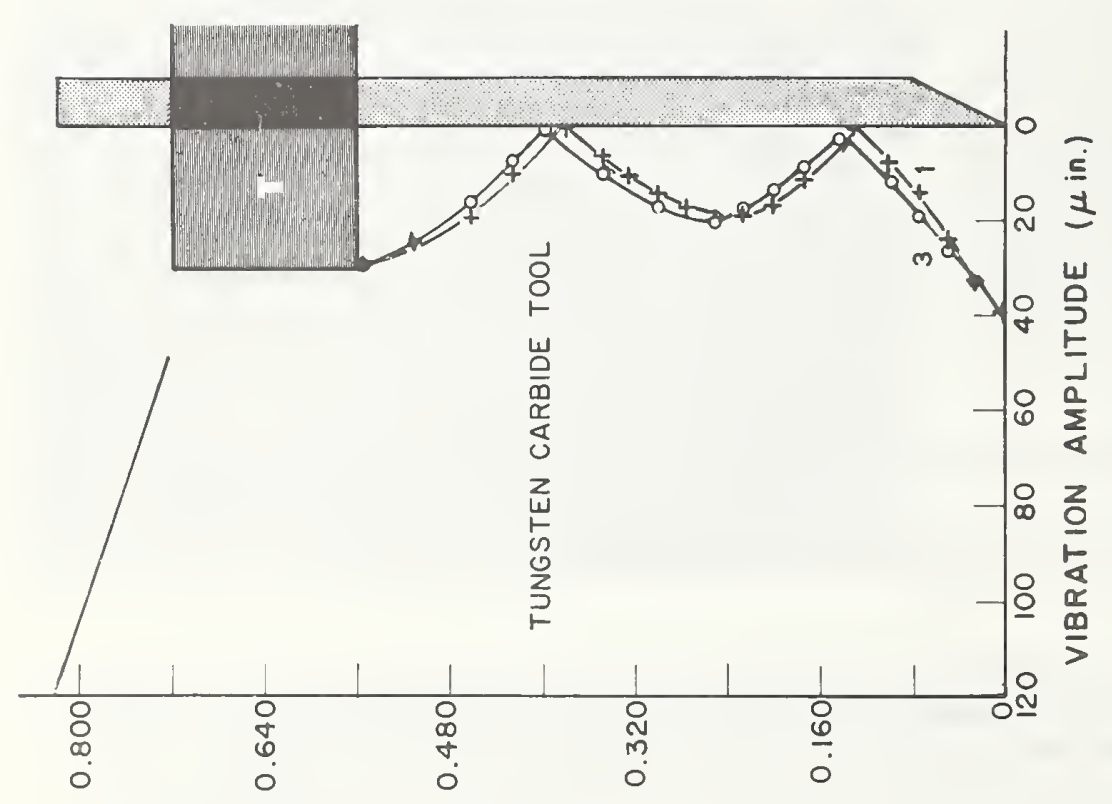

(u!) dlI $700 \perp$ WOY $\exists 3 N \forall \perp S I O$

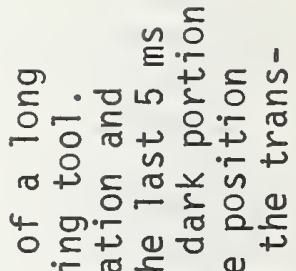

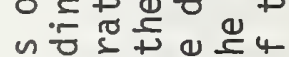

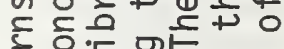

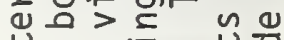
† ब व ช 0 \& 0 ब ․ㄷำ 一 ᄂ

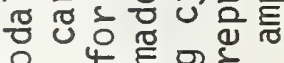
은

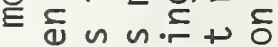

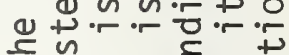
Е 1

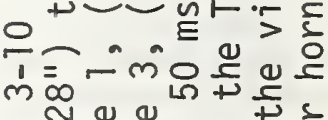
०ळ

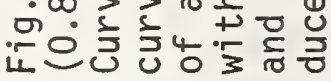



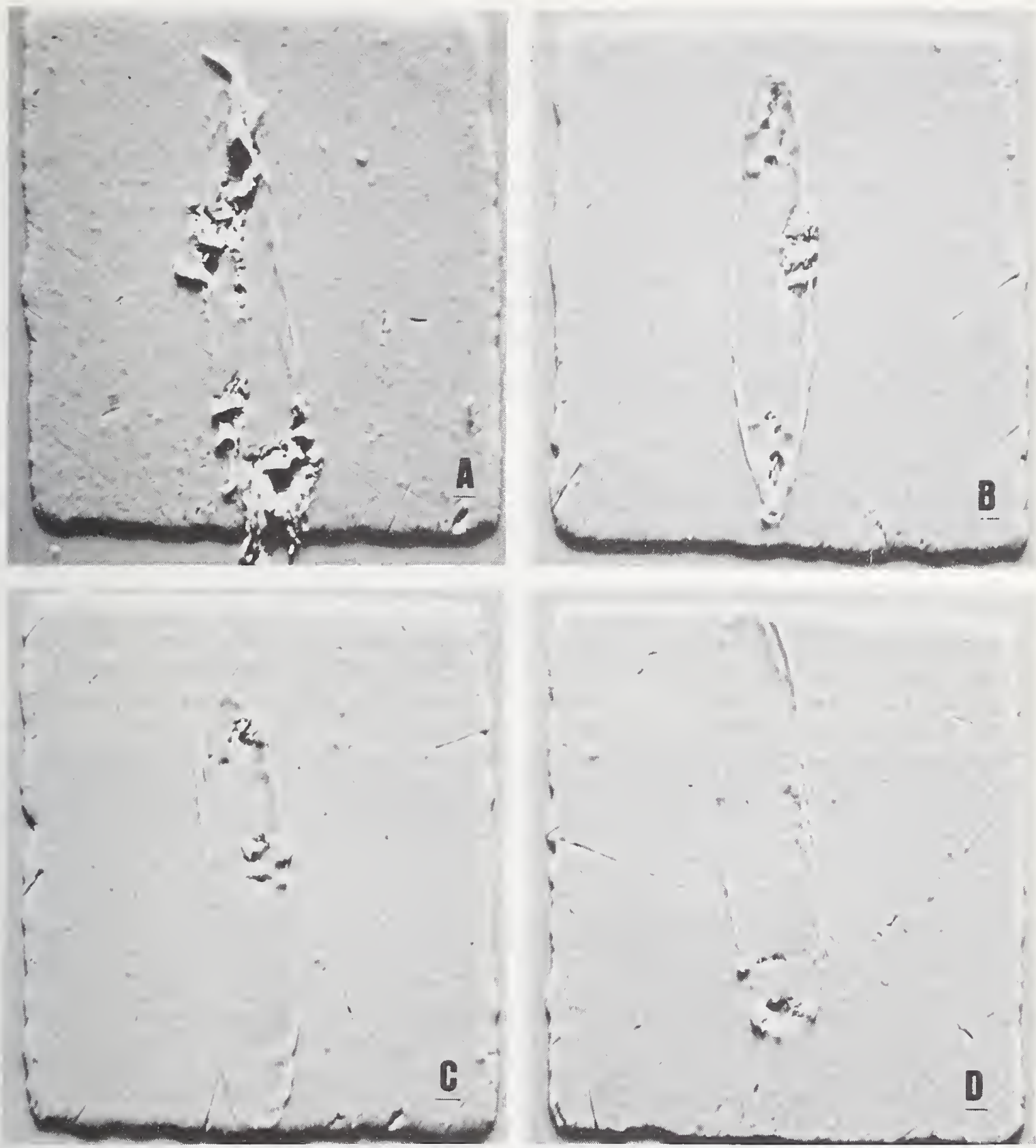

Fig. 3-12. SEM photomicrographs (460X) of bond lift-off patterns for first bonds made at different time settings and constant power setting. The time is longest for case (a) and is decreased to the shortest value for case (d). Settings used in case (a) result in a bond which will adhere to the pad; the shorter time settings do not produce an adherent bond. The scratches on the bonding pads (which result from a cleaning process no longer in use) are smoothed,out by the impact of the tool compressing the wire against the pads before ultrasonic energy is applied. Note also the apparent movement during bonding exhibited in case (d). This results from mechanical vibration in the bonding machine. 
There are general characteristics of bond patterns which can be observed. From cases in which very low power or short time was used, such as that shown in Fig. 3-12d, it is apparent that much of the wire deformation occurs before welding begins and is due to the impact of the tool against the wire. When welding begins, it starts at tiny isolated points, generally around the perimeter, which are seldom located in the same position for successive bonds. Occasionally the weld will start in entirely different sections of the bond. An example of two consecutive second bonds made with the same bonding conditions is shown in Fig. 3-13. As the power or time is increased, the weld extends to other areas. The center is the last portion to weld. Even in some very strong bonds, the center area may remain unbonded. The exact way the weld progresses apparently depends on the bonding machine design (one or two nodes on the bonding tool, the angle the tool makes with respect to the substrate, the bonding schedule, etc.).

A photomicrograph of an ultrasonic aluminum wire-bond 1 ift-off pattern that was obtained by carefully peeling off a bond is shown in Fig. 3-14. It reveals the pattern of a typical ultrasonic weld made with one type of bonding machine. The center portion of the bond is generally not welded. It is estimated that much of the strength of such bonds is due to the relatively large welded area at the heel and toe of the bond. The photomicrograph of Fig. 3-15 displays the same features, but shows both the partially removed wire and the pad. Studies with different bonding schedules or with different makes of bonding machines have indicated that in some bonds, welding may extend all the way through the center. There is no apparent disadvantage in leaving a small unwelded area in the center. Whether an unbonded area exists or not, the wire can be strongly bonded to the pad so that its failure mode in a pull test will typically be breakage at a bond heel.

(K. O. Leedy)

\subsubsection{Bond Lift-Off: Commercial Devices}

[560] Bond lift-off patterns have been observed in devices intended for highreliability applications. One example that reveals the absence of welding in the center of the bond is shown in Fig. 3-16. The original bond apparently had lifted off and a second bond was made to the pad. A possible cause for the 1 ift off appears to be an abnormal front-to-back motion of the bonding tool with respect to the work stage that presumably occurred near the end of the bonding cycle.

Another example of a lift-off from a different type of device intended for high-reliability application is shown in Fig. 3-17. Sideways motion of the bonding tool relative to the work stage is evident in the lift-off pattern. Its smooth appearance indicates little welding. This combined with the very small deformation of the rebonded wire suggests that the bonding schedule used for these bonds was in the underbonded region that produces statistically unreliable bonds. 

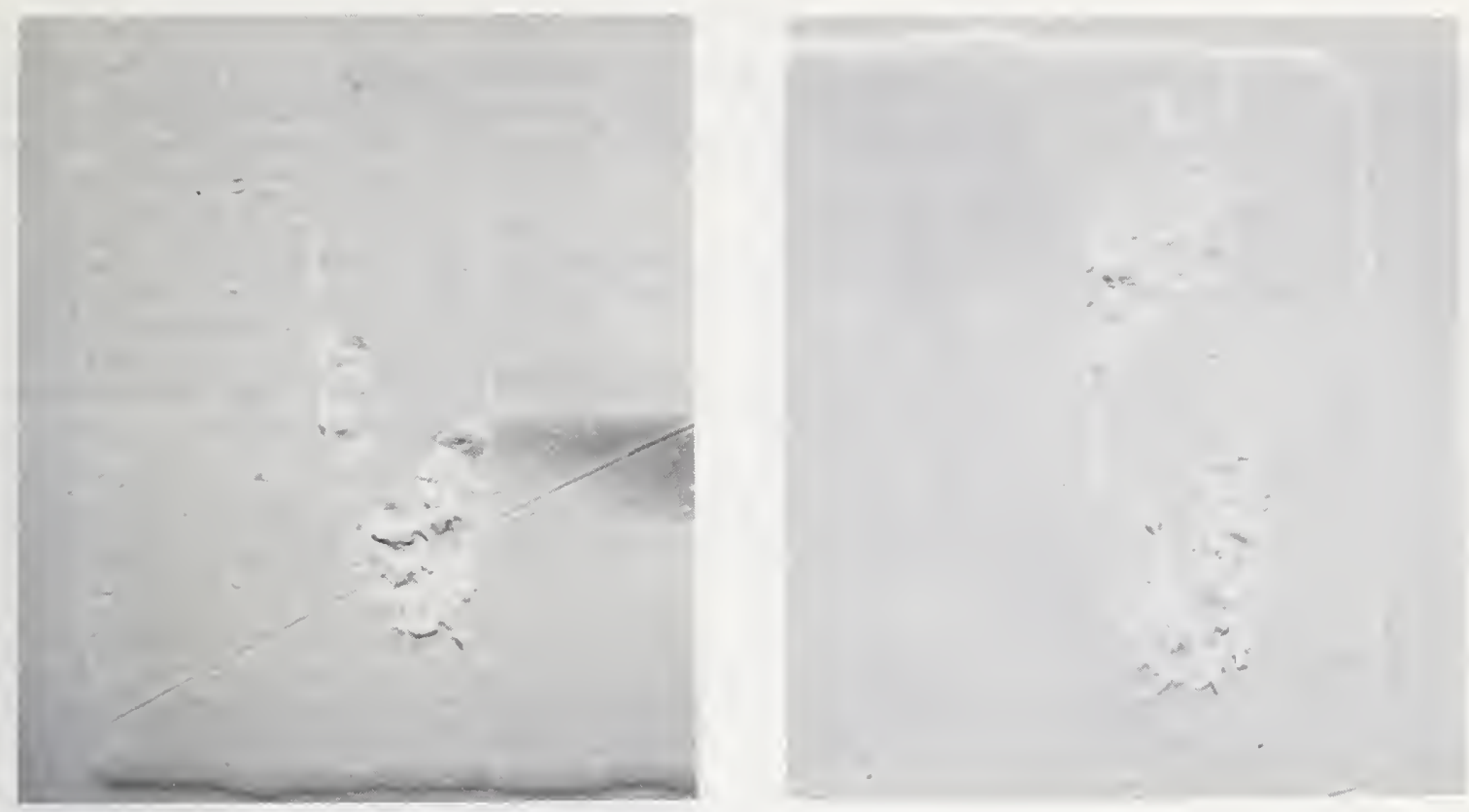

Fig. 3-13. SEM photomicrographs (600X), of bond 1ift-off patterns for two second bonds made consecutively at identical time and power settings.

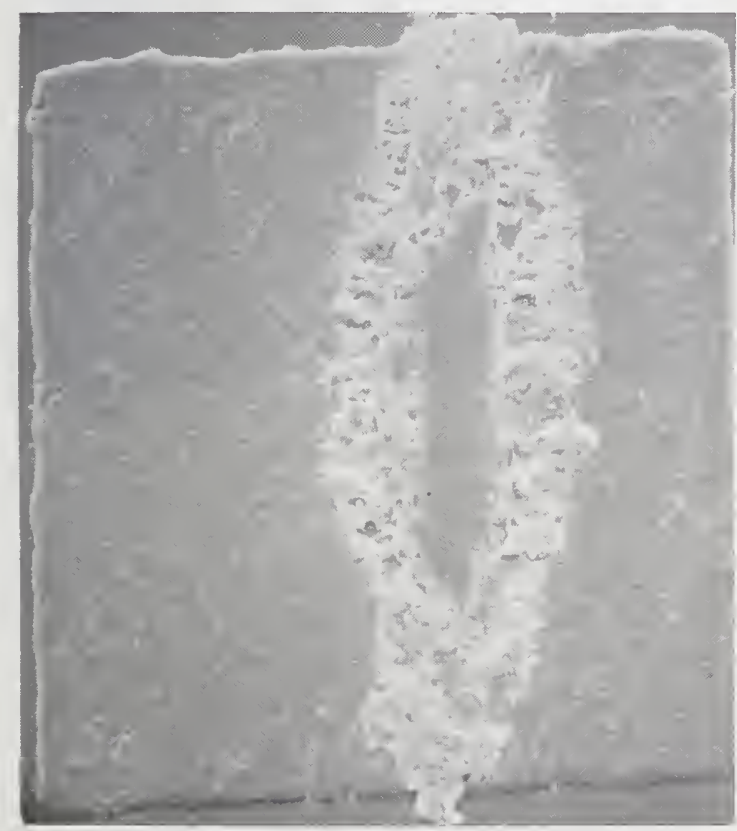

Fig. 3-14. SEM photomicrograph of the 1ift-off pattern of a normal bond made under laboratory conditions. The pattern, which clearly reveals the unwelded center portion of the bonded region, was exposed by carefully peeling back the bond. Magnification: $510 \mathrm{x}$.

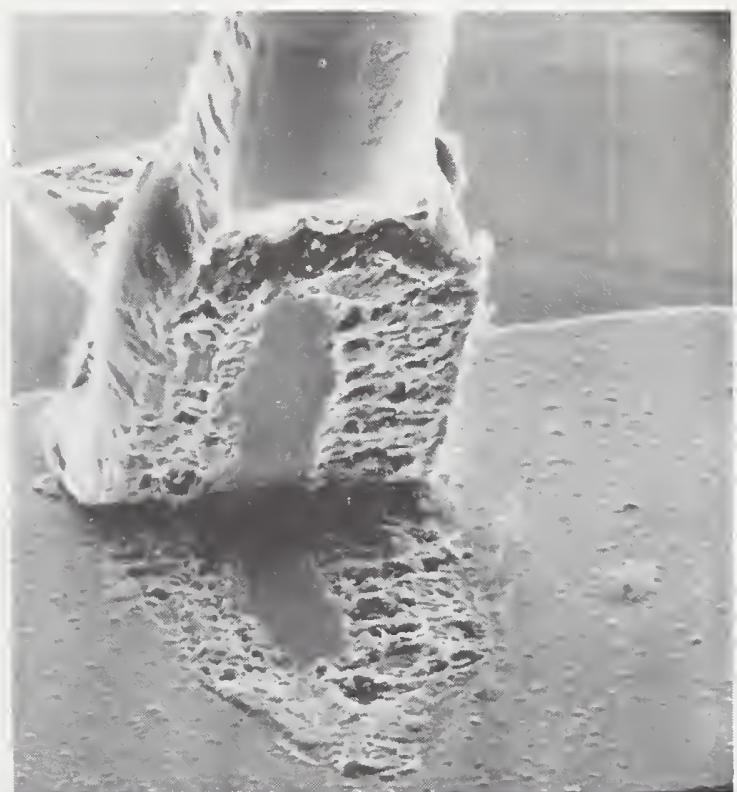

Fig. 3-15. SEM photomicrograph of the lift-off pattern of a partially removed bond made under laboratory conditions. A similar pattern can be observed on both the wire and the pad. Again the center portion of the bonded region is not welded. Magnification: $900 \mathrm{x}$. 


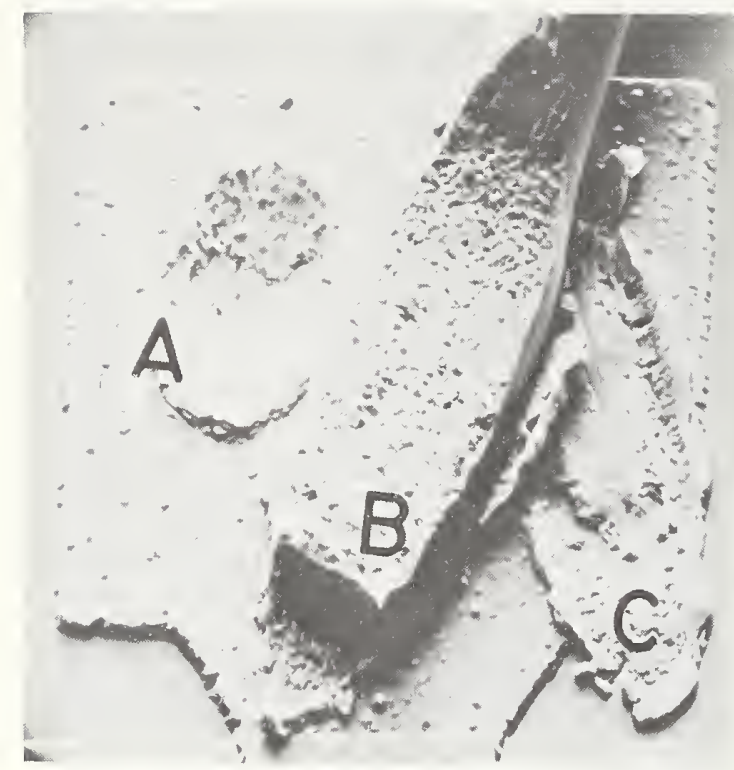

Fig. 3-17 SEM photomicrograph of a bond and bonding pad from a comercial device that reveals sideways bonding tool motion. The device was intended for high rellability application. The orfginal bond (A) lifted off because of underbonding. The small deformation of the rebonded wire (B) suggests that the bonding schedule used in making this device was unchanged after the fallure at (A). An electrical test probe mark is also visible (C). Magnification: $540 \mathrm{x}$.

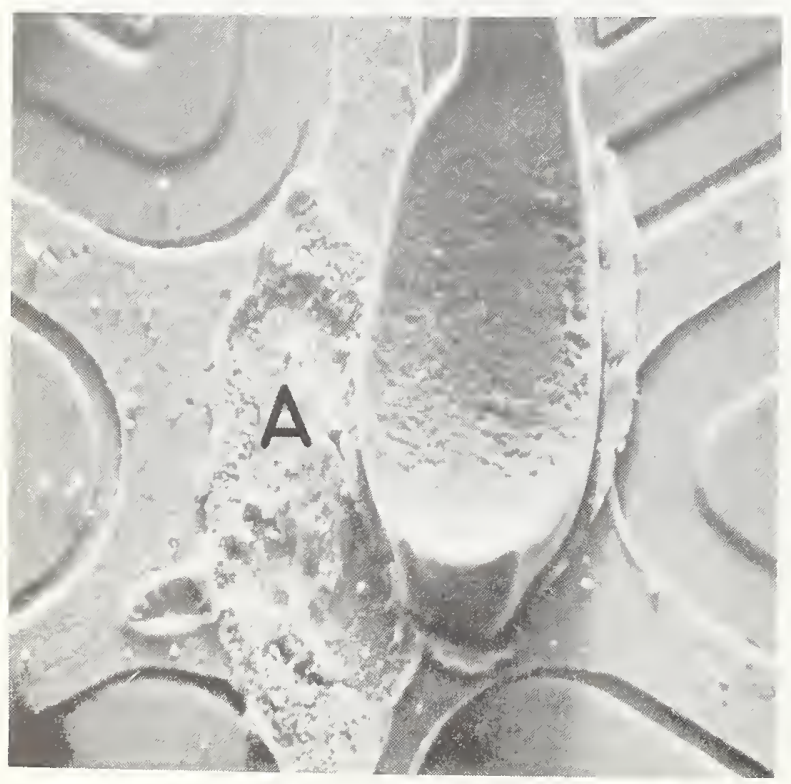

Fig. 3-16. SEM photomicrograph of a bond and bonding pad from a commercial device that reveals the typical unwelded area noted in bonds made under laboratory conditions. The device was intended for high reliability application. The photomicrograph shows a probe mark (A) and rebond (B). The original bond (C) apparently lifted off because it was weakened by forward motion of the bonding tool. Magnification: $520 \mathrm{x}$.

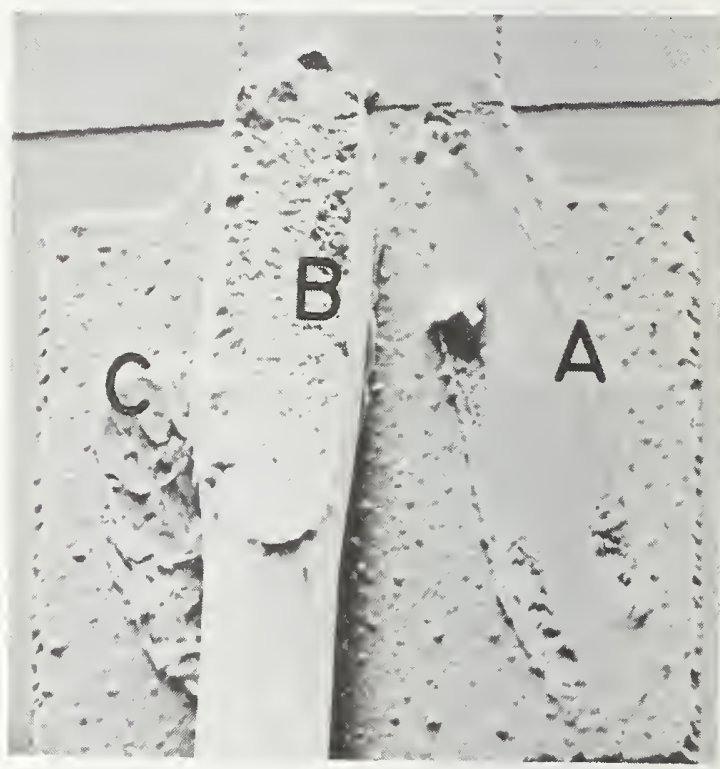

Fig. 3-18. SEM photomicrograph of a bond and $\rightarrow$ bonding pad from a commercial device that has a very small bonding pad. The device was intended for high reliability application. The lift off of the original bond (A) may have been caused by overbonding or poor metallization. Magnification: $475 \mathrm{x}$. 
A lift-off pattern that shows evidence of overbonding (such as excessive power or time) or poor metallization is shown in Fig. 3-18. The difficulties of rebonding to a very small pad without causing an electrical short are also obvious from this example.

(K. 0. Leedy)

\subsubsection{Ultrasonic Bonding Mechanism}

In order to gain a better understanding of the mechanisms involved in ultrasonic wire bonding, experiments have been performed using some of the techniques previously developed in this Program, such as examination of bond lift-off patterns with the SEM and measurement of the bonding tool vibration amplitude with the capacitor microphone.

Preliminary results of this work were reported [12]; and a more complete paper has been recently published [13]. Reprints of the paper are available from the authors. Its conclusions were the following:

1. The bonding process is closely related to ultrasonic softening of the weldments. Evidence is presented to suggest that heat is a by-product of the softening process, not the contrary as has been generally believed, and the amount of heat generated alone is insufficient to account for the welding of aluminum.

2. During the first $10 \mathrm{~ms}$ or so after ultrasonic energy is applied, little ultrasonic energy is coupled into the wire and bonding pad system as evidenced by the degree of loading observed in the tool. However, it is during this period that the first metallurgical welds between the wire and the substrate are observed to develop, thus permitting subsequently increased coupling of energy into the system. After 10 to $15 \mathrm{~ms}$, a slower growth of the larger welded area then progresses as the wire deforms.

3. The deformation of the wire as it is pushed against the substrate, not ultrasonic vibration or sliding of the wire, breaks up and sweeps aside the oxide and other interfacial contaminants exposing clean metal surfaces that are then able to form metallurgical welds.

4. It is not necessary for the tool to grip the wire and slide it back and forth across the pad to achieve bonding. Instead for most microelectronic bonding, the ultrasonic energy is coupled into the system by the tool moving across the top of the wire at all stages of bond formation. Tool-to-wire coupling takes place by a series of microwelds that are repeatedly made and broken as the tool moves back and forth across the wire surface.

(G. G. Harman and K. O. Leedy) 


\subsection{Bond Failure Resulting from Device Power Cycling}

\subsubsection{Theoretical Results}

[598] In some applications, such as telemetry, transistors are operated within their power ratings but are subjected to cyclical applications of power. Many transistors have been observed to fail under such conditions of thermal cycling at slow rates ( 1 or more minutes per cycle). This failure has been traced to aluminum interconnection wire fatigue resulting from repeated thermal-expansion-induced wire flexing [14, 15]. A study was made of the wire bond flexure resulting from such cycling of 2 N2222 (500 mW, $50 \mathrm{~mA})$ transistors. This low current does not produce significant Joule heating of the lead wires. * However, the chip may reach $180^{\circ} \mathrm{C}$ and cause considerable thermal expansion. For the thermal expansion calculation, the chip was assumed to be the sole source of heat. The simple analysis was made specifically for the 1-mil (25- $\mu \mathrm{m})$ diameter, aluminum wire bonded to a particular transistor in a T0-18 can, Fig. 4-1, however, it is generally applicable to other devices with similar current and power ratings.

It was found that the changes in loop height and the angle of the wire at the bond heel were approximately proportional to the dissipated power and inversely proportional to the equilibrium loop height. Since wire flexing is reduced as the loop height is increased, failures due to wire flexing are minimized if a large loop height is used.

[733] For a more complete solution of the wire flexing problem the aluminum bonding wire was modeled to compute its temperature distribution by means of the TRUMP program [16]. The model was idealized in that the wire and post were assumed collinear with a butt joint type of bond. For junction temperatures up to $180^{\circ} \mathrm{C}$, corresponding to the rated power input of 500 mi, it was found that the temperature distribution was linear within the wire and that the temperature drop along the wire was two-thirds the junction-to-case temperature difference whether the case was heat sinked or not. Practically all of the remaining third of the temperature drop occurred in the post very close to the joint. The physical interpretation of the linear temperature distribution is that heat transfer occurs predominantly by conduction. Radiation is less than a thousandth of the conduction; heat flow by convection is less than that by radiation. Based on manufacturer's specifications and empirical data and for an ambient temperature of $23^{\circ} \mathrm{C}$ the junction temperature was taken as $\mathrm{T}_{\mathrm{J}}=23+0.314 \mathrm{P}$ The temperature under the chip is $\mathrm{T}_{2}=\mathrm{T}_{J}-0.012 \mathrm{P}$, and the case temperature is $\mathrm{T}_{\mathrm{C}}=23+\mathrm{P}^{0.7}$, where the power dissipation, $\mathrm{P}$, is in milliwatts.

* In an earlier analysis [14] of lead wire flexing due to heating, the expansion due to self heating by high current pulses was primarily considered although chip heating was involved. 
Thermal expansion was assumed to be linear over the test range and the expansion coefficients were taken as 2,5 , and $24 \times 10^{-6} /{ }^{\circ} \mathrm{C}$ for silicon, iron-nickel-cobalt header, and aluminum, respectively. The header expansion was assumed to be due only to the change in case temperature; local heating under the die only increases internal header stress. Chip expansion was calculated on the basis of a uniform temperature taken to be the arithmetical average of the top and bottom temperatures. The change in length of the various elements is then:

$$
X(P)=X_{0}[1+\alpha(T-23)],
$$

where $\mathrm{X}_{0}$ is the length of the element at ambient temperature, $\alpha$ is the thermal expansion coefficient of the material, and $\mathrm{T}$ is the temperature of the element, a function of the power dissipation, P. The wire is attached at points on the post and the chip. The bond-to-bond distance, L, is given by:

$$
L=\left[d^{2}+(Y-Z)^{2}\right]^{1 / 2},
$$

where $d$ is the bond-to-bond distance in the plane of the header, $Y$ is the post height and $Z$ is the die height.

Calculations were made for two cases, a circular arc and a triangular wire loop; bending moments in the wire were neglected. The equation for the wire length, $S$, in the circular arc model is

$$
\mathrm{S}=\mathrm{S}_{0}\left[1+\alpha_{\mathrm{Al}}\left(\frac{2 \mathrm{~T}_{\mathrm{J}}+\mathrm{T}_{\mathrm{C}}}{3}-23\right)\right]
$$

where $S_{O}$ is the length of the wire at $23^{\circ} \mathrm{C}, \alpha_{A l}$ is the thermal expansion coefficient for aluminum, $\mathrm{T}_{J}$ is the junction temperature, and $\mathrm{T}_{C}$ is the case temperature. The equations for the length of the die half, a, and the post half, $b$, of the wire in the triangular model is

$$
a=\frac{S_{0}}{2}\left[1+\alpha_{A 1}\left(\frac{5 T_{J}+T_{C}}{6}-23\right)\right]
$$

and

$$
\mathrm{b}=\frac{\mathrm{S}_{0}}{2}\left[1+a_{\mathrm{Al}}\left(\frac{\mathrm{T}_{J}+\mathrm{T}_{\mathrm{C}}}{2}-23\right)\right]
$$


The equation for post height, Y:

$$
Y=Y_{0}\left[1+\alpha_{K}\left(\frac{19 T_{C}+T_{J}}{20}-23\right)\right]
$$

where $\mathrm{Y}_{O}$ is the post height at $23^{\circ} \mathrm{C}$ and $\alpha_{K}$ is the thermal expansion coefficient of the post alloy, gives a post expansion less than 0.01 percent larger than that obtained previously with the assumption that the post is at the case temperature.

[717] The flexure can be defined as the change in loop height:

$$
\Delta \mathrm{H}_{\mathrm{L}}=\mathrm{H}_{\mathrm{L}}-\mathrm{H}_{\mathrm{LO}} \text {. }
$$

The functional relationship between loop height and the lengths $S$ and $L$ depends on the shape of the wire loop. If the wire loop is in the form of an arc of a circle, the loop height is given approximately by:

$$
H_{L}=[3(3 S+5 L)(S-L)]^{1 / 2 / 8} \text {, }
$$

If the loop is assumed to retain its circular shape during flexing, this expression is appropriate for calculations before and after flexing. The calculation is made by selecting a value for $\mathrm{H}_{\mathrm{LO}}$ and computing $\mathrm{S}_{\mathrm{O}}$ to obtain the initial condition. Then $\mathrm{S}, \mathrm{L}$, and $\Delta \mathrm{H}_{\mathrm{L}}$ are computed for various values of $\mathrm{P}$ to obtain the flexure as a function of power dissipation.

If the wire loop is in the form of a triangle, the loop height is given by:

$$
H_{L}=2[s(s-a)(s-b)(s-L)]^{1 / 2 / L} \text {, }
$$

where $a+b=S$ and $s=(a+b+L) / 2$. This formulation allows the difference in expansion of the die and post ends of the wire, which results from the temperature distribution along the wire, to be taken into account.

The curves of flexure (change in loop height, $\Delta \mathrm{H}_{\mathrm{L}}$ ) plotted against power input to the junction, $\mathrm{P}_{\mathrm{D}}$, (or, equivalently, junction temperature, $\mathrm{T}_{\mathrm{J}}$ ) are shown in Fig. 4-2 for initial loop heights, $\mathrm{H}_{\mathrm{LO}}$, of $50,100,250$, and $500 \mu \mathrm{m}$ for the circular arc model (dashed lines) and the triangular model (solid lines).

From these plots, it can be seen that regardless of the details of the model the flexure is approximately proportional to power dissipated and approximately inversely proportional to initial loop height.

(W. E. Phillips) 


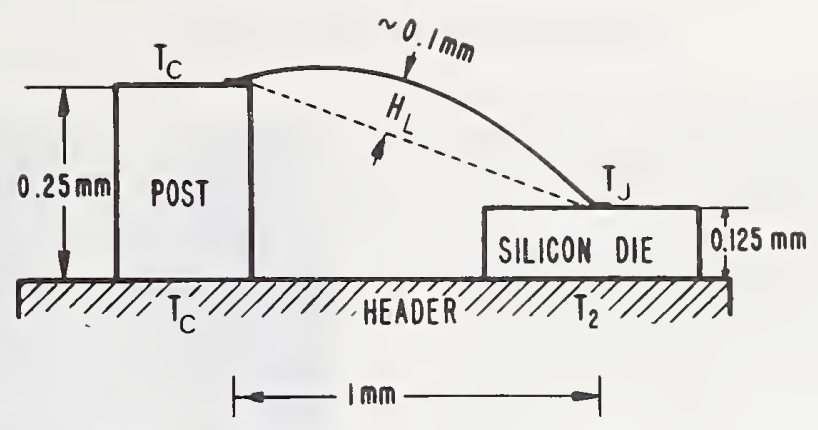

Fig. 4-1. Schematic representation of device structure used in calculation of wire-bond flexure.

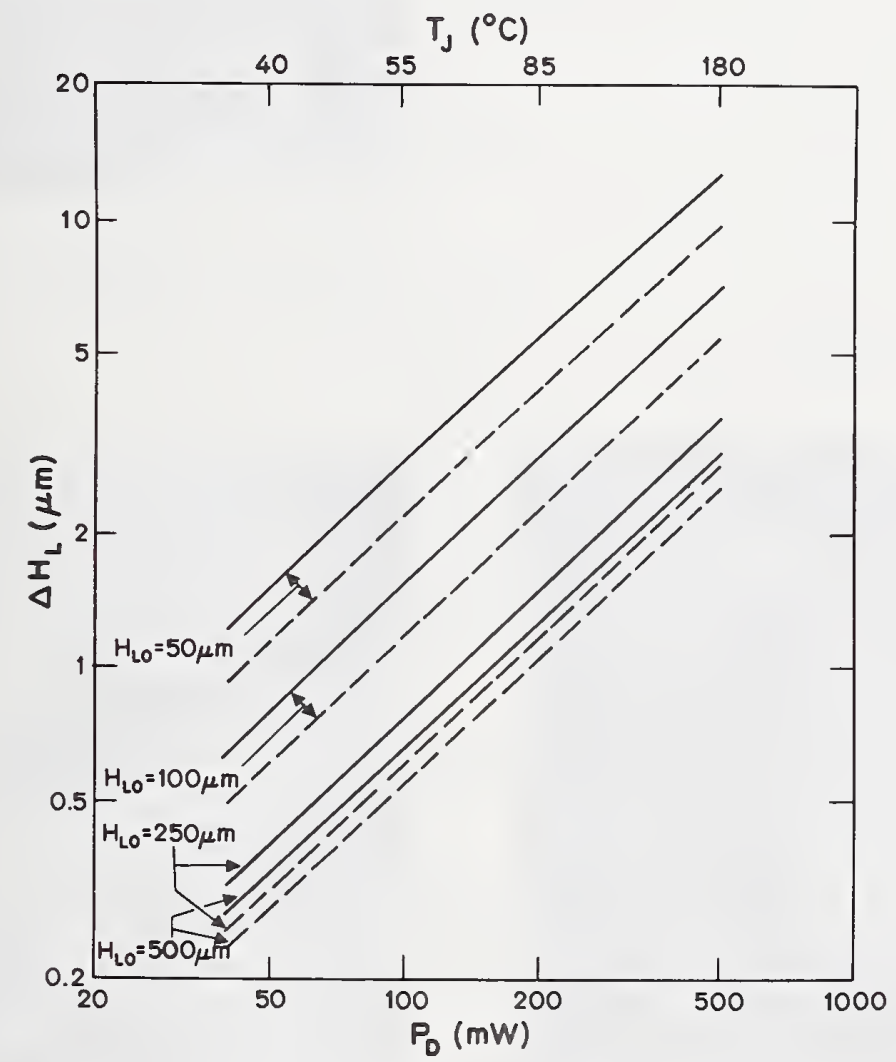

Fig. 4-2. Wire-bond flexure, $\Delta \mathrm{H}_{\mathrm{L}}$, as a function of power dissipation, $\mathrm{P}_{\mathrm{D}}$, for triangular (solid lines) and circular arc (dashed lines) bond loops with various values of initial loop height, $\mathrm{H}_{\mathrm{LO}}$. (The analysis was made for 1 mil diameter post-to-die aluminum wire bonds in a 500-mW, $50-\mathrm{mA}$ silicon transistor in a T0-18 can with post and header of the usual iron-nickelcobalt alloy. The junction temperature, $\mathrm{T}_{\mathrm{J}}$, is also indicated.) 
Fig. 4-3. SEM photomicrographs of ultrasonic aluminum wire bonds made with large loop helghts showing variation in bond deformation. At the time of the SEM observations the transistors had endured 76,000 power cycles. The hillock growth on the aluminum metallization is a result of the power cycling.

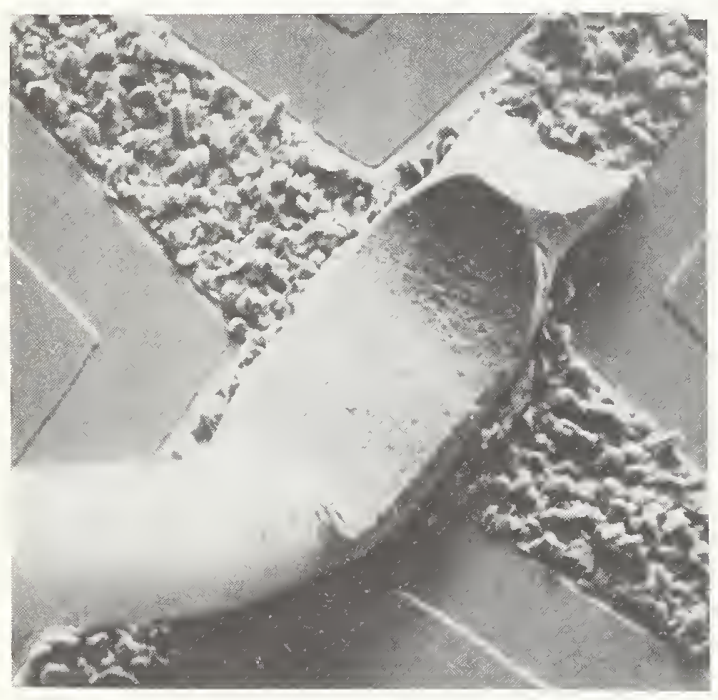

b. Average bond deformation 21.6 times wire diameter as determined from head-on SEM photomicrograph. Magnification $2460 \mathrm{x}$.

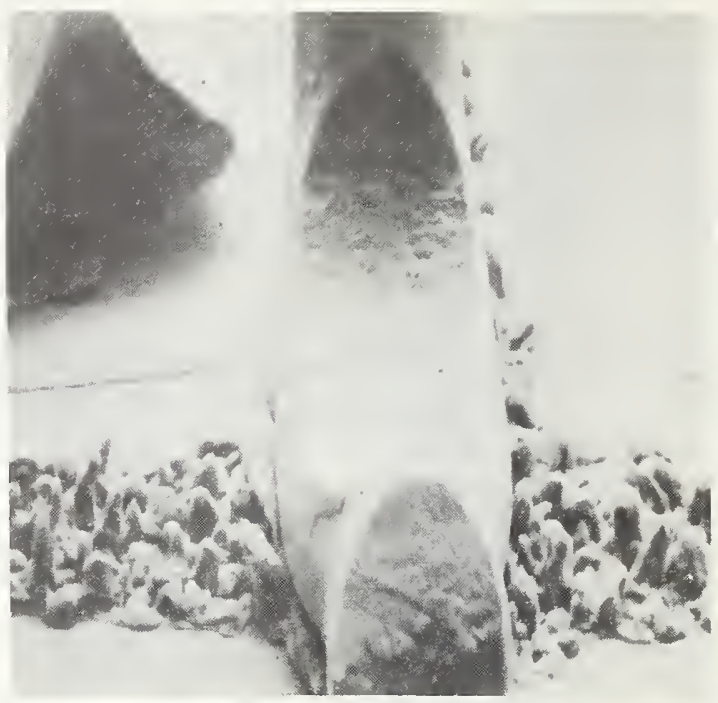

a. Small bond deformation 21.2 times wire diameter. Nagnification $2585 \times$.

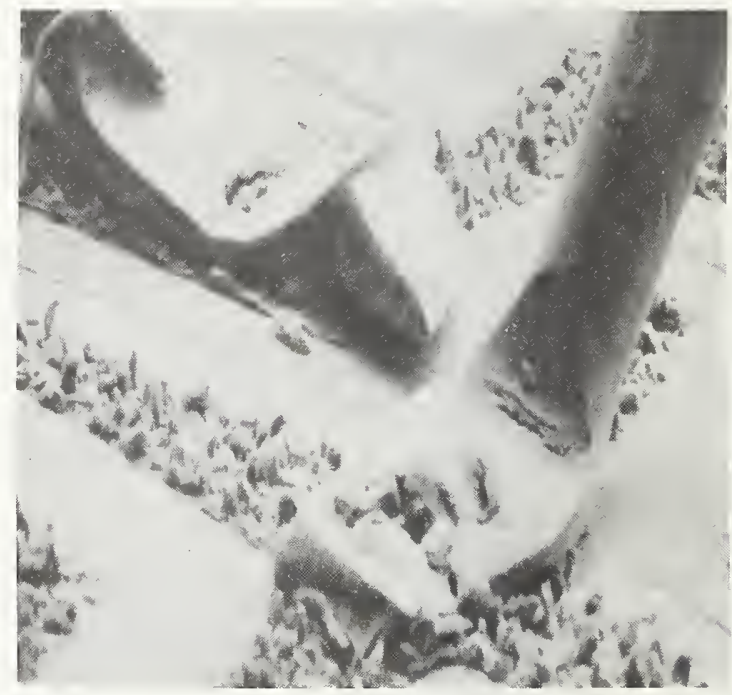

c. Large bond deformation $\sim 2.2$ times wire diameter as determined from head-on SEM photomicrograph. Magnification $2230 \mathrm{x}$. 
The results of calculations (4.1.1) have shown that an increased loop height would be expected to minimize wire bond flexure during slow power cycling, and increase the life of the devices. Observations were carried out with a scanning electron microscope (SEM) on a sampling of 2 N2222 transistors that had been fabricated with one mil diameter aluminum, $1 \%$ silicon wire, having loop heights in the range 0.012 to $0.015 \mathrm{in}$. (300 to $375 \mu \mathrm{m})$, significantly higher than normal, and then subjected to cycling at full rated power for 2 minutes on and 2 minutes off* at the NASA Marshall Space Flight Center. Five devices, part of a lot of 100 transistors that had been cycled 76,000 times with no failures, were examined.

There was considerable variability in the deformation of the wire bonds to the semiconductor chip. Based on the observation of 10 such bonds, it appeared that bond deterioration, such as heel cracking and loss of material in the center of the bond, was pronounced when the bond deformation was greater than about two wire diameters. Typical examples are shown in the SEM photomicrographs in Fig. 4-3. Despite the severe bond deterioration observed in some cases, the remaining 95 devices of the original group have withstood 30,000 additional power cycles without failure. The long life of these devices confirms that the high loop heights reduced wire flexure fatigue, compared to earlier devices fabricated with the same wire but having loop heights of only 4 to $5 \mathrm{mils}(0.1$ to $0.13 \mathrm{~mm})$. In such devices failures began to occur from a few hundred to a few thousand of the 2 minutes on and 2 minutes off power cycles. In addition to producing great resistance to power-cycling-induced-failure, such high loops also result in high bond pull strengths, as shown in section 4.2 .

(W. E. Phillips, K. O. Leedy, and G. G. Harman)

\subsubsection{Conclusions: Loop Height Requirements}

The conclusion of this study is that for one mil diameter $1 \%$ silicon, aluminum wire in geometries similar to that of Fig. 4-1 a loop height of 10 to 12 mils $(0.25$ to $0.3 \mathrm{~mm})$ should give the optimum practical protection against power or thermal-cycle-induced wire-flexure-fatigue.

* This is the minimum cycle time required to establish thermal equilibrium in these devices on each half of the cycle. Decreasing the cycle time, say to one minute on and one off, would considerably reduce the change in temperature $(\Delta \mathrm{T})$ and thus the amount of wire flexing. This would extend the wire-bond life to many times that of the longer cycle. Later tests carried out on $1 \%$ magnesium, aluminum wire under the same program (Circuits Manufacturing, p. 22-30, Jan. 1973) used the shorter cycle where $\Delta \mathrm{T}$ may only be 30 to $50 \%$ of the $\Delta \mathrm{T}$ experienced by the high loop devices. 
This conclusion should be valid for other geometries as long as the ratio of loop height (as defined in Fig. 4-1) to bond length is $\gtrsim 25 \%$. It will not hold for large wire sizes where the stiffness of the wire becomes a significant factor in the flexibility of the loop, for instance aluminum wire having a diameter $\approx 5 \mathrm{mils}(0.13 \mathrm{~mm})$. However, ribbon wire (see NBS Technical Note 767 ) of large cross section area may be advantageous in this case.

An alternative solution to using $1 \%$ silicon, aluminum wire with large loop heights is to use gold bonding wire with moderate loop heights. The basis for the above flexure calculations was the differential coefficient of expansion between the wire and the iron-nickel-cobalt alloy header. This differential is significantly smaller for gold than for aluminum wire. The use of gold wire with the same loop height as aluminum reduces the wire flexing in the device by about one half for the same power dissipation. In addition, gold has a higher metallurgical resistance to flexure fatigue. To realize the advantages of gold wire, consideration must be given to the possible formation of intermetallic compounds if the gold wire is attached directly to aluminum films and is subjected to a high temperature during testing or operation. Another alternative may be the use of $1 \%$ magnesium, aluminum wire which has recently been suggested by Ravi and Philofsky [17] as a metallurgical improvement over the usual $1 \%$ silicon, aluminum wire to reduce flexure fatigue.* (W. E. Phillips)

\subsection{The Effect of Loop Height on Bond Pull Strength}

In Section 4.1 it was shown that a large loop height increases wire bond reliability during power (thermal) cycling. A large loop height also has the advantage that it increases the bond pull strength. Fig. 4-4 is a calculated pull strength vs loop height curve based on equations published in NBS Technical Note $726, p .60$. The curve is calculated specifically for one value of bond to bond distance $(0.04 \mathrm{in.}, 1 \mathrm{~mm})$ and four post heights, rather than attempt to give general curves in terms of various parameters. Everything else being equal the general conclusion, large loops yield large pull strengths, is valid for any device geometry. The results of this type of resolution of forces calculation have been experimentally verified at NBS (see NBS Tech. Note 788, pp. 30-35). The major discrepancy between calculation and experiment is that for very large loop heights ( $~ 0.016$ in., $0.4 \mathrm{~mm}$ ) some bonds apparentiy peel off rather than break at the bond heel. This may lead to a more rapid leveling off of pull strength than is indicated in the calculated curve.

(G. G. Harman)

* See footnote p. 85 


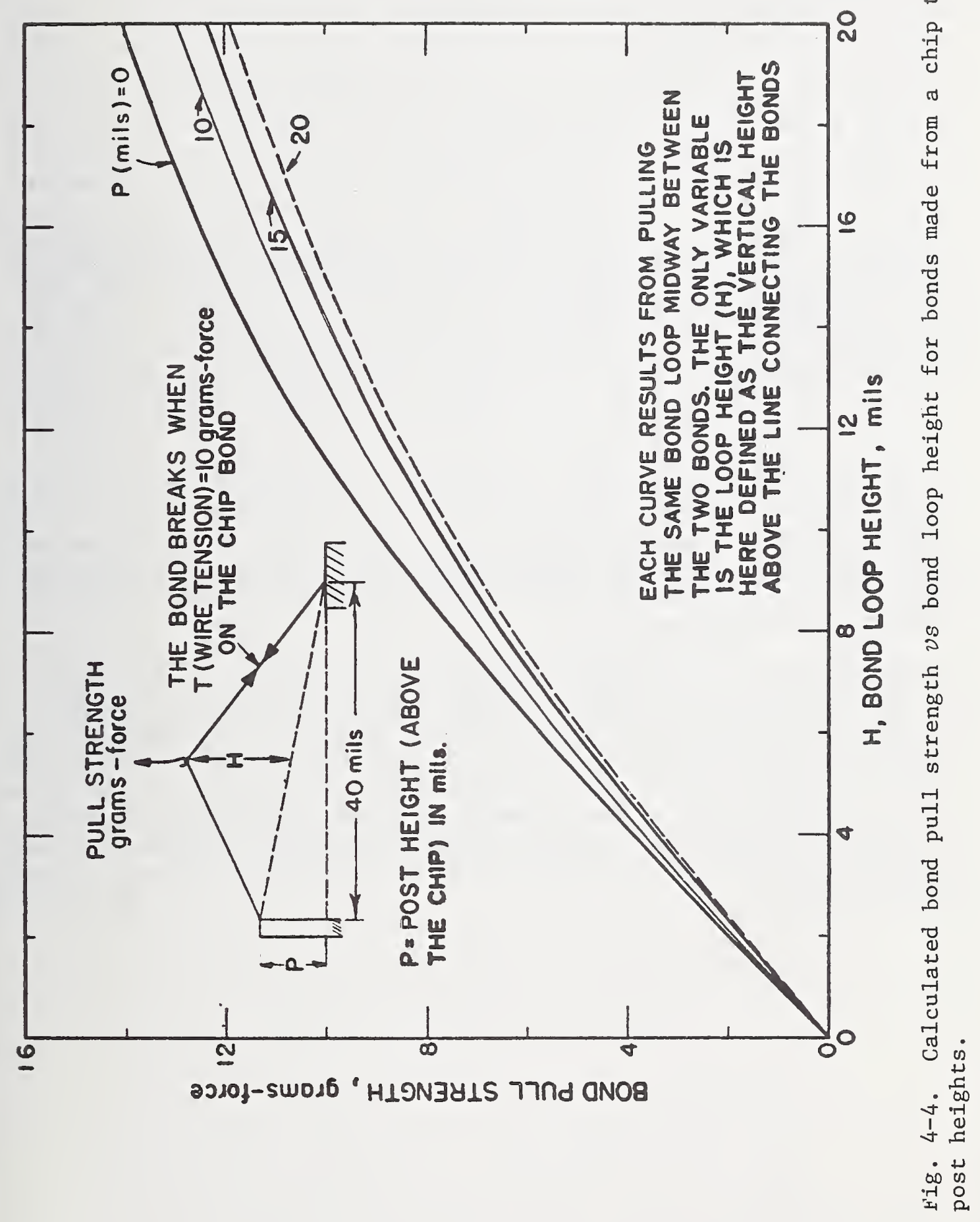


[598] Most manufacturing wire-bond acceptance criteria do not take into account the possibility of later bond degradation that may result from the operating environment of the finished device. Many devices or systems are turned on and off periodically during their life and therefore undergo heating and cooling cycles. This causes thermal-expansion-induced wire flexing that can eventually result in bond failure (Section 4.1). Available data [15] indicate that failures due to temperature-cycling occur principally at the bond on the semiconductor chip. This suggests that it is desirable to have a bond pull-strength criterion that is dependent upon the quality of the metallurgical weld, the bond deformation, and any strain or cracking in the bond heel. One possible criterion, termed bond efficiency, that may satisfy the above requirements is based upon the ratio (expressed as percent) of the actual force in the wire $\mathrm{T}_{\mathrm{b}}$ when the bond loop breaks, to the breaking strength of the wire, $\mathrm{T}_{\mathrm{W}}$. Such a criterion has been used in the resistance welding field for many years with acceptable bond efficiencies generally ranging from about 50 to $90 \%$, depending on circumstances. There have been several recent references that apply this bond efficiency concept to ultrasonic wire bonding $[18,19]$. Bond efficiencies of from 80 to $90 \%$ have been achieved using wires of very different tensile strengths, when proper bonding machine parameters were chosen [19]. Similar results have been obtained in the present NBS wire bonding program. Also bonds made on one high-reliability production line show bond efficiencies consistently greater than $60 \%$, and there have been no wire-bonding field-failures reported in the several years of that program.

As an example the use of bond efficiencies, consider a typical 1 mil (25 $\mu \mathrm{m}$ diameter aluminum wire with a breaking strength of $13 \mathrm{gf}(128 \mathrm{mN})$. If the minimum acceptable value of $\mathrm{T}_{\mathrm{b}} / \mathrm{T}_{\mathrm{W}}$ be chosen as $60 \%$, a bond to be acceptable must break with a force not less than $7.8 \mathrm{gf}(76 \mathrm{mN})$ in the wire. To translate this into the measured pull strength, it is necessary to consider the bond to bond geometry. Assuming the geometry of Fig. 4-1 and that the bond loop is pulled vertically, halfway between the bonds, a pull strength of $3.3 \mathrm{gf}(32 \mathrm{mN})$ would be obtained. Additional values are given in Table $I$.

(G. G. Harman) 
Table I

Calculated pull strengths in grams force ( $\mathrm{gf}$ ) for the three values of bond efficiency $\mathrm{T}_{\mathrm{b}} / \mathrm{T}_{\mathrm{W}}$ and two values for the wire breaking strength, $\mathrm{T}_{\mathrm{W}}$. The pull strengths are calculated by the resolution of forces for a wire bond of a typical 2N2222 transistor mounted in a TO-18 header (see Fig. 4-1). The loop height used is 4 mils. For an $8 \mathrm{mil}$ loop multiply the pull strength by 2 (see Fig. 4-4).

\begin{tabular}{|c|cc|}
\multirow{2}{*}{$\begin{array}{c}\text { Bond Efficiency } \\
\mathrm{T}_{\mathrm{b}} / \mathrm{T}_{\mathrm{W}}(\%)\end{array}$} & \multicolumn{2}{|c|}{ Pull Strength (gf) } \\
\cline { 2 - 3 } & $\mathrm{T}_{\mathrm{w}}=13 \mathrm{gf}$ & $\mathrm{T}_{\mathrm{W}}=18 \mathrm{gf}$ \\
\hline 30 & $\sim 1.6$ & $\sim 2.2$ \\
\hline 50 & $\sim 2.7$ & $\sim 3.8$ \\
\hline 70 & $\sim 3.8$ & $\sim 5.4$ \\
\hline
\end{tabular}




\subsection{The Effect of Wire Elongation on Bond Pul1 Strength}

There are many situations where annealed bonding wire is used in semiconductor devices. Power devices may use large, 5- to 20-mil (0.13- to $0.5-\mathrm{mm}$ ) diameter, annealed wire having elongations of up to $20 \%$. Microelectronic devices are often made in ceramic packages that are subsequently glass sealed at approximately $500^{\circ} \mathrm{C}$. The breaking strength of the resulting annealed wire may be less than one-third of its precap value, and the elongation typically increases to 8 or $10 \%$. The bond pull strength is generally expected to be directly proportional to the reduced breaking strength of the wire. However, the bond pull strength in typical device geometries [4- to 5-mil (0.1- to 0.13-mm) loop heights and 40 - to 50-mil bond lengths] is seldom degraded more than $25 \%$ by annealing. The reason for this is revealed by examining the effect on breaking strength of bond loop height as modified by wire elongation.

Section 4.2 showed that loop height is an important factor in determining the bond pull strength. Thus it is apparent that significant wire elongation during bond pulling will change the loop height and affect the magnitude of the pull strength. Fig. 4-5 gives the loop height change vs elongation for three different bond to bond lengths, all starting with the same initial loop height. The geometries were chosen to cover those often encountered in medium- to high-power transistors but they can be linearly scaled down to appropriate microelectronic dimensions as long as the ratio of loop height to bond spacing is kept constant.

Fig. 4-6 shows the effect of this wire elongation (incorporating the resulting loop height increase) on the bond pull strength, assuming the same initial geometry as in Fig. 4-5. In this calculation, all bonds break when the force in the wire reaches 500 grams, a reasonable value for 10-mil diameter wire bonds. For simplicity, the calculation was made for single level bonds.

In practice, for different wire annealing conditions the wire breaking strength and the elongation are inversely proportional, as indicated by the shaded area of Fig. 4-6. Specific data for this area was obtained from stress relieved and annealed, 10-mil diameter, aluminum wire alloys containing $1 \%$ silicon, or $0.5 \%$ and $1 \%$ magnesium, as well as alloy 1100 . Wire from different manufacturers or even different lots from the same manufacturer will vary somewhat but the general shape of the curve will 


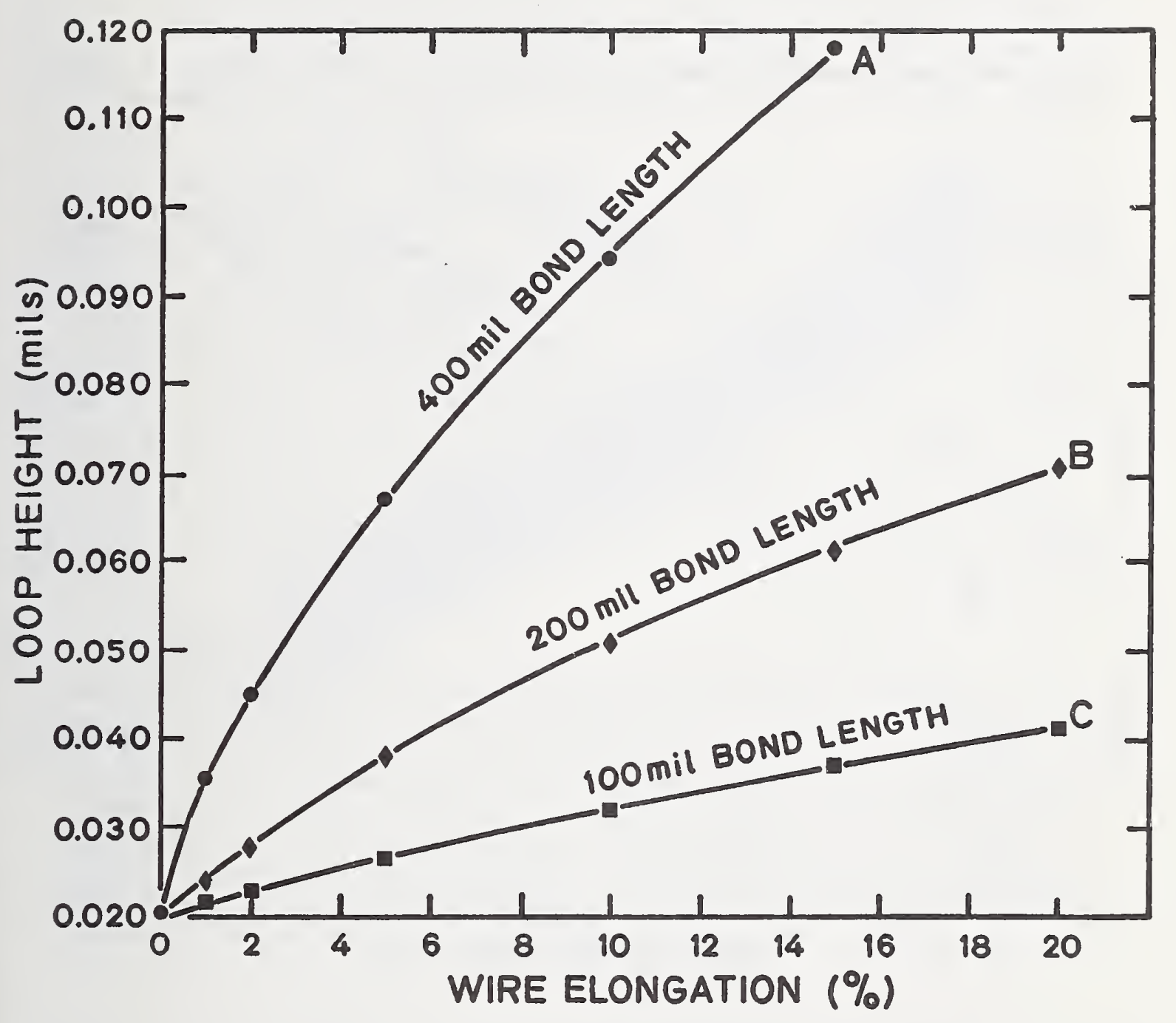

Fig. 4-5. The effect of wire elongation on the final loop height at the point of wire rupture during a bond pull test. All bonds had the same initial loop height, $20 \mathrm{mils}(0.5 \mathrm{~mm})$ but three bond to bond lengths were used. 


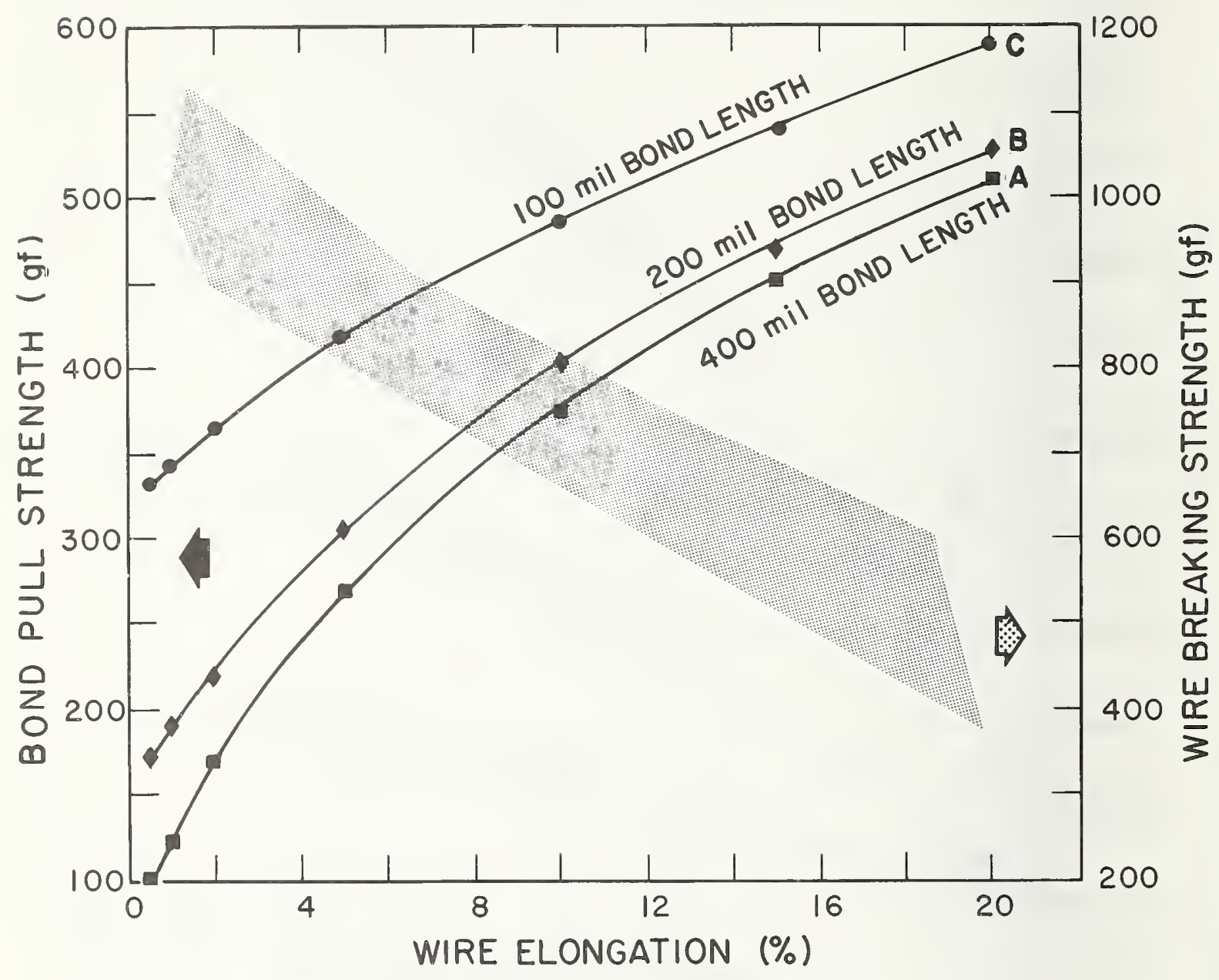

Fig. 4-6. Bond Pull Strength and Wire Breaking Strength vs Wire Elongation. The solid curves are bond pull strengths based on the geometries of similar lettered curves in Fig. 4-5. The shaded area represents the breaking strengths of four typical $10 \mathrm{mil}$ diameter aluminum wire alloys in the stress relieved and the annealed condition. As the breaking strength decreases due to annealing, the elongation increases. 
be similar.* From Fig. 4-6 it is apparent that the tendency of the bond pull strength to decrease with decreasing wire breaking strength will be partially or entirely offset by the increase in bond pull strength resulting from increased wire elongation. Therefore, the pull strengths tend to be independent of the wire breaking strength for many common device geometries. These results may be scaled down for integrated circuits, except that small diameter annealed wire does not elongate more than approximately $10 \%$.

There will be cases where the effect of large wire elongations can significantly change the bond-pull geometry and hence the measured pull strength, if the pulling probe (the hook and arm) is flexible or is free to pivot where it is joined with the force gauge. This may occur in the case where a large post height is involved. Here the wire span will be considerably longer on the chip side of the hook than on the post side. The relatively greater increase in length of the chip-side span during the test will result in moving the hook nearer the post and in pulling on the wire at some angle from the vertical. The effect will be enhanced if the pulling hook were initially placed nearer to the post than to the chip bond. These changes in the bond pull geometry, which can result in lower values of pull strength, must be taken into account in any pull test calculations.

(G. G. Harman)

* Hard drawn wire (seldom used for large wire bonding) may have a breaking $10 a d 30 \%$ higher, but an elongation only slightly lower than that of stress relieved wire. Use of the hard drawn wire could therefore result in a $30 \%$ higher bond pull strength than that obtained from the stress relieved or annealed wire. Aluminum wire of $99.999 \%$ purity (again seldom used) has a breaking strength about $30 \%$ lower than the alloys shown in Fig. 4-6; however, the elongation may be as high as $30 \%$. Due to the complex metallurgy involved, occasionally wires from any manufacturer will fall well outside the shaded area of Fig. 4-6. 


\subsection{UTtrasonic Bondability of Gold Wire}

[806] A series of experiments was run to determine the ultrasonic bondability of gold wire and some of its alloys. Gold wire of 99.99 percent purity, from a single manufacturer was used. The wire had a diameter of $1 \mathrm{mil}$ $(0.025 \mathrm{~mm})$, a tensile strength of $13 \mathrm{gf}(0.127 \mathrm{~N})$, and an elongation less than 2 percent. All bonds were made on $0.9-\mu \mathrm{m}$ thick aluminum bonding pads evaporated on a film of silicon dioxide, $0.8 \mu \mathrm{m}$ thick, thermally grown on a silicon slice. A formal statistical set-up procedure (section 2.1) was carried out to establish the appropriate bonding schedule. Bonding forces of 23,30 , and $36 \mathrm{gf}(0.23,0.29$, and $0.35 \mathrm{~N})$; bonding times of $45,70,105,135$, and $160 \mathrm{~ms}$; and eight ultrasonic tool tip vibration amplitudes in the range 20 to $85 \mu$ in. $(0.51$ to $2.2 \mu \mathrm{m})$ peak-topeak were employed. These variations were carried out separately on both the first and the second bonds. Ten to fifteen single-level bonds, with bond-to-bond spacings of 38 to $40 \mathrm{mils}(0.96$ to $1.02 \mathrm{~mm})$ and $100 \mathrm{p}$ heights of 11 to $12 \mathrm{mils}(0.28$ to 0.30$)$, were made in each group to obtain one datum. The optimum force and time were found to be $30 \mathrm{gf}(0.29 \mathrm{~N})$ and $45 \mathrm{~ms}$, respectively. Measured pull strengths of bonds made with these optimum bonding schedule parameters are given in Fig.4-7a as a function of tool tip vibration amplitude. All bond loops broke or lifted off at the first bond. Also shown in the figure are the average wire deformation in wire diameters (upper horizontal axis) and power supply dial setting (small numbers by each datum). The error bars represent one sample standard deviation above and below the mean.

From resolution of forces (NBS Tech. Note 726, p. 60) the indicated pull strength is also the actual force in the wire at rupture. The bond efficiency, the ratio of pull strength to wire tensile strength, was greater than 65 percent, an unexpectedly high value, over a very wide range of ultrasonic power. Even at very large bond deformations ( 3 wire diameters) the average pull strength of the gold wire was greater than $7 \mathrm{gf}(0.069 \mathrm{~N})$ This appears to be possible because of the metallurgical properties of gold. Aluminum is reported to work harden during ultrasonic welding [20] resulting in a damaged, brittle bond heel. An aluminum wire bond, having the same deformation, geometry, and wire breaking strength as the gold wire has an average pull strength in the 3 to $4 \mathrm{gf}(0.029$ to $0.039 \mathrm{~N})$ range. A comparison bonding machine setup for aluminum wire made with the same bonding machine used for the gold wire is given in Fig. 4-7b. The thinned, widened heel of a gold wire bond appears to retain the normal strength of its remaining cross sectional area because there is little ultrasonic work damage. Such strength at high deformation is well known in thermocompression wedge bonding, but in that case it has been generally assumed that the heat during bonding is sufficient to recrystallize the material as it is being deformed.

(C. A. Main and G. G. Harman) 


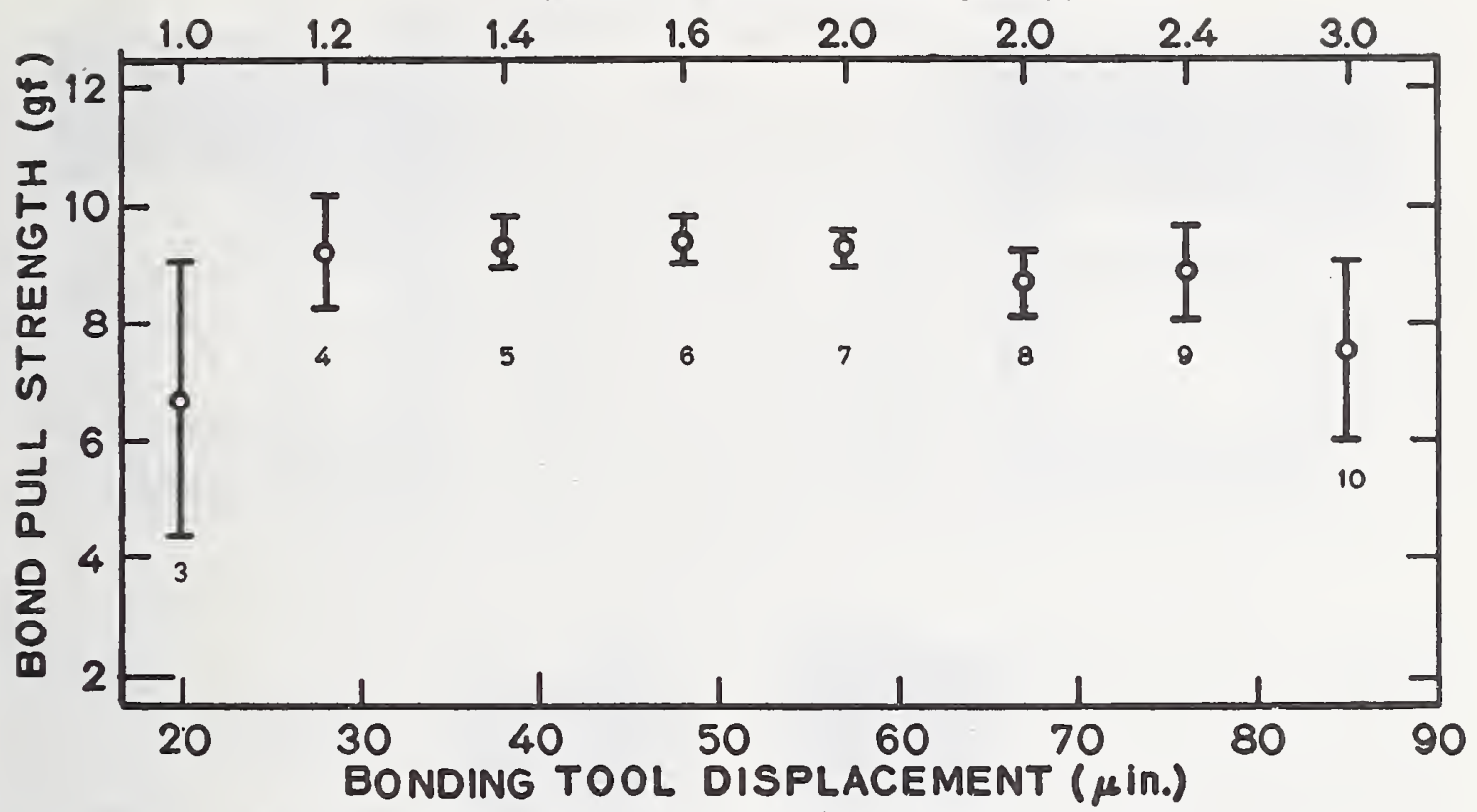

Fig. 4-7a. Bond pull strength vs bonding tool displacement amplitude (ultrasonic power) and bond deformation, for 1 mil diameter gold-wire bonded to thin-film $(0.8 \mathrm{\mu m})$ aluminum on silicon. The error bars represent \pm one standard deviation of the mean, and the small numbers under the error bars are the ultrasonic power supply power-dial settings. The bonding force was $30 \mathrm{gf}$. The bonding time was $45 \mathrm{~ms}$, and the bondingtool foot-length was $4.5 \mathrm{mils}(0.11 \mathrm{~mm})$.

\section{TYPICAL BOND DEFORMATION (wire diameters)}

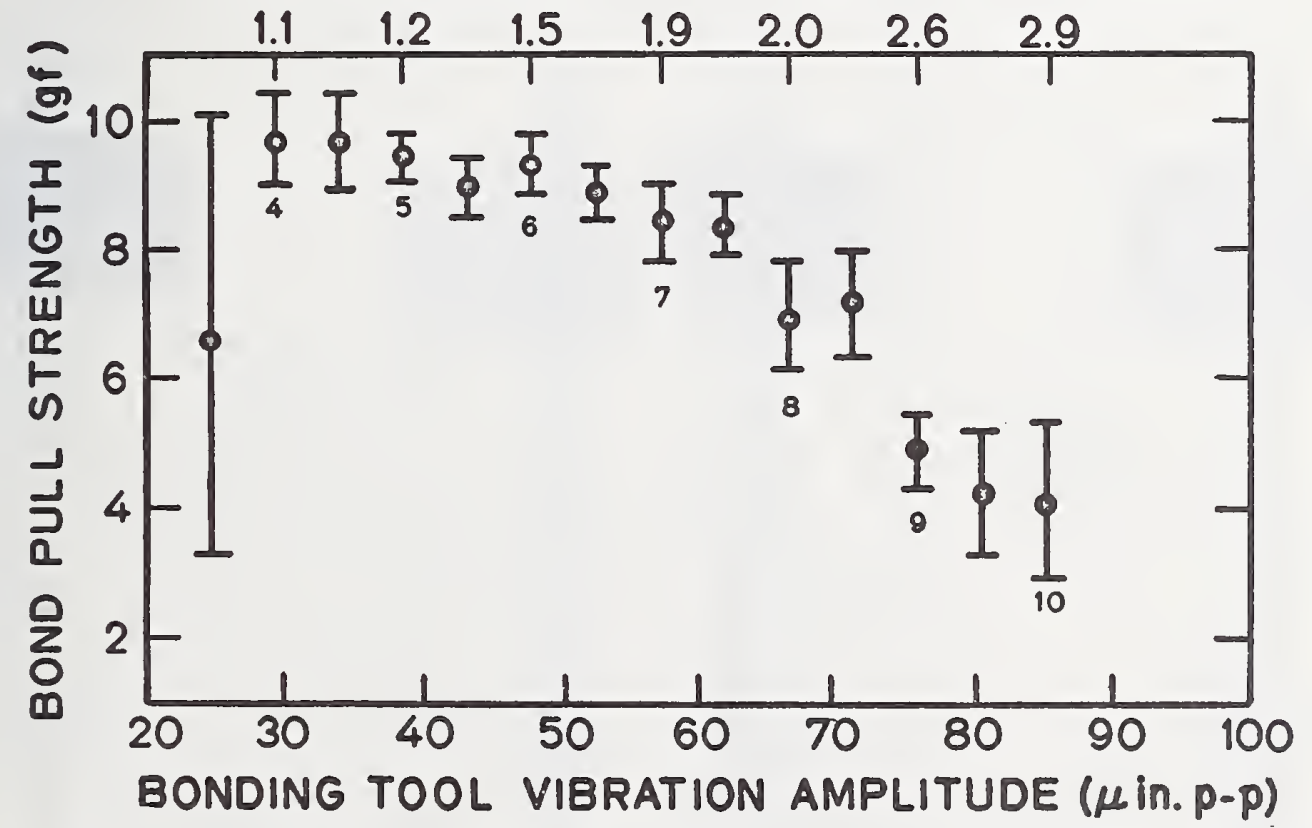

Fig. 4-7b. Bond pull strength vs bonding tool displacement amplitude for one mil diameter $1 \%$ silicon aluminum wire. The bonding parameters are the same as for (a) except that the bonding force was $25 \mathrm{gf}$. 

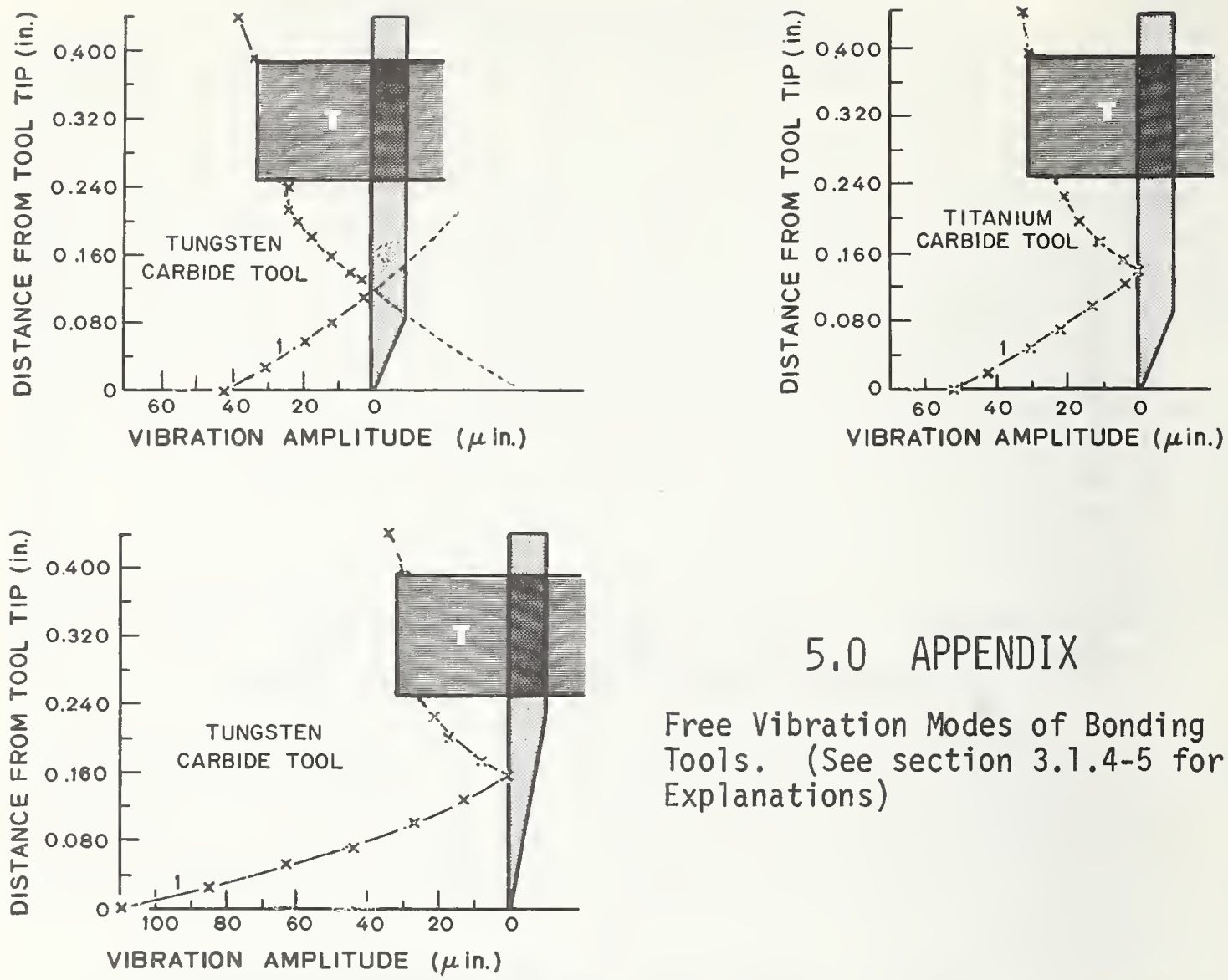

\subsection{APPENDIX}

Free Vibration Modes of Bonding Tools. (See section 3.1.4-5 for Explanations)
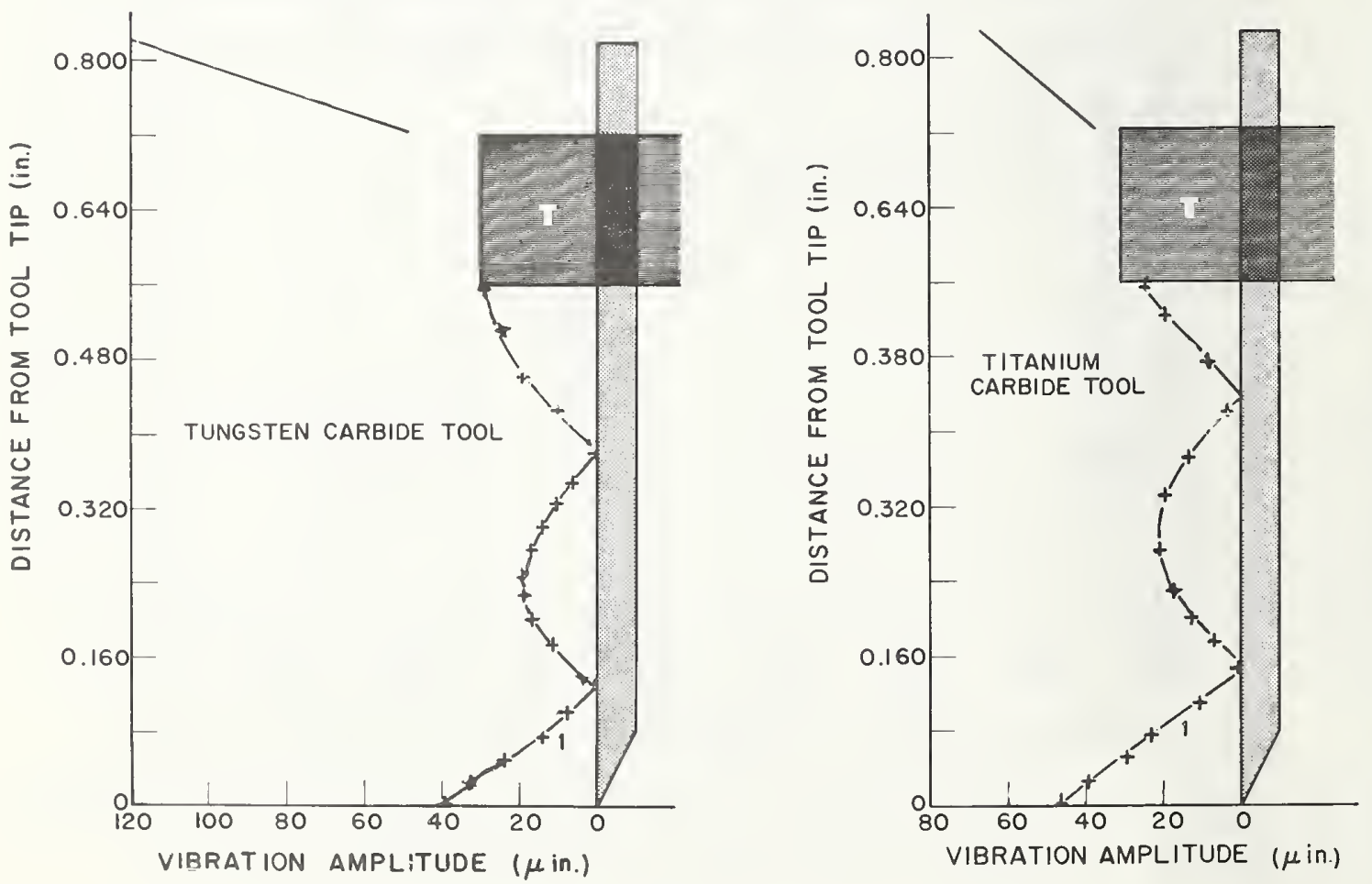

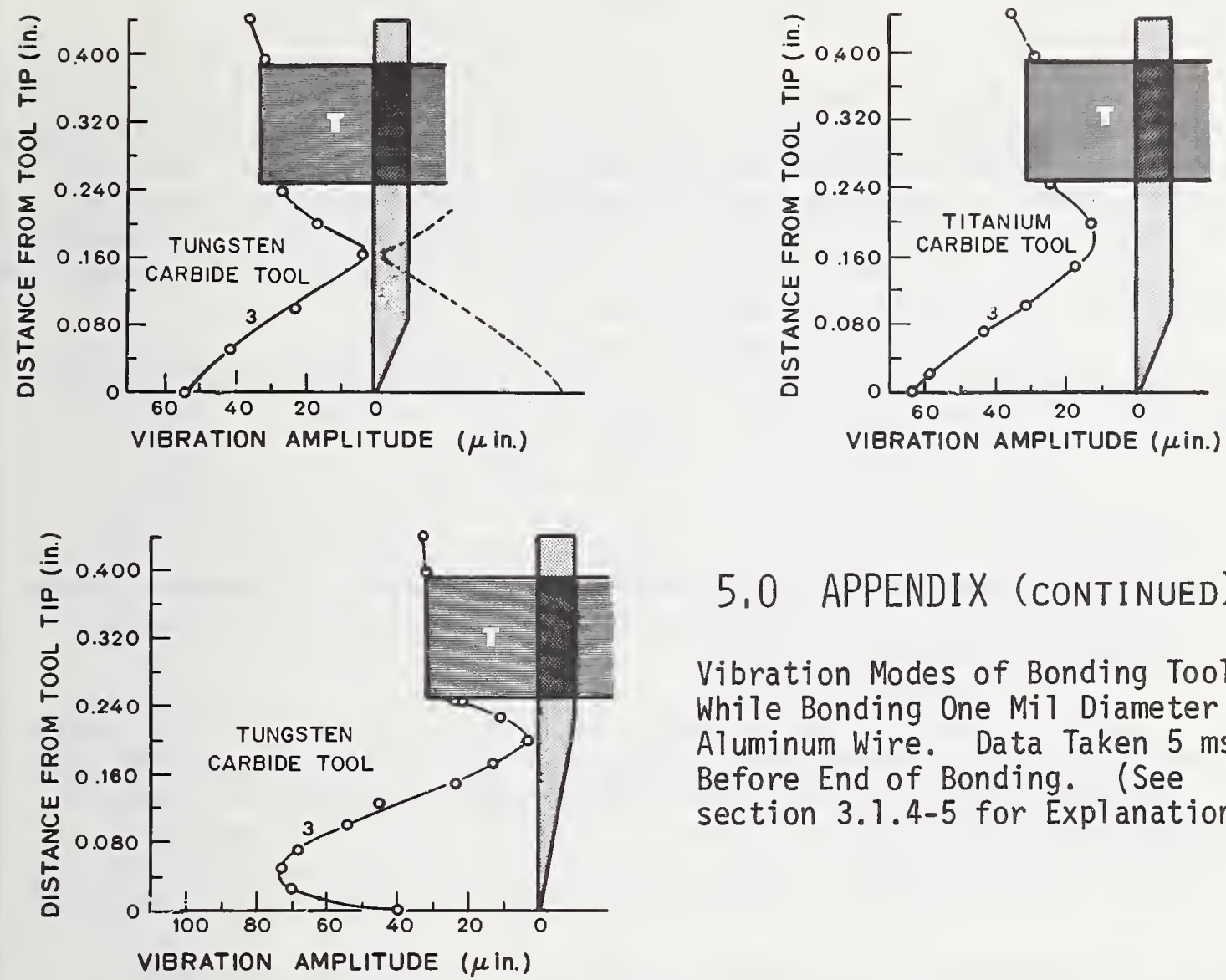

\subsection{APPENDIX (CONTINUED)}

Vibration Modes of Bonding Tools While Bonding One Mil Diameter Aluminum Wire. Data Taken $5 \mathrm{~ms}$ Before End of Bonding. (See section 3.1.4-5 for Explanations)
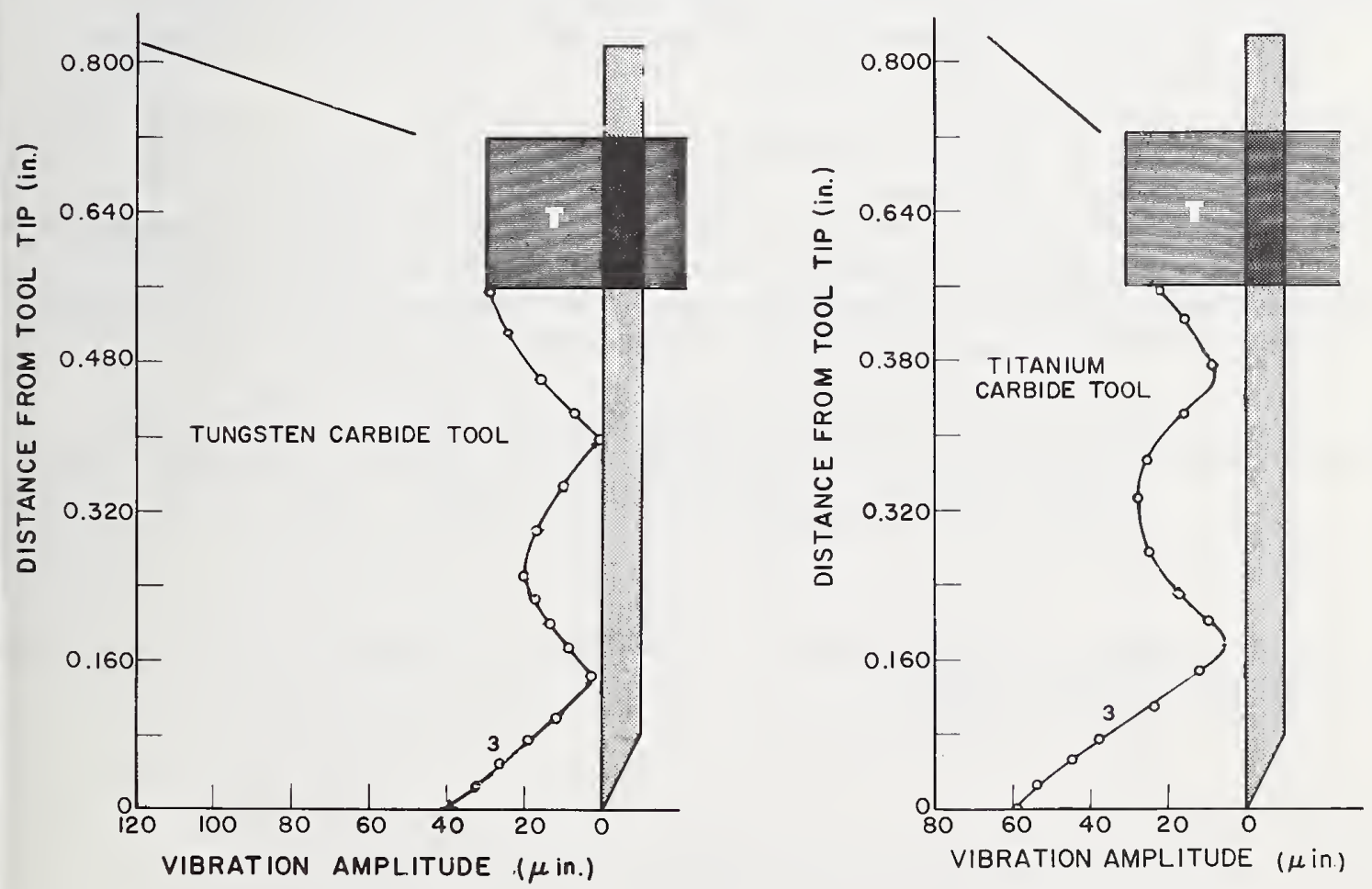


\subsection{REFERENCES}

1. The use of magnetic pickup to study variations in vibration amplitude of bonding tools set at different extensions was reported by D. Davis, Physics of Reliability Symposium, Las Vegas, Nevada, April 7-10, 1970. A more detailed discussion of this work was given in "Interim Progress Summary for January through June 1969" to Sandia Corporation, prepared by Fairchild Research \& Development Laboratories, Palo Alto, California. (Unpublished)

2. D. M. Clunie and N. H. Rock, Jr., The Laser Feedback Interferometer, J. Sci. Instrum. 41, 489-492 (1964).

3. B. D. Martin, presented at New York State Technical Services Symposium on Lasers and Their Application in Local Industries, Binghampton, N. Y., April 14, 1969, also B. D. Martin and A. D. Wilson, An In-Line Laser Interferometer Applied to U1trasonic Bonder Characterization, 1970 IEEE U1trasonics Symposium, San Francisco, California, October 21, 1970.

4. S. Bonis, "Poseidon Semiconductor Captive Line Process Development," Progress Report, 16 November 1968 to 15 March 1969, Report R69-4198, Raytheon Company, Space and Information Systems Division, Sudbury, Massachusetts 01776.

5. Such as Shell VPI \#250, Shel1 Oil Company, New York, New York 10020.

6. G. C. Knollman, A. S. Hamamoto, and J. L. S. Bellin, "Reports on U1trasonic Screening of Transistors and Integrated Circuits," LMSC B-62-29-8, Lockheed Palo-Alto Research Laboratory, Palo Alto, California 94304 (June, 1969).

7. P. M. Uthe, UTI Tech. Newsletter 1, No. 5 (August, 1968).

8. S. Bonis, "Poseidon Semiconductor Captive Line Process Development", Progress Report, 16 September 1968 to 15 November 1968, Report R68-4566, Raytheon Company, Space and Information Systems Division, Sudbury, Massachusetts 01776 (AD 844820L).

9. Neil Scott, Hughes Aircraft Company, Newport Beach, California (presently Centralab, E1 Monte, California) private communication.

10. T. Salzer, Raytheon Co., Sudbury, Massachusetts, private communication.

11. S. Z. Dushkes, A Design Study of Ultrasonic Bonding Tips, IBM J. Res. and Dev. 15230 (1971). 
12. G. G. Harman, U1trasonic Systems and Mechanisms of Bonding, presented at the Advanced Microelectronics Techniques Conference, Los Angeles, California, September 30, 1971.

13. G. G. Harman and K. O. Leedy, An Experimental Model of the Microelectronic Ultrasonic Wire Bonding Mechanism, Proceedings of the 1972 IEEE Reliability Physies Symposizm, Las Vegas, Nevada, April 5, 1972, pages 49-56.

14. J. Gaffney, Internal Lead Fatigue Through Expansion in Semiconductor Devices, IEEE Trans. Electron Devices ED-15, 617 (1962), also J. Gaffney, D. Bottaro and C. D. Root, Internal Lead Fatigue in Semiconductor Devices Through Thermal Expansion, presented at the Seventh Annual Reliability Physics Symposium, Washington, D. C., December 3, 1968.

15. F. Villella, and M. F. Nowakowski, Investigation of Fatigue Problem in One-Mil Diameter Thermocompression and Ultrasonic Bonding of Aluminum Wire, NASA Technical Memorandum TM-X-64566, November 30, 1970. Available from National Technical Information Service, Springfield, Va. 22151 (Accession No. N71-16494).

16. A. L. Edwards, TRUMP: A Computer Program for Transient and SteadyState Temperature Distributions in Multidimensional Systems, Lawrence Radiation Laboratory, University of California, Livermore, California, UCRL-14754, Rev. I, 1 May 1968. Available from the National Technical Information Service, Springfield, Va. 22151.

17. K. V. Ravi and E. M. Philofsky, Reliability Improvement of Wire Bonds subjected to Fatigue Stresses, Proceedings of the 1972 IEEE Reliability Physics Symposizm, pages 143-148.

18. Riben, A. R., Sherman, S. L., Land, W. V., and Geisler, R., Microbonds for Hybrid Microcircuits, Physics of Failure in Electronics vo1. 5, Eds. T. S. Shillday and J. Vaccaro, pp. 534-556 (Rome Air Development Center, 1967).

19. Cox, W. P., Anderson, E. E., and Anderson, J. H., Jr., Ultrasonic Aluminum Wire Bonding for Microelectronic Applications, Annals of Assurance Sciences $\underline{3}$, No. 1, 228-236 (1970).

20. Coucoulas, A., U1trasonic Welding of Aluminum Leads to Tantalum Thin Films, Trans. Met. Soc. of AIME 236, 587-589 (1966). 
Reports Used to Assemble the Present Special Publication

Quarterly Reports - NBS Joint Program on Methods of Measurement for Semiconductor Materials, Process Control, and Devices

Period Covered Tech. Note No. Page No. of Order No. Price Wire Bonding Section

\begin{tabular}{|c|c|c|c|}
\hline Oct.-Dec. 1968 & 475 & $20-22$ & C13.46:475 \\
\hline Jan.-March 1969 & 488 & $21-25$ & C13.46:488 \\
\hline April-June 1969 & 495 & $24-31$ & C13.46:495 \\
\hline July-Sept. 1969 & 520 & $32-45$ & C13.46:520 \\
\hline Oct.-Dec. 1969 & 527 & $31-46$ & $\mathrm{C} 13.46: 527$ \\
\hline Jan.-March 1970 & 555 & $27-36$ & C13.46:555 \\
\hline April-June 1970 & 560 & $29-38$ & C13.46:560 \\
\hline July-Sept. 1970 & 571 & $23-32$ & C13.46:571 \\
\hline Oct.-Dec. 1970 & 592 & $34-45$ & C13.46:592 \\
\hline Jan.-March 1971 & 598 & $20-28$ & $\mathrm{C} 13.46: 598$ \\
\hline April-June 1971 & 702 & $17-21$ & $\mathrm{C} 13.46: 702$ \\
\hline July-Sept. 1971 & 717 & $22-30$ & $\mathrm{C} 13.46: 717$ \\
\hline Oct.-Dec. 1971 & 727 & $29-45$ & C13.46:727 \\
\hline Jan.-March 1972 & 733 & $27-33$ & C13.46:733 \\
\hline Jan.-March 1973 & 788 & $30-43$ & $\mathrm{C} 13.46: 788$ \\
\hline April-June 1973 & 806 & $27-38$ & $C 13.46: 806$ \\
\hline
\end{tabular}

Special Reports on Bonding

$\begin{array}{llrr}\text { May } 1971 & 573 & \mathrm{C} 13.46: 573 & 35 \$ \\ \text { Jan. } 1972 & 593 & \mathrm{C} 13.46: 593 & 60 \$ \\ \text { Sept. } 1972 & 726 & \mathrm{C} 13.46: 726 & 125 \$ \\ \text { Apri1 } 1973 & 767 & \mathrm{C} 13.46: 767 & 50 \$ \\ \text { August } 1973 & 785 & \mathrm{C} 13.46: 785 & 55 \$\end{array}$

Available from Superintendent of Documents, U. S. Government Printing Office, Washington, D. C. 20402. 


\section{A}

Aluminum metallization (effect of sintering), 22

hillock growth, 84

Aluminum wire, 19, 90-95

Amplitude (ultrasonic bonding tools), 11-15, $65-73,94$

Audio monitoring (in-process bonding studies), 59

\section{B}

Bond deformation (effect of low frequency motion), $29-33$

with respect to ultrasonic power, 95

Bond efficiency, 88

Bond failure

due to bonding over a void, 70-72

due to device power cycling, 80-86

due to extraneous motion, 29-36

due to phase-locked loop power supply, $55-57$

Bond 1ift-off patterns, 73-79

Bond loop

effect on power cycling, 80-86

effect on pull strength, 86-93

Bond monitoring studies, 58-73

Bond pull strength, 18, 23, 25, $85-94$

statistical evaluation, 24

Bond puller (glue, hook), 16-18

Bond screen (nondestructive), 18

Bondability of sintered aluminum, 22 of gold wire, 94

Bonding force, 19, 95

Bonding machine, 15, 23-38

mechanical stability, $29-36$

setup procedures, 23-28, $94-95$

Bonding mechanism, 73-79

Bonding schedule, 23-26, 94-95

Bonding surface (sintering), 22

Bonding tool

composition, 65

design, 65

extension below transducer, 39, 45, 47

polishing techniques, 21

resonance condition, 45

set screw, 27,48

studies of vibration modes, $39,41,45$, $65-74,96$

vibration amplitude, $11-14,65-73,94$

after changing tool, $45-48$
Bonding wire

aluminum, 19, $90-95$

elongation, $90 \quad-92$

gold, $94-95$

large diameter, 90-93

Building vibration (effect on bonding), 29-33

\section{C}

Capacitor microphone, 4 conical tip adapter, 5

placement for in-process measurements, 60 procedure for using, 26

Clamp (wire, problems), 36

D

Daily check out procedure (bonding machine), 24

$E$

Efficiency (bond), 88

Efficiency (of transducer), 38

Elongation (wire), 90-92

Force (bonding), 19,95

G

Glue (hot-melt, bond puller),18

Gold wire, $94-95$

H

Harmonic content (in-process bonding studies), 61

Hot-melt-glue, bond puller, 18

I

In-process bonding studies, 58-73

L

Laser interferometer, 11-14, 65-66

Lift-off patterns (bond), 73-79

Loop, effect on power cycling, 80.85

Loop height (bond), $80-93$

M

Magnetic pickups, 9,58 procedure for using, 26 
Metallization (sintering), 22

Motion (extraneous) effect on band formation, 29-36

\section{N}

Non-destructive bond screen, 16

\section{P}

Phase-locked-1 oop ultrasonic power supply, 55

Phased burst, 61

Polishing technique (bonding tool), 21

Powder method of displaying vibration nodes, 14

Power cycling (effect of loop height), 80-86

Power supply (ultrasonic), 50-56

Problems in ultrasonic systems, 45-57

Pull strength (bond) $18,23,25,85-94$

Pull strength (statistical evaluation of), 24

R

Resonant frequency (bonding tool), 45

\section{S}

Sèt screw (transducer-tool), 27,48

Sintering temperature of aluminum (bondability), 22

Sweep-frequency ultrasonic power supply, $50-54$

\section{T}

Temperature (power) cycling, $80-86$

Testing (bond pull test), 18,23-25,85-94

Tool (bonding)

composition, 65

design, 65

extension below transducer, $39,45,47$

polishing techniques, 21

resonance condition, 45

set screw, 27,48

studies of vibration modes, $39,41,45$, 65-7乎, 96

vibration amplitude, $11-14,65-73,94$ after changing tool, 45-48

Transducer

efficiency, 38

mount interaction, 41

$Q$ of, 43,50

temperature effects, 38

tool hole, 48-49

Trouble shooting the ultrasonic system, 26-28

Tune-up procedure (ultrasonic bonding machine), 26-28
U

Ultrasonic bond monitoring, 58 -73

Ultrasonic bonding mechanism, 73-80

Ultrasonic power supply, 13, 50-56 impedance, 50 phase locked loop, 55

sweep frequency, $50 .-54$

Ultrasonic system problems, 45-57

Units , 3

\section{v}

Vibration amplitude of bonding tools, 11-14, $65-73,94,96$

Vibration amplitude variations after changing tool, 45-48

Vibration (extraneous) effect on bonding, $29-36$

Vibration nodes of bonding tools, measurement, 14

\section{W}

Wave form

bond monitoring, $63,65,67,69,71,72$

sweep frequency supply, 52, 54

transducer mount interaction, 42

Wire (aluminum), $19,90,94-.95$

Wire bond failure due to power cycling, $80-86$

Wire bond flexure, $80-86$

Wire bond loop, 80 -93

Wire clamp problems, 36

Wire elongation, $90-92$

Wire (gold), $94-.95$

Wire indentation tester, 19

wire (large diameter), $90-93$ 
CORM NBS-114A $(1.71)$
U.S. DEPT. OF COMM.
BIBLIOGRAPHIC DATA
SHEET

4. TITLE AND SUBTITLE

Microelectronic Ultrasonic Bonding

(Selected work from the NBS Microelectronic Bonding Project)

\section{AUTHOR(S)}

George G. Harman, Editor

9. PERFORMING ORGANIZATION NAME AND ADDRESS

NATIONAL BUREAU OF STANDARDS

DEPARTMENT OF COMMERCE

WASHINGTON, D.C. 20234

12. Sponsoring Organization Name and Address

National Bureau of Standards, Defense Nuclear Agency, U.S. Navy Strategic Systems Project Office, National Aeronautics and Space Administration/Marshall Space Flight Center

Gov' Accession
3. Recipient's Accession No.

5. Publication Date

January 1974

6. Performing Organization Code

8. Performing Organization

10. Project/Task/Work Unit No.

11. Contract/Grant No.

13. Type of Report \& Period Covered

Fina 1

14. Sponsoring Agency Code

$\sqrt{15 . \text { SUPPLEMENTARY NOTES }}$

16. ABSTRACT (A 200-word or less factual summary of most significant information. If document includes a significant bibliography or literature survey, mention it here.)

This report primarily comprises excerpts of the work done under the NBS ultrasonic wire bonding program that was previously published in 17 quarterly and other reports. The material is organized into subject groupings with the intention of presenting in convenient form sufficient information for making high quality ultrasonic wire bonds as well as imparting a basic understanding of the ultrasonic systems used. The work emphasizes problems and methods of solving them. To accomplish this, the required measurement equipment is first introduced. This is followed by procedures and techniques used in setting up a bonding machine, and then various machine-or operatorinduced reliability problems are discussed. The characterization of the ultrasonic system and its problems are followed by in-process bonding studies and work on the ultrasonic bonding (welding) mechanism. The report concludes with a discussion of various effects of bond geometry and wire metallurgical characteristics. Where appropriate, the latest, most accurate value of a particular measurement has been substituted for an earlier reported one. Thus all of the included material is up to date.

17. KEY WORDS (Alphabetical order, separated by semicolons) Bonding; degradation (wire bond); discrete devices; electrical interconnection; fabrication (wire bond); failure (wire bond); hybrid circuits; integrated circuits; microelectronics; reliability; semiconductor devices; 18. AVAILABILITY STATEMENT testing (wire bond); uitrasonic 19. SECURITY CLASS bonding; wire bond.

X] UNL IMIT ED. (THIS REPORT) 21. NO. OF PAGES FOR OFFICIAL DIST RIBUTION. DO NOT RELEASE TO NTIS.

\begin{tabular}{|l|} 
UNCL ASSIFIED \\
\hline 20. SECURITY CLASS \\
(THIS PAGE) \\
UNCL ASSIFIED \\
\hline
\end{tabular}

109

22. Price

$\$ 1.25$ 




\section{NBS TECHNICAL PUBLICATIONS}

PERIODICALS

JOURNAL OF RESEARCH reports National Bureau of Standards research and development in physics, mathematics, and chemistry. Comprehensive scientific papers give complete details of the work, including laboratory data, experimental procedures, and theoretical and mathematical analyses. Illustrated with photographs, drawings, and charts. Includes listings of other NBS papers as issued.

Published in two sections, available separately:

\section{- Physics and Chemistry (Section A.)}

Papers of interest primarily to scientists working in these fields. This section covers a broad range of physical and chemical research, with major emphasis on standards of physical measurement, fundamental constants, and properties of matter. Issued six times a year. Annual subscription: Domestic, $\$ 17.00$; Foreign, $\$ 21.25$.

\section{- Mathematical Sciences (Section B)}

Studies and compilations designed mainly for the mathematician and theoretical plyysicist. Topics in mathematical statistics, theory of experiment design, numerical analysis, theoretical physics and chemistry, logical design and programming of computers and computer systems. Short numerical tables. Issued quarterly. Annual subscription: Domestic, $\$ 9.00$; Foreign, $\$ 11.25$.

\section{DIMENSIONS, NBS}

The best single source of information concerning the Bureau's measurement, research, developmental, cooperative, and publication activities, this monthly publication is designed for the layman and also for the industry-oriented individual whose daily work involves intimate contact with science and technology - for engineers, chemists, physicists, research managers, product-development managers, and company executives. Annual subscription: Domestic, \$6.50; Foreign, \$8.25.

\section{NOKPERIODICALS}

Applied Mathematics Series. Mathematical tables, manuals, and studies.

Building Science Series. Research results, test inethods, and performance criteria of building materials, components, systcms, and structures.

Handbooks. Recommended codes of engineering and industrial practice (including safety codes) developed in coopcration with interested industries, professional organizations, and regulatory bodies.

Special Publications. Proceedings of NBS conferences, bibliographies, annual reports, wall charts, pamphlets, etc.

Monographs. Major contributions to the technical literature on various subjects related to the Bureau's scientific and technical activities.

National Standard Reference Data Series. NSRDS provides quantitative data on the physical and chemical properties of materials, compiled from the world's literature and critically evaluated.

Product Standards. Provide requirements for sizes, types, quality, and methods for testing various industrial products. These standards are developed cooperatively with interested Government and industry groups and provide the basis for common understanding of product characteristics for both buyers and scllèrs. Their use is voluntary.

Technical Notes. This series consists of communications and reports covering both other-agency and NBS-sponsored work) of limited or transitory interest.

Federal Information Processing Standards Publications. This serics is the official publication within the Federal Government for information on standards adopted and promulgated under the Public Law 89-306, and Bureau of the Budget Circular A-86 entitled, Standardization of Data Elements and Codes in Data Systems.

Consumer Information Series. Practical information. based on NBS research and experience, covering arcas of interest to the consumer. Easily understandable language and illustrations provide useful background knowledge for shopping in today's technological marketplace.

\section{BIBLIOGRAPHIC SUBSCRIPTION SERVICES}

The following current-awareness and literature-survey bibliographies are issued periodically by the Bureau:

Cryogenic Data Center Current Awareness Service (Publications and Reports of Interest in Cryogenics). A literature survey issued weekly. Annual subscription: Domestic, $\$ 20.00$; foreign, $\$ 25.00$.

Liquefied Natural Gas. A literature survey issued quarterly. Annual subscription: $\$ 20.00$.

Superconducting Devices and Materials. A literature survey issued quarterly. Annual subscription: $\$ 20.00$. Send subscription orders and remittances for the preceding bibliographic services to the U.S. Department of Commerce, National Technical Information Service, Springfield, Va. 22151.

Electromagnetic Metrology Current Awareness Service (Abstracts of Selected Articles on Measurement Techniques and Standards of Electromagnetic Quantities from D-C to Millimcter-IVave Frequencies). Issued monthly. Annual subscription: $\$ 100.00$ (Special rates for multi-subscriptions). Send subscription order and remittance to the Electromagnetic Metrology Information Center, Electromagnetics Division, National Bureau of Standards, Boulder, Colo. 80302.

Order NBS publications (except Bibliographic Subscription Services) from: Supcrintendent of Documcnts, Government Printing Office, Washington, D.C. 20402. 
U.S. DEPARTMENT OF COMMERCE National Bureau of Standards

Washington, D.C. 20234

POSTAGE AND FEES PAID U.S. DEPARTMENT OF COMMEACE $C O M-215$
OFFICIAL BUSINESS

Penalty for Private Use, $\$ 300$

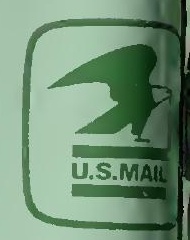

